I NTER NATIONAL MONETARY FUND

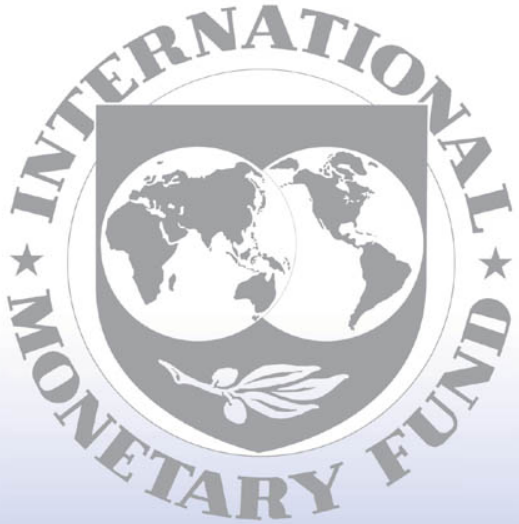

Staff

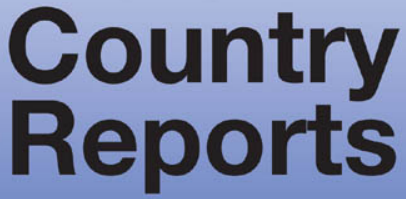




\title{
Uruguay: Third Review Under the Stand-By Arrangement and Request for Modification and Waiver of Nonobservance of Performance Criteria-Staff Report; Staff Statement; Press Release on the Executive Board Discussion; and Statement by the Executive Director for Uruguay
}

In the context of the third review under the Stand-By Arrangement and request for a modification and a waiver of nonobservance of performance criteria, the following documents have been released and are included in this package:

- the staff report for the Third Review Under the Stand-By Arrangement and Request for Modification and Waiver of Nonobservance of Performance Criteria, prepared by a staff team of the IMF, following discussions that ended on February 23, 2006, with the officials of Uruguay on economic developments and policies. Based on information available at the time of these discussions, the staff report was completed on March 14, 2006. The views expressed in the staff report are those of the staff team and do not necessarily reflect the views of the Executive Board of the IMF.

- a staff statement of March 24, 2006 updating information on recent developments.

- a Press Release summarizing the views of the Executive Board as expressed during its March 27, 2006 discussion of the staff report that completed the review.

- a statement by the Executive Director for Uruguay.

The documents listed below have been or will be separately released.

Letter of Intent sent to the IMF by the authorities of Uruguay*

Memorandum of Economic and Financial Policies by the authorities of Uruguay*

Technical Memorandum of Understanding by the authorities of Uruguay*

*Also included in the Staff Report

The policy of publication of staff reports and other documents allows for the deletion of market-sensitive information.

To assist the IMF in evaluating the publication policy, reader comments are invited and may be sent by e-mail to publicationpolicy@imf.org.

Copies of this report are available to the public from

International Monetary Fund • Publication Services

$70019^{\text {th }}$ Street, N.W. • Washington, D.C. 20431

Telephone: (202) 623-7430 • Telefax: (202) 623-7201

E-mail: publications@imf.org • Internet: http://www.imf.org

Price: $\$ 15.00$ a copy

\author{
International Monetary Fund \\ Washington, D.C.
}


This page intentionally left blank

CInternational Monetary Fund. Not for Redistribution 
INTERNATIONAL MONETARY FUND

URUGUAY

\title{
Third Review under the Stand-By Arrangement and Request for Modification and Waiver of Nonobservance of Performance Criteria
}

\author{
Prepared by the Western Hemisphere Department \\ (In collaboration with other Departments)
}

Approved by Ranjit Teja and Juha Kähkönen

March 14, 2006

Stand-By Arrangement. On June 8, 2005, a three-year SBA was approved under the exceptional access procedures (SDR 766.3 million, 250 percent of quota). SDR 674.3 million remain to be disbursed, and a purchase of SDR 85.8 million will become available upon completion of this (third) review. Outstanding Fund obligations as of end-2005 were SDR 1,609.3 million (526 percent of quota). At the second review, repurchase expectations arising between February and December 2006, and amounting to SDR 540.9 million, were extended to an obligations basis.

\section{Economic developments have been favorable:}

- Growth remains strong, and is likely to have exceeded the 2005 target of 6 percent.

- Fiscal results have been better than programmed, with a 2005 primary surplus of 3.8 percent of GDP compared to the 3.5 percent target.

- Inflation has edged up to 6.6 percent, but is still within the central bank's target range.

- International reserves have strengthened further reflecting significant capital inflows.

- The banking system reacted calmly to the recent intervention of the credit cooperative COFAC.

The program is on track. All performance criteria for this review are estimated to have been met, except for the submission of tax reform, which is expected to be met with a short delay (prior action for this review) and for which a waiver is requested. Final data will be reported prior to the Board meeting.

Review issues. Policy discussions centered on the macroeconomic framework and the structural reform agenda for 2006, in particular tax, financial sector, and pro-growth reforms. A $1 / 4$ percent of GDP fiscal adjustor on the use of privatization revenue for infrastructure investment is proposed. The authorities also request that the June performance criterion on the approval of tax reform be deferred to October, on account of the already crowded legislative agenda. However, the new tax system will become effective in January 2007 as previously envisaged.

Fund relations. The last Article IV consultation was concluded on July 11, 2003, and an Ex Post Assessment was considered on March 18, 2005. The next Article IV consultation is planned for mid2006.

Discussions. A mission visited Montevideo during February 10-23 and met with Finance Minister Astori, Central Bank President Cancela, other senior officials, and private sector representatives. The team comprised M. Piñón (Head), J. Canales-Kriljenko, S. Eble, (all WHD), A. Hajdenberg (FAD), H. Finger (PDR), and G. Gelos (Resident Representative). R. Teja (WHD) and D. Vogel (OED) participated in some of the policy discussions. 


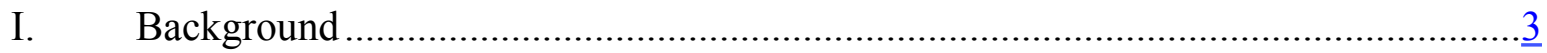

II. Macroeconomic Outlook and Risks...............................................................

III. Policy Discussions ........................................................................................

A. Fiscal and Debt Management Policy ……………………………………...

B. Monetary and Exchange Rate Policy ............................................................ 7

C. Financial Sector Reforms ..................................................................

D. Growth Agenda ............................................................................

VI. Program Financing and Monitoring.............................................................

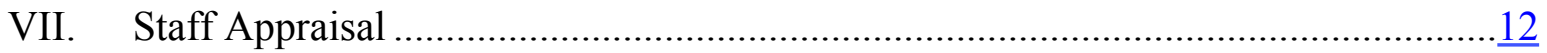

\section{Boxes}

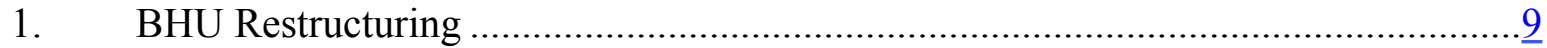

2. The Government's Growth Reform Agenda........................................................

Figures

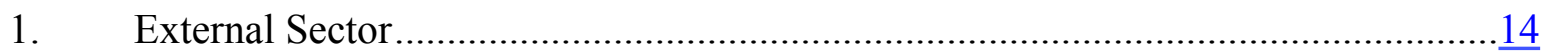

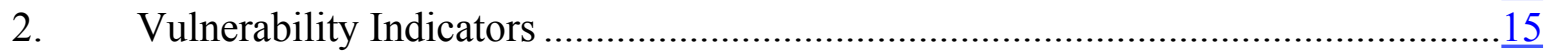

Tables

1. Selected Economic and Social Indicators.......................................................16

2. Performance under the 2005-06 Economic Program..............................................

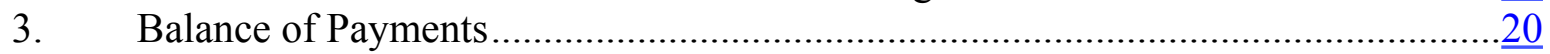

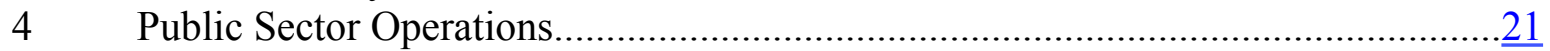

5. Summary Accounts of the Banking System .......................................................

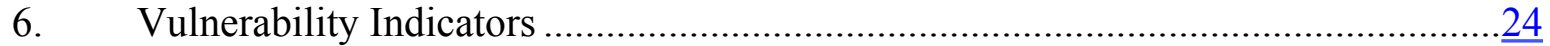

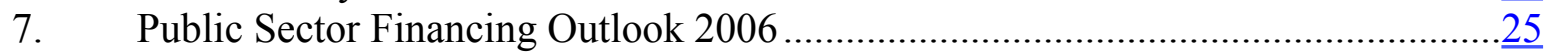

8. Schedule of Purchases Under the Stand-By Arrangement, 2005-08 …...................

9. Projected Payments to the Fund and Capacity to Repay ...........................................27

Appendices

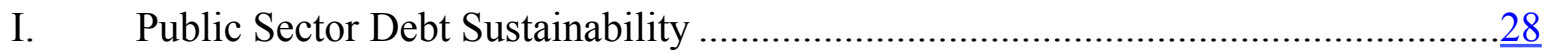

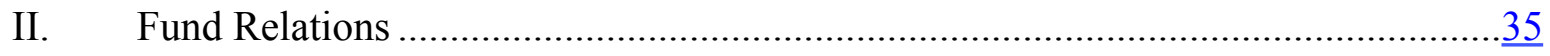

III. Relations with the World Bank Group...............................................................

IV. Relations with the Inter-American Development Bank ........................................... 40

Attachments

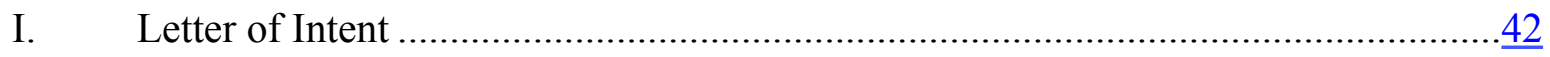

II. Supplementary Memorandum of Economic and Financial Policies..........................43

III. Technical Memorandum of Understanding ………………..................................50 


\section{BACKGROUND}

1. The program is on track and economic developments remain favorable:

- Growth continues to be robust. Driven mainly by a pick up in consumption, GDP growth for 2005 is likely to have exceeded the target of 6 percent, with real GDP surpassing its pre-recession peak level of 1998. For 2006, growth is envisaged to reach at least 4 percent. Industrial production continues to display strength, and export growth, although moderating remains high. While employment has risen, unemployment has remained stable at 12 percent, reflecting a continued recovery in the labor participation rate.

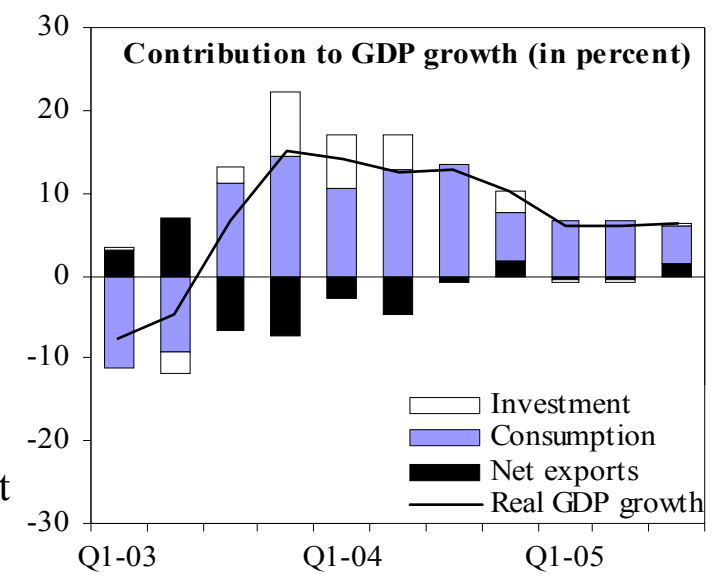

- $\quad$ Inflation has picked up to 6.6 percent within the central bank target range. End2005 inflation was 4.9 percent, well below the target range of $51 / 2-71 / 2$ percent, owing in part to the appreciation of the exchange rate. With the exchange rate stabilizing, water and electricity tariff adjustments, salary adjustments in early 2006, and a pick up in monetary growth, 12-month inflation edged up to 6.6 percent in February. The central bank inflation target range continues at 5-7 percent for March 2006 and

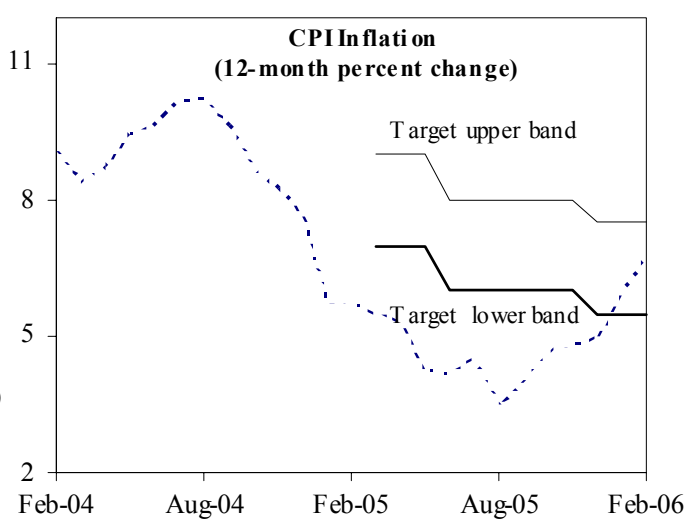
$4 \frac{1}{2}-6 \frac{1}{2}$ percent for end-2006.

- $\quad$ Fiscal results have been better than programmed. The primary balance in 2005 reached 3.8 percent of GDP, compared to a target of 3.5 percent, mainly reflecting buoyant tax revenues, which were partly offset by weaker than expected public enterprise performance. Overall, outlays were broadly as programmed.

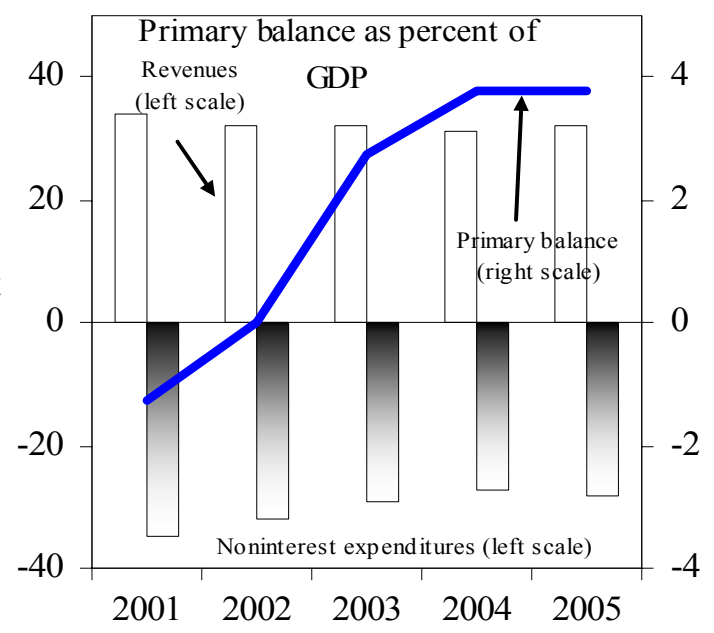


2. The authorities have taken advantage of favorable market conditions to reduce vulnerabilities further. With sovereign spreads near historical lows, the ministry of finance placed US\$500 million in January, thus completing a substantial portion of the total international market issuance foreseen in the program baseline for 2006. The central bank also took advantage of strong capital inflows to purchase some US\$450 million between November and February 2006. In addition, there has been an improvement in the level and structure of public debt, with the debt ratio declining to 70 percent of GDP by end-2005 in the wake of fiscal consolidation, solid growth, and a more appreciated currency; the average maturity of debt too has lengthened from 6 years at end-2004 to $7 \frac{1}{2}$ years at end-2005. Nevertheless, medium-term financing requirements, averaging $8 \frac{1}{2}$ percent of GDP in 2007-08, remain high.

\section{With remaining weaknesses in the financial system being addressed, markets} reacted calmly to the recent intervention of COFAC. Following the intervention of the small credit cooperative COFAC, no spillovers to other banks were observed. Insured deposits recently have begun to be paid. Indicators of the overall health of the banking system show steady improvement and public bank restructuring continues.

- A detailed implementation plan for the restructuring of the housing bank BHU was adopted (structural benchmark for end-February).

- $\quad$ The sale of Nuevo Banco Comercial (NBC) is expected to be completed by April (structural benchmark for endJune).
Uruguay. Banking System Indicators: 2002-2005

\begin{tabular}{lcccc}
\hline & 2002 & 2003 & 2004 & 2005 \\
\hline Capital adequacy ratio & & & & \\
Banking system 1/ & -9.6 & 9.2 & 10.9 & 11.2 \\
BROU & 5.5 & 6.4 & 8.3 & 8.5 \\
Private banks & -27.4 & 11.3 & 13.0 & 12.9 \\
& & & & \\
Return on assets & -25.6 & -1.1 & -0.1 & 0.7 \\
Banking system 1/ & -17.5 & 0.1 & 0.5 & 0.6 \\
BROU & -35.2 & -2.0 & -0.2 & 1.0 \\
Private banks & & & & \\
Nonperforming loans & 31.5 & 8.6 & 3.8 & 2.5 \\
Banking system 1/ & 33.1 & 10.2 & 2.6 & 2.4 \\
BROU & 31.4 & 6.4 & 3.6 & 1.6 \\
Private banks & & & & \\
& & & & \\
\hline Source: Superintendency of Banks and Fund staffestimates. &
\end{tabular}

Source: Superintendency of Banks and Fund staff estimates.

1/ Excludes the housing bank BHU, which no longer conducts financial intermediation.

- $\quad$ The draft law on the financial sector reform is currently being discussed in the Senate.

4. The implementation of the rest of the structural agenda is also broadly on track. The available data suggest that all quantitative end-December performance criteria were met, while those for end-March are well within reach. 
- $\quad$ The submission of the tax reform bill to congress (performance criterion for endFebruary) has been slightly delayed but is expected to take place shortly (prior action for the Board meeting).

- $\quad$ The growth commission is about to publish a set of proposals to foster investment and growth (structural benchmark for end-March).

- $\quad$ A five-year spending plan was enacted in December 2005 (structural benchmark for end-February) in the context of the adoption of the 2005-09 budget.

- $\quad$ Progress is also being made in other areas of the structural agenda for 2006, including pension, revenue administration, bankruptcy, and budget reforms.

- $\quad$ Final data on all performance criteria will be provided ahead of the Board meeting.

5. Some political risks have emerged, including a dispute with Argentina and some environmental groups that has stalled the cross-border road traffic. Protesting against the planned pulp mill projects on the river Uruguay, activists have been blocking the bridges connecting Argentina and Uruguay, disrupting the flow of goods and people. While the impact on the recent summer tourism season has been moderate, the economic effect of a sustained closure of the bridges could be significant.

\section{MACROECONOMIC OUTLOOK AND RISKS}

6. The outlook for $2006-2008$ and medium-term vulnerabilities remain broadly unchanged from the last review. Although continued rise in leading indicators suggest upside potential for growth, the recent bridge blockings on the Argentine border pose some risks. Accordingly the growth projection for 2006 has been left at 4 percent. End-2006 inflation is expected to be 5.5 percent. Projected international reserves have been revised upwards as a result of the stronger end-2005 outturn and continued capital inflows. High public debt, the dollarized financial system, and pressures for public expenditure remain important vulnerabilities.

\begin{tabular}{|c|c|c|c|c|c|c|c|c|}
\hline \multicolumn{9}{|c|}{$\begin{array}{l}\text { Uruguay: Medium-term Macroeconomic Framework } \\
\text { (Percent of GDP, unless otherwise indicated) }\end{array}$} \\
\hline & \multirow[b]{2}{*}{2003} & \multirow[b]{2}{*}{2004} & \multicolumn{2}{|l|}{2005} & \multicolumn{2}{|l|}{2006} & \multicolumn{2}{|c|}{ Proj. } \\
\hline & & & Report $06 / 123$ & $\overline{\text { Est. }}$ & Report $06 / 123$ & Proj. & 2007 & 2008 \\
\hline Real GDP (percentage change) & 2.2 & 12.3 & 6.0 & 6.0 & 4.0 & 4.0 & 3.5 & 3.0 \\
\hline CPI (eop, percentage change) & 10.2 & 7.6 & 5.9 & 4.9 & 5.5 & 5.5 & 4.7 & 4.4 \\
\hline Revenue & 32.0 & 30.9 & 32.0 & 32.2 & 33.0 & 33.0 & 33.5 & 33.6 \\
\hline Non-interest expenditure & 29.3 & 27.2 & 28.4 & 28.4 & 29.3 & 29.3 & 29.5 & 29.6 \\
\hline Primary balance & 2.7 & 3.8 & 3.6 & 3.8 & 3.7 & 3.7 & 4.0 & 4.0 \\
\hline Overall fiscal balance & -3.2 & -2.2 & -1.2 & -0.8 & -1.1 & -1.1 & -0.6 & -0.5 \\
\hline Public sector debt & 104 & 92 & 73 & 70 & 69 & 65 & 62 & 59 \\
\hline External current account balance & -0.5 & -0.7 & -2.4 & -2.0 & -5.8 & -5.6 & -3.6 & -3.1 \\
\hline excluding: pulp mill projects & -0.5 & -0.7 & -2.1 & -1.5 & -2.1 & -2.1 & -2.2 & -1.9 \\
\hline Official reserves (millions of U.S. dollars) & 2,087 & 2,512 & 3,110 & 3,438 & 3,255 & 3,865 & 4,015 & 4,165 \\
\hline As a $\%$ of ST debt + FX deposits & 20.0 & 27.7 & 29.7 & 33.1 & 28.8 & 34.4 & 37.2 & 38.5 \\
\hline
\end{tabular}




\section{Policy Discussions}

7. In addition to the review of macroeconomic policies, discussions centered on key structural reforms for this year. The main focus was on tax reform, the restructuring plan for the state housing bank BHU, the situation of the credit cooperative COFAC, and on measures to enhance the business environment and promote investment.

\section{A. Fiscal and Debt Management Policy}

\section{Revenue administration reforms are progressing well, and the authorities remain} confident that their ambitious revenue targets for this year will be met. With administration efforts already yielding significant revenues (the social security agency and the tax directorate exceeded their targets for 2005), the reform plan for the tax directorate progressing well, and the plan to restructure customs expected to be ready around midyear (structural benchmark for end-August), the authorities expect to achieve their revenue collection targets for 2006. Public enterprises are expected to improve their performance significantly in 2006, following weather-related electricity generation cost overruns and the rapid pace of the international oil price increases in 2005, which temporarily resulted in tariff adjustment lags. Staff encouraged the authorities to devote adequate efforts and resources to ensure the tax reform is effective by 2007 , in particular the administration of the new personal income tax.

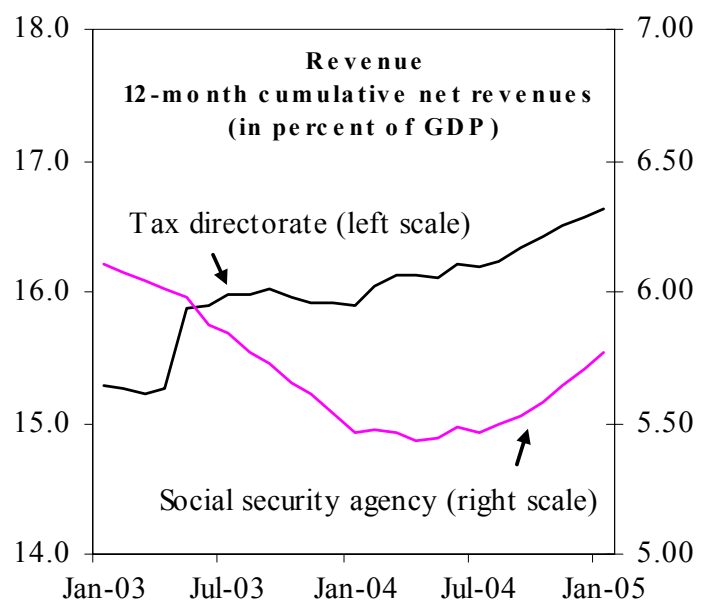

9. An adjustor to the 2006 fiscal targets of up to US\$50 million ( $1 / 4$ percent of GDP) is proposed to accommodate higher investment financed with privatization proceeds. To ease infrastructure bottlenecks to growth over the medium term, the authorities plan to undertake a few high-quality investment projects during July 2006-June 2007, with high financial and economic returns, in which the public sector would act as a catalyst to attract private participation. In this context, the authorities are planning to invest, in association with the private sector, up to US\$25 million in railroad infrastructure to support the increased freight transportation demand derived from the new pulp/forestry projects; and about US\$14 million in a dredging project in the port of Montevideo. Officials underlined that these and other potential projects would be subjected to stringent evaluation and monitoring standards. The authorities indicated that they do not expect to offer government guarantees, that potential risks would be appropriately shared with the private sector, and that all transactions, including any

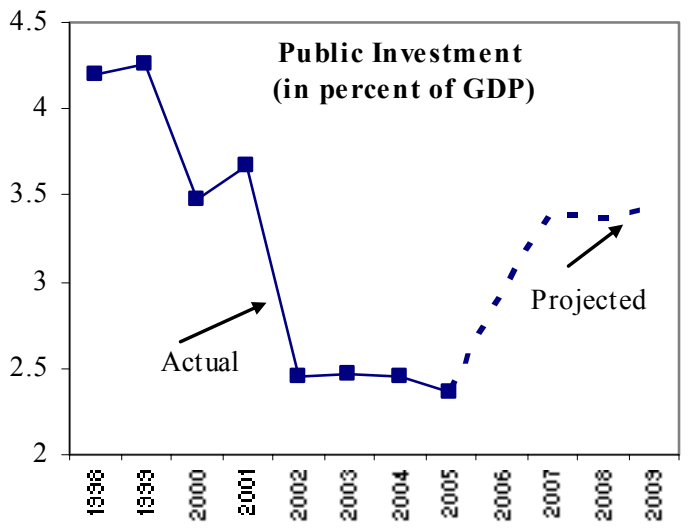


guarantees, would be transparently recorded in the fiscal accounts. The authorities reiterated that a primary surplus of 3.7 percent of GDP for 2006 and 4.0 percent for 2007 remained their goals, but regarded the adjustor as a safety valve to allow them to undertake these projects. They remain committed to saving any revenue overperformance to reach the 4 percent mediumterm target as soon as possible.

10. The draft tax reform package is expected to be submitted to congress in March, slightly later than envisaged under the end-February performance criterion. The authorities stated that the draft law would be broadly in line with the proposal made public earlier (see Country Report No. 06/123). Main revisions would include an additional personal income tax bracket (estimated to be revenue neutral) and limited deductions for health expenditures. To protect the fiscal objectives, the draft would envisage a smaller reduction in the top VAT rate (to 22 percent instead of 21 percent) until the revenue targets are achieved. The authorities explained that these modifications addressed the key comments received in the public consultation on the proposal. The authorities explained that, in light of a very full agenda in congress, they now viewed it as difficult that the law could be approved by June 2006, as envisaged. For this reason, they requested a modification of the performance criterion, changing the beginning of the implementation to end-October 2006. Lest the delay in congressional approval postpone the effective date in 2007, the authorities will work in parallel on the implementing regulations so that the law can still go into effect as planned next January.

\section{The new debt office has begun operations, with key objectives including a} reduction in dollar debt and a smoothing of the repayment profile. Officials explained that the unit had started to build a debt database and had been finalizing the financing plan for the government. The office already took the lead in successfully completing the recent issuance of US\$500 million on international capital markets. A debt strategy is being prepared with Fund technical assistance, aiming at developing the local capital market, increasing the share of peso and inflation-indexed debt, lengthening maturities, and smoothing the amortization profile.

\section{B. Monetary and Exchange Rate Policy}

\section{The authorities viewed recent exchange-rate} stability as temporary. The nominal exchange rate visà-vis the U.S. dollar had remained flat for over a month as a result of foreign exchange purchases by the authorities, including as an indirect result of foreign exchange forward purchases by the Treasury to cover future debt service (The central bank, as the residual supplier in the forward market, hedges the higher forward exposure by purchasing equivalent amounts of foreign exchange in the spot market). Staff urged the authorities to allow the exchange rate to better reflect market conditions, highlighting the dangers of signaling a floor for the exchange rate and the difficulties in pursuing multiple objectives with one instrument. Officials stressed that the intervention had been

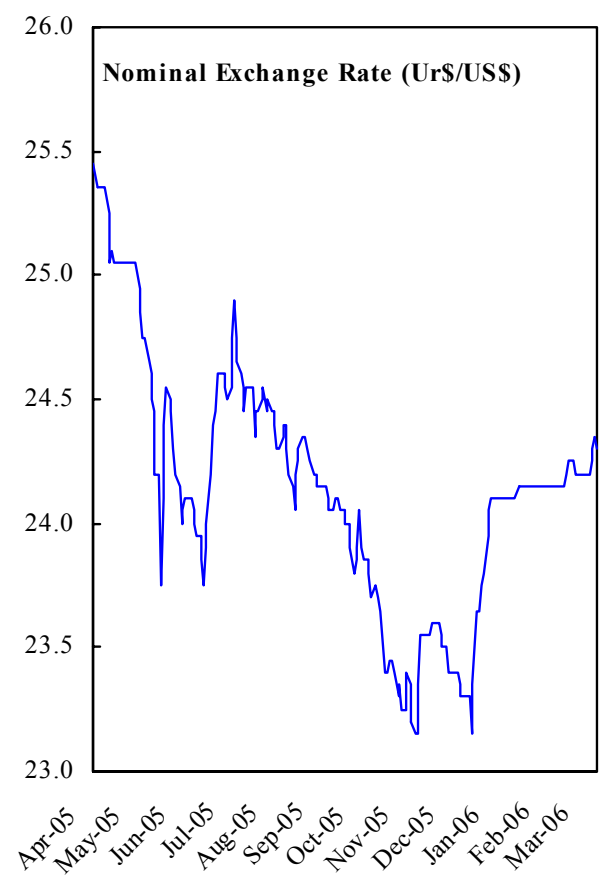

CInternational Monetary Fund. Not for Redistribution 
opportunistic, to build gross reserves, and necessary to break a cycle of self-reinforcing appreciation expectations, but that this did not imply a fundamental change to monetary and exchange rate policy. They pointed out that the exchange rate had started to regain flexibility and explained that, while there was some concern about excessive appreciation, inflation remained the overriding objective of monetary policy.

13. To increase the focus on the ultimate inflation objective, the central bank has begun to deemphasize base money targets in its public statements. The authorities are now announcing the annual growth rate of their intermediate target (M1) instead of base money target ranges for the four quarters ahead, while maintaining base money as their internal operational variable. The authorities explained that the change in the communication strategy intends to convey that their commitment is with the inflation target, and that the increased flexibility in money management would allow them to gradually move in the direction of a fully-fledged inflation targeting framework. The authorities agreed with staff that such a move would require further analytical work on inflation forecasting and channels of monetary policy influence on prices.

\section{The authorities explained that the strong increase in monetary aggregates toward} the end of last year was necessary to satisfy money demand. They stressed that inflation is well within their target range and the recent pick-up in part explained by temporary factors, including the timing of tariff and salary adjustments. They acknowledged risks, however, due to uncertainty about the evolution of money demand. They also pointed out that inflation expectations for 2006 were within the target range. Nevertheless, staff and the authorities agreed that inflationary risks had increased and that pressures needed to be monitored carefully over the next months. Against that background, the authorities reaffirmed their commitment to tighten policies as necessary to meet the inflation targets and decided that, for now, it would be more prudent not to accommodate last year's overrun in M0 and to maintain the agreed targets for 2006, implying a substantial slowdown in money growth in the remainder of 2006. The appropriateness of this stance will be reassessed at the next review.

\section{Financial Sector Reforms}

\section{The authorities have adopted a detailed implementation plan for reforming the} housing bank BHU. The plan aims to transform BHU into a residential mortgage lender that operates on a commercial basis and ceases social activities, which will be transferred to the Ministry of Housing (Box 1). The authorities committed to transfer nonperforming loans to a fiduciary trust and to recapitalize BHU by end-August (new performance criterion), a necessary measure for the bank to have a clean balance sheet. By November, remaining problem assets will be transferred, regulatory measures put in place to refocus BHU business solely on residential mortgage lending on a commercial basis, and BHU will be required to meet all prudential standards and submit a viable business plan (new performance criterion).

16. The intervention of COFAC highlighted the strengthening of Uruguay's financial system and its oversight. The authorities stressed that, for the first time in recent Uruguayan history, a bank intervention occurred (i) before a liquidity problem became a solvency problem; (ii) with the majority of depositors fully protected within a deposit insurance scheme; and (iii) 
without the use of government funds (other than through the previously envisaged US\$60 million for capital and loans to the deposit insurance agency, to be recovered through deposit insurance premia and asset recovery). It was agreed that any newly created institution building on COFAC's assets would have to be well-capitalized and provide a viable business plan. In this regard, the authorities noted that discussions with the Venezuelan Development Bank (Bandes) for a purchase and assumption operation were underway.

\section{Box 1. BHU Restructuring}

In February 2006, the authorities adopted a detailed implementation schedule for the restructuring of the state mortgage bank. This follows restructuring efforts during 2002-2004, which had included adopting a modified charter to turn BHU into a nonbank mortgage lender, with deposit taking ability limited to mortgage pre-saving schemes, transferring part of the nonperforming loan portfolio (US\$102 million book value) to a fiduciary trust fund whose beneficiary was the Ministry of Finance, and reducing BHU staff and labor costs by about 50 percent. While progress was achieved, these efforts, however, did not fully stabilize the financial condition of the institution as the improvement on the collection on remaining nonperforming assets and operational efficiencies expected under the restructuring Plan launched in December 2003 did not materialize and BHU was not able to secure new financing through securitization. By end 2005, BHU was not complying with prudential regulations, including reserve and capital requirements. Nevertheless, as a result of the restructuring efforts, BHU does not appear to pose a systemic liquidity risk.

The new restructuring plan of BHU aims to address the shortcomings of the previous effort by focusing BHU's mandate on commercial activities, while transferring social housing policy to the Ministry of Housing. For consistency with its new mandate and to comply with all prudential regulations, the plan calls for:

- Transferring BHU's nonperforming assets to a fiduciary trust, with the mandate, incentives, and resources to maximize the net present value of its assets;

- Transferring to the Ministry of Finance BHU's liabilities to BROU and the public sector in the amount needed to capitalize and restructure BHU, and with a view to reducing currency and maturity mismatches; and

- Adopting a business plan satisfactory to the Bank Superintendency that demonstrates BHU's ability to generate positive cash flows and profits going forward.

The business plan will include measures to further reduce BHU's overhead and other costs in line with the reduced size of its balance sheet, and to improve BHU's managerial structure, corporate governance, and internal control systems. BHU will refrain from lending until the Bank Superintendency considers that BHU has the systems in place to resume sound lending.

\section{Growth Agenda}

\section{The tripartite business environment commission is preparing to publish} recommendations to boost growth and investment (end-March structural benchmark). The work of the commission will become part of a presidential growth initiative. In various areas covered by the proposals, the government has already started to take specific actions, such as the tax reform and private-public associations to improve public infrastructure (Box 2). Similarly, a new draft law to prevent anticompetitive behavior has recently been sent to congress. On bankruptcy legislation reform, the authorities are holding another expert seminar 
to help inform the working group that is preparing a draft law, the submission of which is an end-June structural benchmark. Staff and the authorities agreed to discuss further specific measures during the next review.

\section{Box 2. The Government's Growth Reform Agenda}

A tripartite commission comprising government, labor and industry has been working on a comprehensive agenda to improve the business climate, increase investment, and improve resource allocation in the economy to lift up Uruguay's growth potential. Many of the commission's preliminary recommendations have already been incorporated in the government's reform agenda.

Key proposed reforms include:

- Increasing competition and strengthening the legal framework by amending bankruptcy procedures to include a Chapter 11-type regime (to be submitted to congress by June); strengthening the competition law (proposal currently with congress) and the legal framework for dispute resolution; and improving disclosure requirements of corporate financial information.

\begin{tabular}{llccccc}
\hline \multicolumn{7}{c}{ Uruguay: Assessment of the Environment for Business and Innovation } \\
\hline & Uruguay & Argentina & Brazil & Chile & $\begin{array}{c}\text { Costa } \\
\text { Rica }\end{array}$ \\
\hline Technology & $\mathbf{6 3}$ & 59 & 50 & 35 & 56 \\
Business Competitiveness Index & $\mathbf{7 0}$ & 64 & 49 & 29 & 50 \\
Quality of the National Business Environment & $\mathbf{6 6}$ & 64 & 52 & 29 & 53 \\
Financial market sophistication & $\mathbf{7 5}$ & 59 & 27 & 26 & 67 \\
Overall infrastructure quality & $\mathbf{5 0}$ & 59 & 85 & 32 & 93 \\
World Bank Business Indicator & $\mathbf{8 5}$ & 77 & 119 & 25 & 89 \\
& \multicolumn{7}{l}{} \\
Sources: Global Competitiveness Report 2005-2006, World Economic Forum (rank out of 117 countries); \\
World Bank Business Indicator (rank out of 155 countries). \\
\end{tabular}

- Increasing the efficiency of the tax system and reducing inequities: by eliminating exemptions, introducing a personal income tax, simplifying and reducing corporate income taxation, and eliminating low revenue yielding taxes. A proposal that includes these changes has been submitted to congress. In addition, ongoing improvements in revenue administration are reducing tax evasion.

- Public enterprise reforms: through improving governance, better linking wages to productivity, and transparent tariff setting, aiming to bring down the high cost of services provision.

- Simplifying business start-up procedures: by facilitating business registration and creating an investor relations office.

- Improving infrastructure: by strengthening the public investment framework (e.g., selection, execution, evaluation); establishing a sound framework for private participation in PPPs; and improving public expenditure management to make room for higher capital outlays in the budget.

\section{The authorities emphasized that labor-business relations were sound. Staff} expressed concern about reports on recent conflicts, which had led to the takeover of facilities by striking workers. The authorities explained that these had been isolated incidents, not representing a generalized trend and that legislation clarifying the rights and responsibilities of striking workers was being prepared. They also stressed that the tripartite wage councils had helped to stabilize labor relations, and that the authorities were keen on maintaining an environment that addressed genuine labor demands, while fostering an environment conducive to higher investment. 
19. The authorities highlighted that the implementation of the large pulp-mill projects was proceeding as planned. Once fully operational, the plants will generate about 1.3 percent of GDP in additional exports (see Country Report No. 06/123, Box 2). Staff inquired about possible negative effects of the ongoing conflict in light of the recent road blockings. The authorities explained that, while the actions had disrupted tourism somewhat, the season as a whole had been good. Moreover, they were confident that current difficulties can be resolved and that the implementation of the projects would proceed smoothly.

\section{Program FinanCing ANd Monitoring}

20. Close to half of the external bond placements assumed under the program baseline for 2006 have already been secured. In January 2006, the authorities issued a US\$500 million international bond under high demand and favorable conditions. The proceeds were used, in part, to advance amortization payments to the World Bank and IDB (including US\$237 million falling due in 2007). Nevertheless, large amortization payments from the 2003 debt exchange are falling due this year, and expected World Bank and IDB disbursements (US\$307 million) will require firm implementation of associated conditionality.

\section{Uruguay: 2006 Public Sector Financing}

(Nonfinancial public sector, in US\$ million)

\begin{tabular}{lcc}
\hline & Second Review & Rev. Proj. \\
\hline Amortization & $\mathbf{2 , 0 3 2}$ & $\mathbf{2 , 3 0 3}$ \\
Of which: World Bank/IDB & 505 & 741 \\
IMF & 630 & 678 \\
& & \\
Gross Financing & $\mathbf{2 , 3 9 9}$ & $\mathbf{2 , 6 2 3}$ \\
Multilaterals & & \\
Of which: World Bank/IDB & 432 & 307 \\
$\quad$ IMF & 543 & 543 \\
Market financing & 916 & 1,262 \\
Long-term bond financing & 400 & 400 \\
Of which: prefinancing for 2007 & 496 & 455 \\
UI/Letras & 12 & 56 \\
Other & & \\
\hline
\end{tabular}

Source: Fund staff estimates and projections.

21. Two new performance criteria on BHU restructuring, a modification on the performance criterion on tax reform implementation, and a new adjustor on public investment are proposed. All other quantitative performance criteria for 2006 remain as established at the time of the last review. Performance criteria, structural benchmarks, and indicative targets are specified in the supplementary MEFP and TMU (Attachments II and III). 
22. The authorities are committed to implementing the recommendations of the September 2005 safeguards assessment (Appendix II). Several recommendations have already been adopted, including incorporation of all Fund liabilities in the BCU's balance sheet and amending the criteria for selection and appointment of the external audit firm. With the implementation of the financial sector law, currently in congress, the key recommendations of the safeguards assessment would be in place.

\section{STAFF APPRAISAL}

23. Sound policies and a supportive external environment have sustained strong economic performance. The economy has grown at healthy rates and real wages have been recovering, and inflation, while it has increased, remains within the target range. Continued strong capital goods imports augur well for economic activity this year. These favorable results largely reflect the authorities' adherence to the macroeconomic policy commitments in their Fund-supported program.

24. Ensuring that growth is sustained into the medium term will require maintaining the momentum of the structural reform agenda. The government's intention to tackle the broader structural conditions for growth is very welcome, and the identification of key microeconomic and institutional reforms to boost investment and productivity by the growth commission is an important step. Transforming these recommendations into a concrete policy will be a focus of the next review, and decisive implementation thereafter will be essential.

25. Fiscal performance should remain strong if administrative reforms continue to be implemented forcefully. Revenues in both the social security agency and the tax directorate continue to exceed expectations, partly reflecting an increase in economic activity and a larger formal sector of the economy, but also improved tax administration. It is, however, critical to maintain a cautious execution of expenditures, adjusting public tariffs as needed, while moving ahead with the ongoing administrative reforms of the collection agencies so as to ensure smooth implementation of the tax reform and attainment of the ambitious revenue targets.

26. The draft tax reform will be a major step toward a more equitable and less distortionary tax system. The tax burden will be better distributed across the economy through the introduction of a personal income tax, the reduction of rates and of the number of exemptions, and a simplification of the system. The gradual reduction of indirect taxation adequately addresses the need to preserve revenue objectives. Staff supports the authorities request to move the date for congressional approval of tax reform from June to October, especially as steps are being taken to ensure that the January implementation date for the new tax regime is preserved.

27. The government's intention to attract private participation in public investment projects is welcome, but projects will need to be selected and monitored carefully. Staff supports the proposed adjustor to the fiscal targets given that, if well chosen, the investments will contribute to strengthen the foundation for medium-term growth and generate future fiscal revenues, as well as serve as models for future cooperation between the public and private 
sectors. The projects should not affect debt sustainability because they would be financed through privatization proceeds. It will, however, be key to ensure that the selected projects meet stringent quality criteria and minimize contingent fiscal risks.

28. Monetary policy has been successful, and needs to remain focused on achieving the inflation objective under a flexible exchange rate. Despite high oil prices and a strong economic expansion, inflation has remained subdued, which demonstrates the central bank's ability to conduct monetary policy in a new and complicated environment. Staff supports the authorities' strategy to deemphasize the role of base money targets in the context of an increased commitment to the inflation objective. Such a move, however, should be accompanied by a strengthened communication with the public on the central bank's inflation projections. While the continued accumulation of reserves is welcome, it will be important to maintain sufficient exchange flexibility, and avoid sending a signal to the market that there is a floor on the exchange rate. In light of the recent upticks in inflation, monetary policy will need to be vigilant to ensure that the inflation objective is achieved. In this regard, the decision to maintain the end-2006 indicative monetary targets, despite a higher-than-expected starting base is a welcome sign of prudence.

29. The current environment offers a unique opportunity to reduce further the still considerable risks. While vulnerabilities have been significantly lessened, debt levels remain high, reserves are still relatively low in light of the high dollarization of the economy, and substantial fiscal contingencies in the financial system remain. It is important that the government continues to use the opportunity provided by a strong economy and benign financial market conditions to accelerate debt reduction, improve the composition of the debt structure - particularly through a lengthening of maturities and increasing the share of domestic currency debt - and strengthening the financial sector.

30. Much has been achieved in the financial sector, but further restructuring of public banks is key to containing contingent fiscal liabilities. The prudent actions taken regarding COFAC and the announced payout of insured deposits helped maintain confidence in the banking system. Looking forward, approval of the financial sector reform package currently in congress will help to strengthen the financial system's resilience to shocks further. The government is taking the right steps to transform BHU into a viable mortgage lending institution, and strict adherence to the adopted implementation plan will be crucial to minimize fiscal risks.

31. In light of the strong performance under the program, and the positive outlook going forward, staff recommends completion of the third review. All performance criteria have been observed, except for the submission of the tax reform, which is expected to be completed as a prior action for the Board discussion; staff supports the associated waiver. 
Figure 1. Uruguay: External Sector
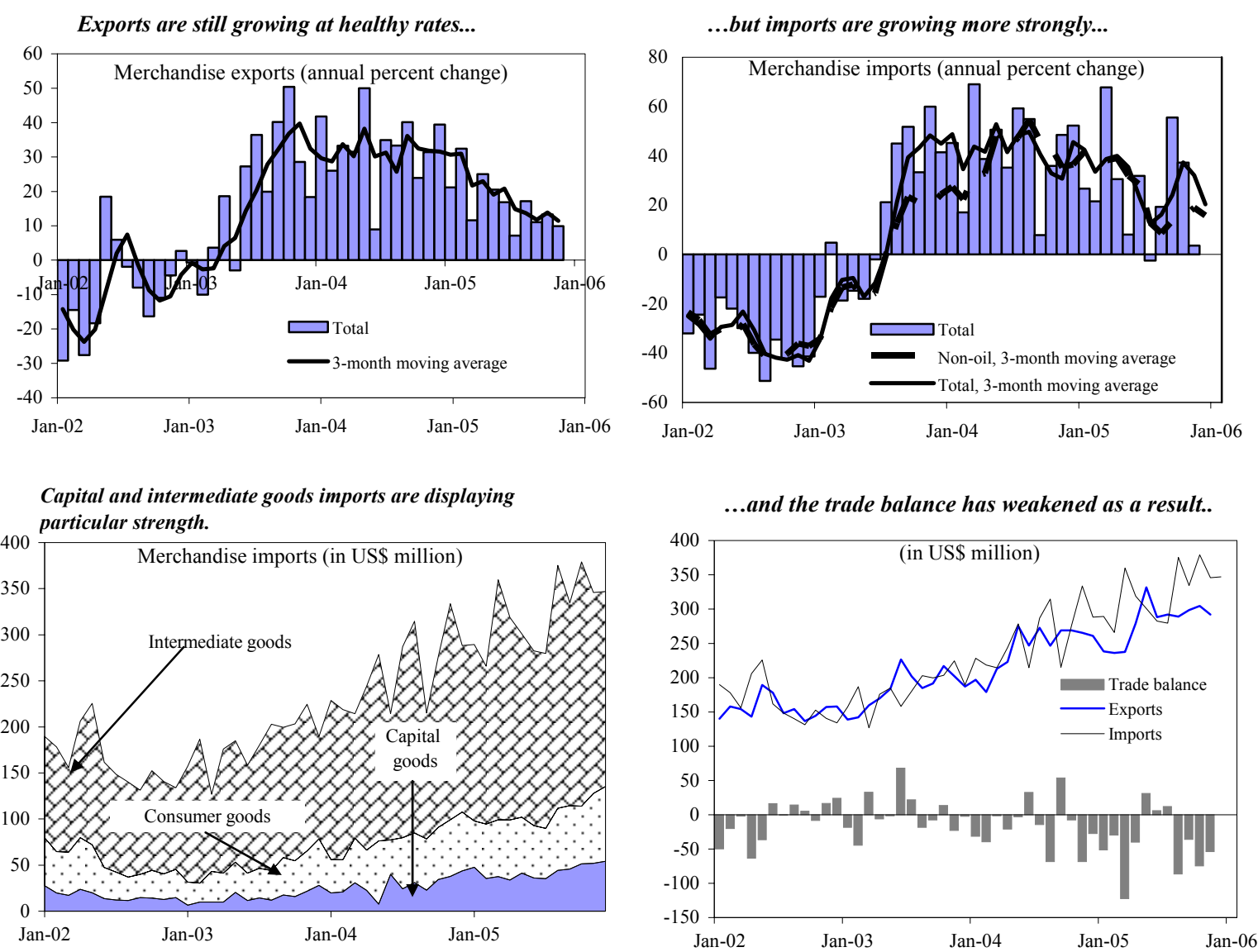

The financial account, however, has strengthened, and been helped by a rebound in portfolio investment.

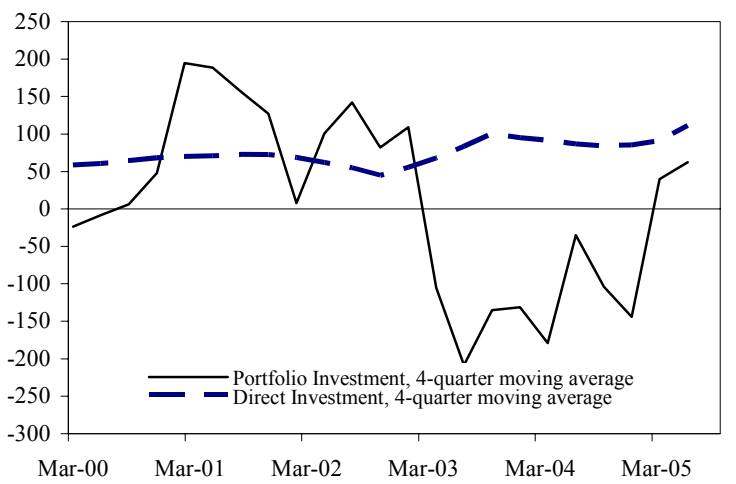

Despite the recent appreciation, the real exchange rate remains some 25 percent below pre-crisis levels.

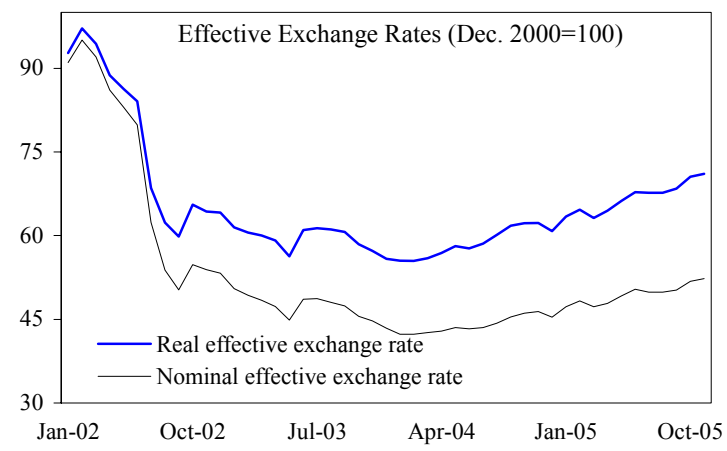

Sources: Central Bank of Uruguay; Ministry of Economy and Finance; and Fund staff estimates. 
Figure 2. Uruguay: Vulnerability Indicators

Public sector debt, while high, has been declining...
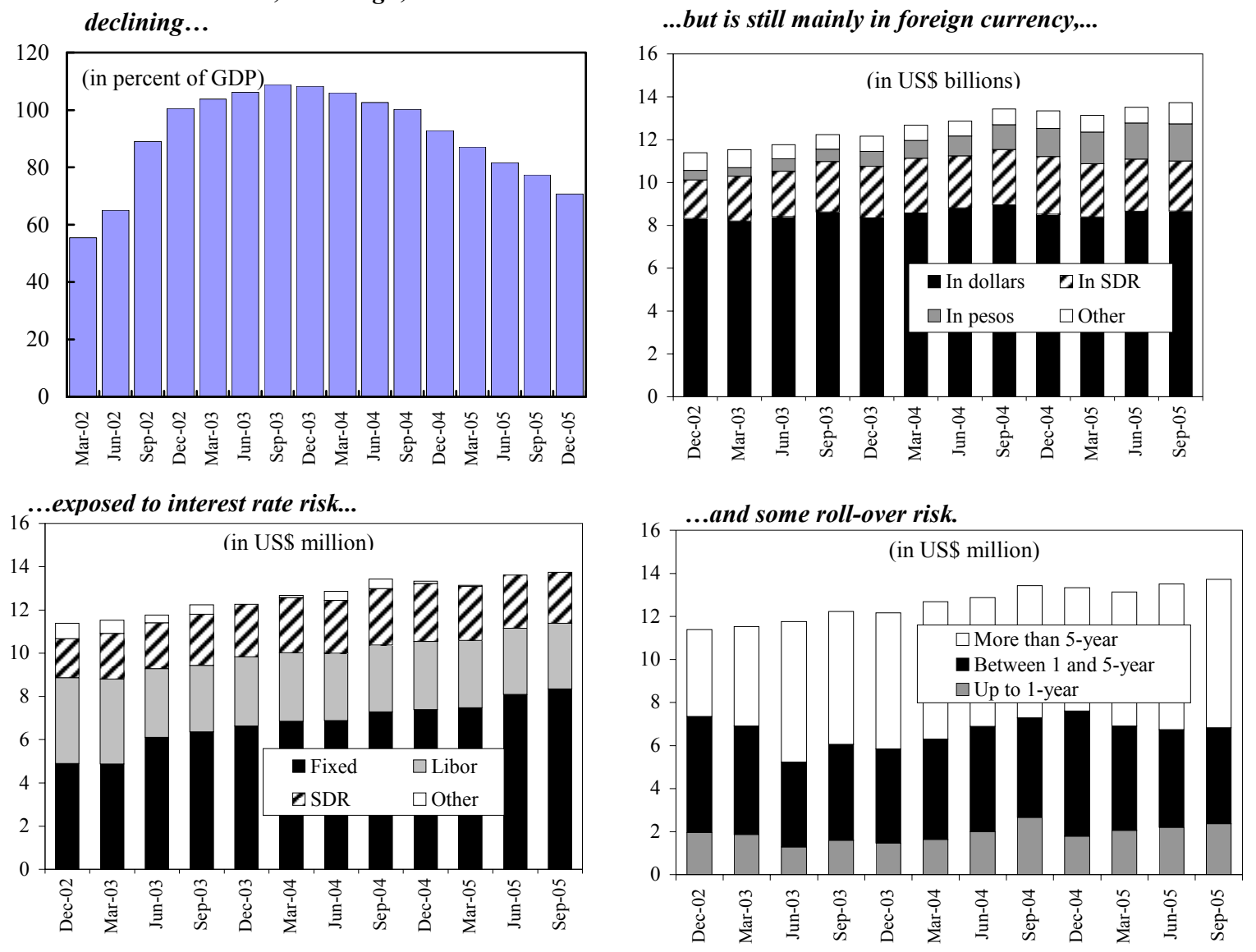

Dollarization in the financial system

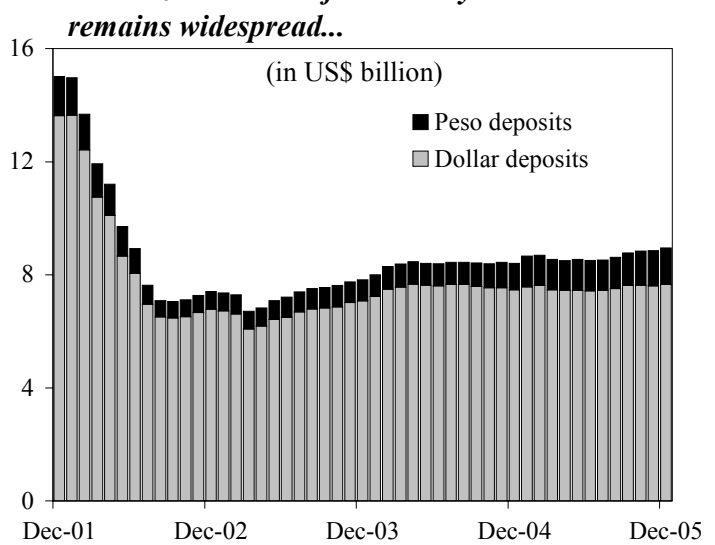

...and international reserves are still low for a dollarized economy.

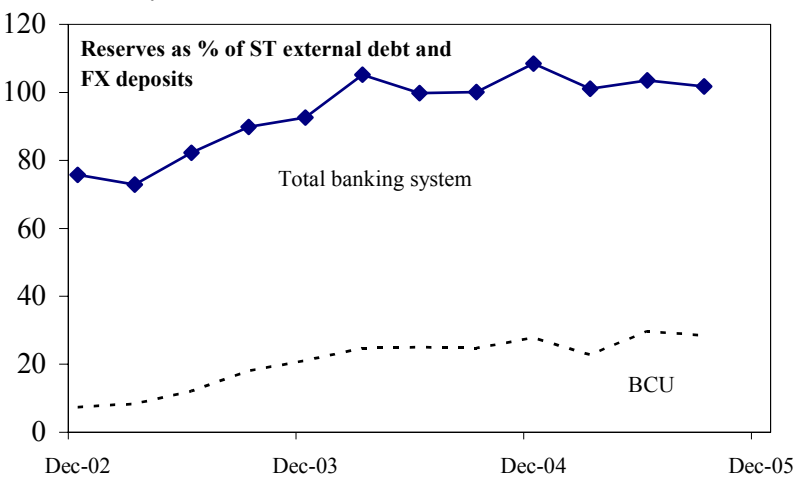

Sources: Central Bank of Uruguay; Ministry of Economy and Finance; and Fund staff estimates. 
Table 1. Uruguay: Selected Economic and Social Indicators

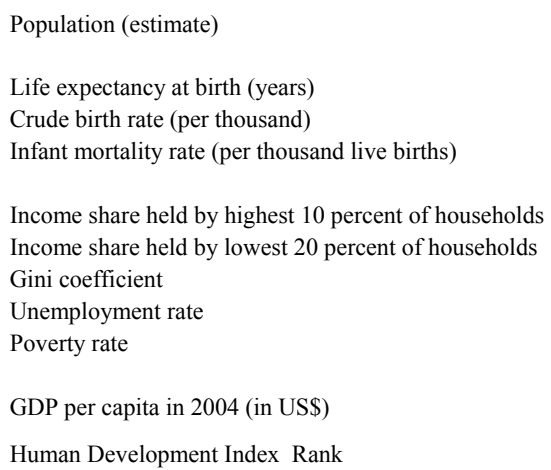

$\begin{array}{rlr}3.2 & \text { Physicians per 1,000 } & 3.7 \\ 74.6 & \text { Hospital beds per 1,000 } & 4.4 \\ 9.7 & \text { Access to safe water (percent of population) } & 98.0 \\ 14.0 & & \\ & & \\ 33.5 & \text { Adult literacy rate } & \\ 4.8 & \text { Gross enrollment rate } & 108.3 \\ 44.6 & \text { Primary education } & 101.5 \\ 11.0 & \text { Secondary education } & 37.7 \\ 31.0 & \text { Tertiary education } & \\ 4,300 & & \end{array}$

\begin{tabular}{|c|c|c|c|c|c|c|c|c|c|}
\hline & \multirow[b]{2}{*}{2002} & \multirow[b]{2}{*}{2003} & \multirow[b]{2}{*}{2004} & \multicolumn{2}{|l|}{2005} & \multicolumn{2}{|l|}{2006} & \multicolumn{2}{|c|}{ Proj. } \\
\hline & & & & Report 06/123 & Est. & Report 06/123 & Proj. & 2007 & 2008 \\
\hline & \multicolumn{9}{|c|}{ (Percent change) } \\
\hline & \multicolumn{9}{|c|}{ I. Output, prices, and employment } \\
\hline Real GDP & -11.0 & 2.2 & 12.3 & 6.0 & 6.0 & 4.0 & 4.0 & 3.5 & 3.0 \\
\hline \multicolumn{10}{|l|}{ Contributions to real growth (percent) $1 /$} \\
\hline Consumption & -15.2 & -1.0 & 9.2 & 6.2 & 6.6 & 2.4 & 3.5 & 2.5 & 2.2 \\
\hline Investment & -5.0 & 2.7 & 2.9 & 1.9 & 1.1 & 5.7 & 4.9 & -0.9 & 0.4 \\
\hline Net exports & 9.1 & 0.9 & -1.2 & -2.2 & -1.7 & -4.0 & -4.3 & 1.9 & 0.4 \\
\hline GDP (Ur\$ billions) & 261 & 315 & 379 & 406 & 406 & 448 & 448 & 487 & 524 \\
\hline GDP (US\$ billions) & 12.1 & 11.2 & 13.3 & 16.5 & 16.5 & 18.3 & 18.3 & 19.5 & 20.6 \\
\hline Investment & 3.9 & 20.1 & 18.9 & 23.3 & 16.0 & 43.7 & 40.3 & -0.4 & 6.7 \\
\hline Consumption & -11.7 & 32.1 & 30.6 & 6.2 & 7.3 & 8.0 & 9.0 & 7.8 & 6.9 \\
\hline GDP deflator & 18.7 & 18.4 & 7.0 & 1.0 & 1.0 & 5.5 & 5.5 & 4.7 & 4.3 \\
\hline CPI inflation (average) & 14.0 & 19.4 & 9.2 & 4.9 & 4.7 & 6.2 & 6.0 & 4.9 & 4.4 \\
\hline CPI inflation (eop) & 25.9 & 10.2 & 7.6 & 5.9 & 4.9 & 5.5 & 5.5 & 4.7 & 4.4 \\
\hline Exchange rate change (Ur\$/US\$)(average) & 62.1 & 30.5 & 1.5 & $\ldots$ & -14.8 & $\ldots$ & $\ldots$ & $\ldots$ & $\ldots$ \\
\hline Exchange rate change (Ur\$/US\$) (eop) & 84.2 & 7.7 & -10.1 & ... & -8.5 & $\ldots$ & $\ldots$ & $\ldots$ & $\ldots$ \\
\hline Average public sector wage (end-of-period) & 0.5 & 7.9 & 9.7 & 10.2 & 10.2 & 10.5 & 10.5 & 9.6 & 8.6 \\
\hline \multirow[t]{2}{*}{ Unemployement (in percent) } & 17.0 & 16.9 & 13.1 & $\ldots$ & 12.1 & $\ldots$ & $\ldots$ & $\ldots$ & $\ldots$ \\
\hline & \multicolumn{9}{|c|}{ II. Monetary indicators } \\
\hline BCU monetary liabilities & -7.4 & 25.3 & 30.0 & 25.9 & 33.1 & 7.8 & -3.8 & 6.2 & 5.6 \\
\hline Base Money (average) & 17.7 & 19.3 & 7.7 & 22.1 & 28.5 & 11.1 & 4.6 & 8.6 & 7.5 \\
\hline Base Money (eop)2/ & 22.1 & 24.9 & 11.1 & 21.5 & 34.1 & 11.1 & 2.2 & 8.6 & 7.5 \\
\hline Currency issued & 5.8 & 22.4 & 15.7 & 21.5 & 22.0 & 11.1 & -0.2 & 8.6 & 7.5 \\
\hline M-1 & 1.7 & 34.6 & 13.4 & 22.0 & 29.4 & 11.1 & 7.0 & 8.6 & 7.5 \\
\hline M-2 & -5.1 & 17.4 & 12.8 & 16.7 & 22.3 & 11.1 & 6.9 & 8.6 & 7.5 \\
\hline M-3 & 15.8 & 21.7 & -2.0 & 6.0 & 0.1 & 9.0 & 1.7 & 6.1 & 6.3 \\
\hline \multirow[t]{3}{*}{ Credit to the private sector (constant exch. rate) $3 /$} & -17.6 & -23.9 & -11.2 & -4.0 & 2.7 & 9.9 & 9.9 & 10.8 & 6.9 \\
\hline & \multicolumn{9}{|c|}{ (Percent of GDP, unless otherwise indicated) } \\
\hline & \multicolumn{9}{|c|}{ III. Public sector operations } \\
\hline Revenue & 32.1 & 32.0 & 30.9 & 32.0 & 32.2 & 33.0 & 33.0 & 33.5 & 33.6 \\
\hline Non-interest expenditure (incl. discrepancy) & 32.1 & 29.3 & 27.2 & 28.4 & 28.4 & 29.3 & 29.3 & 29.5 & 29.6 \\
\hline Primary balance & 0.0 & 2.7 & 3.8 & 3.6 & 3.8 & 3.7 & 3.7 & 4.0 & 4.0 \\
\hline Interest & 4.7 & 6.0 & 6.0 & 4.8 & 4.6 & 4.8 & 4.8 & 4.6 & 4.5 \\
\hline Overall balance & -4.6 & -3.2 & -2.2 & -1.2 & -0.8 & -1.1 & -1.1 & -0.6 & -0.5 \\
\hline Public sector debt 4 / & 96 & 104 & 92 & 73 & 70 & 68 & 65 & 62 & 59 \\
\hline Public debt service (as a percent of GDP) & 13 & 14 & 18 & 15 & 16 & 16 & 17 & 13 & 11 \\
\hline
\end{tabular}


Table 1. Uruguay: Selected Economic and Social Indicators (concluded)

\begin{tabular}{|c|c|c|c|c|c|c|c|c|c|}
\hline & \multirow[b]{2}{*}{2002} & \multirow[b]{2}{*}{2003} & \multirow[b]{2}{*}{2004} & \multicolumn{2}{|l|}{2005} & \multicolumn{2}{|l|}{2006} & \multicolumn{2}{|c|}{ Proj. } \\
\hline & & & & Report $06 / 123$ & Est. & Report 06/123 & Proj. & 2007 & 2008 \\
\hline & \multicolumn{9}{|c|}{ IV. Savings and investment } \\
\hline Gross domestic investment & 11.5 & 12.6 & 13.3 & 15.7 & 14.8 & 20.6 & 18.9 & 17.4 & 17.2 \\
\hline Gross national savings & 14.7 & 12.1 & 12.6 & 13.3 & 12.7 & 14.7 & 13.3 & 13.7 & 14.2 \\
\hline \multirow[t]{2}{*}{ Foreign savings } & -3.2 & 0.5 & 0.7 & 2.4 & 2.0 & 5.8 & 5.6 & 3.6 & 3.1 \\
\hline & \multicolumn{9}{|c|}{ V. External indicators } \\
\hline Merchandise exports, fob (US\$ millions) & 1,922 & 2,281 & 3,021 & 3,490 & 3,484 & 3,769 & 3,760 & 4,089 & 4,443 \\
\hline Merchandise imports, fob (US\$ millions) & 1,874 & 2,098 & 2,990 & 3,840 & 3,797 & 4,854 & 4,767 & 4,747 & 5,015 \\
\hline Merchandise terms of trade (percentage change) & 4.3 & 2.9 & -3.1 & -9.3 & -9.9 & -3.8 & -3.0 & -1.5 & -1.2 \\
\hline Current account balance & 3.2 & -0.5 & -0.7 & -2.4 & -2.0 & -5.8 & -5.6 & -3.6 & -3.1 \\
\hline Of which: Excluding cellulose projects & 3.2 & -0.5 & -0.7 & -2.1 & -1.5 & -2.1 & -2.1 & -2.2 & -1.9 \\
\hline Foreign direct investment & 1.5 & 3.6 & 2.5 & 2.6 & 3.7 & 6.6 & 6.2 & 4.2 & 4.0 \\
\hline Overall balance of payments (US\$ millions) & $-2,328$ & 1,380 & 454 & 620 & 951 & 145 & 426 & 150 & 150 \\
\hline External debt 5/ & 87.5 & 98.2 & 87.4 & 71.0 & 68.4 & 62.9 & 61.2 & 57.3 & 53.7 \\
\hline Of which: External public debt & 68.9 & 85.3 & 76.9 & 63.1 & 61.1 & 55.9 & 54.7 & 51.3 & 47.9 \\
\hline External debt service (percent of exports of goods and services) & 55.0 & 52.3 & 40.9 & 50.6 & 51.0 & 53.7 & 60.3 & 45.4 & 37.6 \\
\hline Gross official reserves (US\$ millions) 6/ & 772 & 2,087 & 2,512 & 3,110 & 3,438 & 3,255 & 3,865 & 4,015 & 4,165 \\
\hline In months of imports of goods and services & 3.7 & 9.2 & 8.2 & 8.0 & 8.9 & 6.8 & 8.1 & 8.3 & 8.1 \\
\hline In percent of short-term debt & 25.1 & 86.8 & 158.0 & 135.8 & 156.7 & 125.2 & 137.5 & 195.3 & 231.2 \\
\hline In percent of short-term debt plus bank non-resident deposits & 14.3 & 45.1 & 61.6 & 64.6 & 76.6 & 62.4 & 74.2 & 88.2 & 94.7 \\
\hline In percent of short-term debt plus FX deposits & 7.0 & 20.0 & 27.7 & 29.7 & 33.1 & 28.8 & 34.4 & 37.2 & 38.5 \\
\hline REER (percentage depreciation -, e.o.p.) & -20.3 & -13.2 & 9.3 & $\ldots$ & 11.9 & $\ldots$ & $\ldots$ & $\ldots$ & $\ldots$ \\
\hline
\end{tabular}

Sources: Data provided by the Uruguayan authorities; and Fund staff estimates.

$1 / 2005$ and 2006 numbers are large driven by the large scale FDI project in the forestry sector.

2/ Program definition (end of period data).

3/ Part of the sharp drop in 2003 is due to the removal of the three liquidated banks from the database in May 2003.

4/ Covers debt of the NFPS and the central bank (excluding monetary policy instruments and free reserves).

5/ Excludes nonresident deposits.

6/ Includes reserve buildup through reserve requirements of resident financial institutions. 
Table 2. Uruguay: Performance under the 2005-06 Economic Program 1/

\begin{tabular}{|c|c|c|c|c|c|c|c|c|c|c|c|c|c|}
\hline & \multirow{2}{*}{$\frac{\text { Dec. } 31}{2004 \text { Stock }}$} & \multicolumn{4}{|c|}{ Jun. 30} & \multicolumn{3}{|c|}{ Sep. 30} & \multirow[b]{2}{*}{ Margin $(+)$} & \multicolumn{3}{|c|}{ Dec. 30} & \multirow[b]{2}{*}{ Margin (+) } \\
\hline & & $\mathrm{PC}$ & PC (adjusted) & Actual & Margin $(+)$ & $\mathrm{PC}$ & PC (adjusted) & Actual & & $\mathrm{PC}$ & PC (adjusted) & Prel. & \\
\hline \multicolumn{14}{|l|}{ A. Quantitative performance criteria } \\
\hline 1. Combined public sector primary balance (floor ) $2 /$ & $\ldots$ & 5,471 & 5,372 & 6,394 & 1,022 & 9,687 & 9,490 & 10,351 & 861 & 14,647 & 14,397 & 15,477 & 1,080 \\
\hline $\begin{array}{l}\text { 2. General government non-interest expenditure } \\
\text { (ceiling) } 2 /\end{array}$ & $\ldots$ & 23,561 & 23,539 & 22,080 & 1,459 & 34,643 & 34,665 & 32,996 & 1,670 & 46,561 & 46,683 & 45,137 & 1,546 \\
\hline 3. Net domestic assets of the BCU (ceiling) 2/ & 74,079 & 3,983 & $\begin{array}{l}3,983 \\
\text { (In mill }\end{array}$ & $\begin{array}{r}-302 \\
\text { ions of } U\end{array}$ & $\begin{array}{r}\mathbf{4 , 2 8 4} \\
\text { J.S. dollars) }\end{array}$ & 4,572 & 4,572 & $-16,424$ & 20,995 & $-3,910$ & $-8,416$ & $-22,449$ & 14,033 \\
\hline 4. Net international reserves of the BCU (floor) 2/ & $-2,218$ & -130 & -130 & 18 & 148 & -110 & -110 & 592 & 702 & 280 & 451 & 1,052 & 601 \\
\hline 5. Nonfinancial public sector gross debt (ceiling) $3 /$ & 12,189 & 12,510 & 12,495 & 12,229 & 266 & 12,575 & 12,566 & 12,496 & 70 & 12,550 & 12,745 & 12,650 & 95 \\
\hline $\begin{array}{l}\text { 6. Accumulation of external payment arrears of } \\
\text { the public sector (on a continuous basis) } 2 /\end{array}$ & 0 & 0 & 0 & 0 & $\mathbf{0}$ & 0 & 0 & 0 & $\mathbf{0}$ & 0 & 0 & 0 & 0 \\
\hline \multicolumn{14}{|c|}{ (In millions of Uruguayan pesos) } \\
\hline 8. Monetary base (ceiling) $2 /$ & 15,648 & 557 & 557 & 1,070 & -513 & 1,673 & 1,673 & 498 & 1,175 & 3,468 & 3,468 & 4,460 & -992 \\
\hline 9. Floating debt of the central government (ceiling) & 3,081 & 3,081 & 3,081 & 2,567 & 514 & 3,081 & 3,081 & 2,298 & 783 & 3,081 & 3,081 & 2,482 & 599 \\
\hline
\end{tabular}


Table 2. Uruguay: Performance under the 2005-06 Economic Program (concluded) 1/

\begin{tabular}{|c|c|c|c|}
\hline Area & Structural Conditionality & Date & Status \\
\hline $\begin{array}{l}\text { A. Prior action } \\
\text { Tax reform }\end{array}$ & Submit to congress a comprehensive tax reform as described in paragraph 7 . & & \\
\hline \multicolumn{4}{|c|}{ B. Structural performance criteria } \\
\hline Fiscal & $\begin{array}{l}\text { Submit to congress a five-year spending plan, complemented with revenue projections and deficit targets consistent with the program's fiscal } \\
\text { targets. }\end{array}$ & August 31,2005 & Observed. \\
\hline Fiscal & $\begin{array}{l}\text { Have in place a five-year spending plan, complemented with revenue projections and deficit targets consistent with the program's fiscal } \\
\text { targets. }\end{array}$ & February 28, 2006 & Observed. \\
\hline Tax reform & Submit to congress a comprehensive tax reform as described in paragraph 7 . & February 28, 2006 & $\begin{array}{r}\text { Not observed; reset } \\
\text { as PA. }\end{array}$ \\
\hline Tax reform & Begin to implement the comprehensive tax reform. & June 30,2006 & \\
\hline Central Bank & $\begin{array}{l}\text { Submit simultaneously three laws to congress to: (i) give appropriate autonomy to the central bank (as described in paragraph } 12 \text { of the } \\
\text { MEFP); (ii) strengthen the regulation of the financial system (as described in paragraph } 13 \text { of the MEFP); and (iii) provide a suitable bank } \\
\text { resolution framework (as described in paragraph } 14 \text { of the MEFP). }\end{array}$ & December 31, 2005 & Not observed. 4 \\
\hline BROU \& BHU & Government to ensure timely service of BHU note and BROU fiduciary notes to BROU in accordance with the current payment schedules. & Continuous & Observed. \\
\hline BHU & Adopt action plan to address the financial situation of BHU consistent with minimizing systemic risks and contingent fiscal costs. & December 31, 2005 & Observed. \\
\hline Pensions & Begin to implement reform of the pension fund for the police. & May 31, 2006 & \\
\hline Pensions & Submit to Congress reform of the pension fund for the military and bank employees as described in paragraph 12 of the SMEFP. & November 30, 2006 & \\
\hline Pensions & Begin to implement the reform of the pension fund for the military and bank employees as described in paragraph 12 of the SMEFP. & May 31,2007 & \\
\hline \multicolumn{4}{|c|}{ C. Structural benchmarks } \\
\hline Fiscal & Establish quarterly revenue collection targets (floors) at the social security bank (BPS). & June 30,2005 & Observed. \\
\hline Debt management & Create a debt management unit at the Ministry of Finance. & December 31, 2005 & Observed. \\
\hline Tax administration & $\begin{array}{l}\text { Sign a memorandum of understanding between the Ministry of Finance and the DGI agreeing on quantitative targets and indicators for } 2006 \\
\text { on tax collections, audit coverage, tax services, and information systems. }\end{array}$ & December 31, 2005 & Observed. \\
\hline Tax administration & Formulate a plan to strengthen the auditing and enforced collection functions of the BPS. & June 30, 2006 & \\
\hline Tax administration & $\begin{array}{l}\text { Finalize the design of a comprehensive reform plan for the customs agency (including establishing collection targets consistent with the } \\
\text { program). }\end{array}$ & August 31,2006 & \\
\hline Debt management & Create a debt management unit at the Ministry of Finance. & December 31, 2005 & \\
\hline Budget & $\begin{array}{l}\text { Prepare recommendations, with a timetable, to improve legislation, coverage, classification, formulation, controls, and transparency of the } \\
\text { budget process. }\end{array}$ & August 31, 2006 & \\
\hline Growth & Publish agenda of growth-enhancing reforms (including timetable for implementation) prepared by the business environment commission. & March 31, 2006 & \\
\hline Growth & Submit to Congress bankruptcy law (to include Chapter-11 type corporate restructuring). & June 30, 2006 & \\
\hline Financial sector & Adopt a detailed schedule for the implementation of the BHU action plan. & February 28, 2006 & Observed. \\
\hline Financial sector & Sell shares of NBC in amounts that yield managerial control to the private sector. & June 30, 2006 & \\
\hline Central Bank & Adopt plan to strengthen the central bank finances (outright capitalization or interest payment on government paper). & September 30, 2006 & \\
\hline
\end{tabular}

CInternational Monetary Fund. Not for Redistribution 
Table 3. Uruguay: Balance of Payments (In millions of US\$, unless otherwise stated)

\begin{tabular}{|c|c|c|c|c|c|c|c|c|c|}
\hline & \multirow[b]{2}{*}{2002} & \multirow[b]{2}{*}{2003} & \multirow[b]{2}{*}{2004} & \multicolumn{2}{|l|}{2005} & \multicolumn{2}{|l|}{2006} & \multicolumn{2}{|c|}{ Proj. } \\
\hline & & & & Report 06/123 & Est. & Report $06 / 123$ & Proj. & 2007 & 2008 \\
\hline & \multicolumn{9}{|c|}{ 1. Balance of Payments } \\
\hline Current account & 382 & -56 & -98 & -399 & -342 & $-1,100$ & $-1,063$ & -729 & -652 \\
\hline Trade balance & 48 & 183 & 31 & -351 & -313 & $-1,084$ & $-1,007$ & -658 & -572 \\
\hline Exports, f.o.b. & 1,922 & 2,281 & 3,021 & 3,490 & 3,484 & 3,769 & 3,760 & 4,089 & 4,443 \\
\hline Imports, f.o.b. & 1,874 & 2,098 & 2,990 & 3,840 & 3,797 & 4,854 & 4,767 & 4,747 & 5,015 \\
\hline Services & 153 & 167 & 304 & 300 & 357 & 381 & 385 & 365 & 353 \\
\hline Exports, f.o.b. & 771 & 803 & 987 & 1,148 & 1,220 & 1,291 & 1,316 & 1,391 & 1,483 \\
\hline Imports, f.o.b. & 618 & 636 & 683 & 849 & 863 & 910 & 931 & 1,026 & 1,131 \\
\hline Income (net) & 109 & -489 & -522 & -437 & -484 & -489 & -543 & -543 & -546 \\
\hline Transfers (net) & 72 & 83 & 89 & 89 & 97 & 93 & 102 & 107 & 114 \\
\hline Financial and capital account & $-2,234$ & 1,039 & 163 & 650 & 869 & 1,296 & 1,489 & 879 & 802 \\
\hline Foreign direct investment & 180 & 401 & 337 & 429 & 618 & 1,250 & 1,174 & 840 & 838 \\
\hline Portfolio investment & 415 & -541 & -416 & 575 & 768 & 39 & 558 & 70 & 246 \\
\hline Government securities & 171 & -5 & 266 & 547 & 560 & 181 & 687 & 108 & 275 \\
\hline Issues & 710 & 613 & 532 & 992 & 1,008 & 597 & 1,101 & 226 & 373 \\
\hline Amortization 1/ & 539 & 618 & 266 & 445 & 447 & 416 & 415 & 118 & 98 \\
\hline Banks & 244 & -537 & -682 & 28 & 207 & -142 & -129 & -37 & -28 \\
\hline Other capital flows (net) & $-2,828$ & 1,179 & 242 & -354 & -517 & 6 & -242 & -32 & -283 \\
\hline Loans & 1,290 & 375 & -92 & -285 & -241 & -161 & -618 & -160 & -400 \\
\hline Of which: WB, IDB, commercial (net) & 633 & 237 & -36 & 6 & 17 & -73 & -433 & 229 & -11 \\
\hline Disbursements & 792 & 472 & 217 & 370 & 386 & 432 & 307 & 567 & 379 \\
\hline Amortization & 159 & 235 & 253 & 364 & 370 & 505 & 741 & 339 & 390 \\
\hline Of which: IMF (net) & 883 & 671 & 152 & -177 & -176 & -88 & -135 & -389 & -389 \\
\hline Disbursements & 1,989 & 840 & 552 & 303 & 304 & 544 & 536 & 387 & 88 \\
\hline Amortization & 1,105 & 169 & 400 & 480 & 480 & 632 & 670 & 775 & 477 \\
\hline Deposits, net & $-1,693$ & 267 & 180 & -239 & -428 & 0 & 0 & 0 & 0 \\
\hline Other flows, net & $-2,426$ & 537 & 154 & 170 & 152 & 167 & 376 & 128 & 118 \\
\hline Errors and omissions & -476 & 397 & 388 & 419 & 424 & 0 & 0 & 0 & 0 \\
\hline Overall balance & $-2,328$ & 1,380 & 454 & 620 & 951 & 145 & 426 & 150 & 150 \\
\hline \multirow[t]{2}{*}{ Reserve assets (- increase) } & 2,328 & $-1,380$ & -454 & -620 & -951 & -145 & -426 & -150 & -150 \\
\hline & \multicolumn{9}{|c|}{ 2. Reserve Adequacy and External Indicators } \\
\hline Gross official reserves (stock) & 772 & 2,087 & 2,512 & 3,110 & $3, \mathbf{4 3 8}$ & 3,255 & 3,865 & 4,015 & 4,165 \\
\hline In months of imports of goods and services & 3.7 & 9.2 & 8.2 & 8.0 & 8.9 & 6.8 & 8.1 & 8.3 & 8.1 \\
\hline In percent of short-term debt excluding nonresidential deposits & 25.1 & 86.8 & 158.0 & 135.8 & 156.7 & 125.2 & 137.5 & 195.3 & 231.2 \\
\hline In percent of short-term debt including nonresidential deposits & 14.3 & 45.1 & 61.6 & 64.6 & 76.6 & 62.4 & 74.2 & 88.2 & 94.7 \\
\hline \multirow[t]{2}{*}{ Net international reserves (stock) 2/ } & $-1,088$ & -763 & $-2,218$ & $-1,303$ & $-1,166$ & -992 & -843 & -299 & 197 \\
\hline & \multicolumn{9}{|c|}{ (As percent of GDP) } \\
\hline Exports & 15.9 & 20.3 & 22.8 & 20.9 & 20.8 & 19.9 & 19.9 & 20.4 & 20.9 \\
\hline Imports & 15.5 & 18.7 & 22.5 & 23.0 & 22.7 & 25.7 & 25.2 & 23.6 & 23.6 \\
\hline Current account & 3.2 & -0.5 & -0.7 & -2.4 & -2.0 & -5.8 & -5.6 & -3.6 & -3.1 \\
\hline Underlying current account 3 / & 3.2 & -0.5 & -0.7 & -2.1 & -1.5 & -2.1 & -2.1 & -2.2 & -1.9 \\
\hline Financial and capital account & -18.5 & 9.3 & 1.2 & 3.9 & 5.2 & 6.9 & 7.9 & 4.4 & 3.8 \\
\hline Of which: foreign direct investment (net) & 1.5 & 3.6 & 2.5 & 2.6 & 3.7 & 6.6 & 6.2 & 4.2 & 4.0 \\
\hline Of which: government securities (net) & 1.4 & 0.0 & 2.0 & 3.3 & 3.3 & 1.0 & 3.6 & 0.5 & 1.3 \\
\hline Of which: IMF (net) & 7.3 & 6.0 & 1.1 & -1.1 & -1.1 & -0.5 & -0.7 & -1.9 & -1.8 \\
\hline Overall balance & -19.3 & 12.3 & 3.4 & 3.7 & 5.7 & 0.8 & 2.3 & 0.7 & 0.7 \\
\hline Changes in GIR & 19.3 & -12.3 & -3.4 & -3.7 & -5.7 & -0.8 & -2.3 & -0.7 & -0.7 \\
\hline External debt & 87.5 & 98.2 & 87.4 & 71.0 & 68.4 & 62.9 & 61.2 & 57.3 & 53.7 \\
\hline Short-term debt (residual maturity) & 25.5 & 21.4 & 12.0 & 13.7 & 13.1 & 13.7 & 14.9 & 10.2 & 8.5 \\
\hline External public debt & 68.9 & 85.3 & 76.9 & 63.1 & 61.1 & 55.9 & 54.7 & 51.3 & 47.9 \\
\hline External debt + NR deposits & 106.8 & 118.1 & 106.1 & 86.1 & 82.2 & 76.8 & 73.8 & 69.8 & 65.9 \\
\hline & & & (As pe & ercent of annual & xports of & goods and servi & & & \\
\hline Total external debt & 392.8 & 357.1 & 289.3 & 256.1 & 243.3 & 235.1 & 227.8 & 210.1 & 192.2 \\
\hline Total external debt (including nonresidential deposits) & 479.2 & 429.3 & 351.3 & 310.4 & 292.1 & 286.9 & 275.0 & 255.7 & 236.0 \\
\hline Debt service & 55.0 & 52.3 & 40.9 & 50.6 & 51.0 & 53.7 & 60.3 & 45.4 & 37.6 \\
\hline Of which: interest payments & 24.5 & 19.2 & 18.0 & 17.6 & 18.2 & 18.8 & 20.5 & 19.4 & 18.0 \\
\hline & & & & (Annual & ercent ch & langes) & & & \\
\hline Exports & -10.2 & 18.7 & 32.4 & 15.5 & 15.3 & 8.0 & 7.9 & 8.7 & 8.7 \\
\hline Imports & -35.7 & 12.0 & 42.5 & 28.4 & 27.0 & 26.4 & 25.5 & -0.4 & 5.6 \\
\hline Export prices in US\$ (year-on-year percent change) 3/ & -7.7 & 7.4 & 6.5 & 4.5 & 4.8 & 0.5 & 1.1 & 0.1 & -0.8 \\
\hline Import prices in US\$ (year-on-year percent change) 3/ & -10.8 & 5.1 & 9.2 & 11.6 & 12.4 & 2.5 & 2.4 & 0.6 & -0.3 \\
\hline Terms of trade 3 / & 3.5 & 2.2 & -2.5 & -6.3 & -6.7 & -1.9 & -1.3 & -0.5 & -0.5 \\
\hline Export volume 3/ & -2.1 & 9.4 & 23.6 & 12.3 & 12.2 & 9.5 & 8.6 & 7.0 & 7.2 \\
\hline Import volume 3/ & -26.9 & 6.2 & 29.0 & 11.9 & 8.5 & 6.6 & 8.2 & 8.3 & 8.1 \\
\hline
\end{tabular}

Sources: Central Bank of Uruguay; and Fund staff estimates and projections.

1/ Includes secondary market transactions between residents and non-residents.

2/ Includes all liabilities to the Fund and liabilities to residents; follows respective TMU definitions.

3/ Excluding imports related to the construction of planned pulp mill projects (Botnia and ENCE). 
Table 4. Uruguay: Public Sector Operations

(In millions of pesos)

\begin{tabular}{|c|c|c|c|c|c|c|c|c|c|c|}
\hline & \multirow[b]{2}{*}{2002} & \multirow[b]{2}{*}{2003} & \multirow[b]{2}{*}{2004} & \multicolumn{2}{|l|}{2005} & \multicolumn{2}{|l|}{2006} & \multicolumn{3}{|c|}{ Proj. } \\
\hline & & & & Report 06/123 & Prel. & Report 06/123 & Proj. & 2007 & 2008 & 2009 \\
\hline Revenue & 83,891 & 101,081 & 117,367 & 129,840 & 130,665 & 146,832 & 147,041 & 161,857 & 174,186 & 187,414 \\
\hline Taxes & 57,832 & 70,096 & 83,002 & 91,787 & 92,118 & 101,770 & 101,843 & 113,093 & 121,263 & 129,714 \\
\hline VAT and excise taxes & 29,708 & 37,667 & 47,200 & 53,015 & 53,124 & 60,444 & 60,468 & 65,541 & 70,316 & 74,976 \\
\hline On income and profits & 14,302 & 15,621 & 17,683 & 19,873 & 20,124 & 21,280 & 21,336 & 26,707 & 29,831 & 32,321 \\
\hline On foreign trade & 2,730 & 3,780 & 4,958 & 5,099 & 5,181 & 5,090 & 5,108 & 4,195 & 3,829 & 4,103 \\
\hline On property and other & 11,091 & 13,028 & 13,161 & 13,800 & 13,689 & 14,955 & 14,931 & 16,650 & 17,287 & 18,314 \\
\hline Social security contributions $1 /$ & 15,589 & 14,696 & 16,657 & 20,941 & 21,532 & 23,192 & 23,289 & 25,067 & 27,027 & 28,918 \\
\hline Nontax revenue & 5,274 & 6,200 & 7,831 & 8,259 & 8,402 & 9,818 & 9,857 & 10,339 & 11,050 & 11,742 \\
\hline Current surplus of public enterprises & 5,196 & 10,090 & 9,876 & 8,854 & 8,614 & 12,052 & 12,052 & 13,359 & 14,846 & 17,041 \\
\hline Noninterest expenditure & 83,819 & 92,431 & 103,082 & 115,179 & 115,187 & 130,360 & 130,401 & 142,547 & 153,437 & 165,262 \\
\hline Current 2/ & 77,405 & 84,623 & 93,780 & 105,757 & 105,766 & 117,243 & 117,284 & 126,156 & 135,935 & 146,250 \\
\hline Wages & 18,158 & 19,701 & 23,409 & 25,768 & 25,454 & 28,722 & 28,722 & 31,869 & 34,941 & 38,141 \\
\hline Goods and services & 12,057 & 15,031 & 17,015 & 19,319 & 19,464 & 22,642 & 22,642 & 23,359 & 24,703 & 26,645 \\
\hline Of Which: SEP & 0 & 0 & 0 & 879 & 780 & 2,553 & 2,553 & 1,548 & 0 & 0 \\
\hline Social security benefits 1 / & 42,818 & 44,218 & 47,702 & 53,019 & 53,057 & 57,626 & 57,667 & 62,558 & 67,236 & 71,781 \\
\hline Other 1/ & 4,371 & 5,673 & 5,654 & 7,651 & 7,640 & 8,254 & 8,254 & 8,369 & 9,055 & 9,684 \\
\hline Capital (Government and enterprises) & 6,413 & 7,808 & 9,303 & 9,422 & 9,572 & 13,117 & 13,117 & 16,391 & 17,502 & 19,011 \\
\hline Primary balance & 72 & 8,651 & 14,284 & 14,662 & 15,477 & 16,473 & 16,640 & 19,311 & 20,748 & 22,152 \\
\hline Interest & 12,163 & 18,881 & 22,666 & 19,402 & 18,747 & 21,455 & 21,455 & 22,276 & 23,285 & 24,293 \\
\hline Overall balance & $-12,091$ & $-10,231$ & $-8,382$ & $-4,741$ & $-3,269$ & $-4,983$ & $-4,815$ & $-2,966$ & $-2,537$ & $-2,141$ \\
\hline
\end{tabular}


Table 4. Uruguay: Public Sector Operations (continued)

(In percent of GDP)

\begin{tabular}{|c|c|c|c|c|c|c|c|c|c|c|}
\hline & \multirow[b]{2}{*}{2002} & \multirow[b]{2}{*}{2003} & \multirow[b]{2}{*}{2004} & \multicolumn{2}{|l|}{2005} & \multicolumn{2}{|l|}{2006} & \multicolumn{3}{|c|}{ Proj. } \\
\hline & & & & Report $06 / 123$ & Prel. & Report $06 / 123$ & Proj. & 2007 & 2008 & 2009 \\
\hline Revenue & 32.1 & 32.0 & 30.9 & 32.0 & 32.2 & 33.0 & 33.0 & 33.5 & 33.6 & 33.8 \\
\hline Taxes & 22.2 & 22.2 & 21.9 & 22.6 & 22.7 & 22.8 & 22.9 & 23.4 & 23.4 & 23.4 \\
\hline VAT and excise taxes & 11.4 & 11.9 & 12.4 & 13.1 & 13.1 & 13.6 & 13.6 & 13.6 & 13.6 & 13.5 \\
\hline On income and profits & 5.5 & 5.0 & 4.7 & 4.9 & 5.0 & 4.8 & 4.8 & 5.5 & 5.8 & 5.8 \\
\hline On foreign trade & 1.0 & 1.2 & 1.3 & 1.3 & 1.3 & 1.1 & 1.1 & 0.9 & 0.7 & 0.7 \\
\hline On property and other & 4.3 & 4.1 & 3.5 & 3.4 & 3.4 & 3.4 & 3.4 & 3.45 & 3.33 & 3.31 \\
\hline Social security contributions 1 / & 6.0 & 4.7 & 4.4 & 5.2 & 5.3 & 5.2 & 5.2 & 5.2 & 5.2 & 5.2 \\
\hline Nontax revenue & 2.0 & 2.0 & 2.1 & 2.0 & 2.1 & 2.2 & 2.2 & 2.1 & 2.1 & 2.1 \\
\hline Current surplus of public enterprises & 2.0 & 3.2 & 2.6 & 2.2 & 2.1 & 2.7 & 2.7 & 2.8 & 2.9 & 3.1 \\
\hline Non-interest expenditure & 32.1 & 29.3 & 27.2 & 28.4 & 28.4 & 29.3 & 29.3 & 29.5 & 29.6 & 29.8 \\
\hline Current & 29.7 & 26.8 & 24.7 & 26.0 & 26.0 & 26.3 & 26.3 & 26.1 & 26.2 & 26.4 \\
\hline Wages & 7.0 & 6.2 & 6.2 & 6.3 & 6.3 & 6.4 & 6.4 & 6.6 & 6.7 & 6.9 \\
\hline Goods and services & 4.6 & 4.8 & 4.5 & 4.8 & 4.8 & 5.1 & 5.1 & 4.8 & 4.8 & 4.8 \\
\hline Of which: SEP & 0.0 & 0.0 & 0.0 & 0.2 & 0.2 & 0.6 & 0.6 & 0.3 & 0.0 & 0.0 \\
\hline Social security benefits 1 / & 16.4 & 14.0 & 12.6 & 13.1 & 13.1 & 12.9 & 12.9 & 13.0 & 13.0 & 2113. \\
\hline Other 1/ & 1.7 & 1.8 & 1.5 & 1.9 & 1.9 & 1.9 & 1.9 & 1.7 & 1.7 & 1.7 \\
\hline Capital (government and enterprises) & 2.5 & 2.5 & 2.5 & 2.3 & 2.4 & 2.9 & 2.9 & 3.4 & 3.4 & 3.4 \\
\hline Primary balance & 0.0 & 2.7 & 3.8 & 3.6 & 3.8 & 3.7 & 3.7 & 4.0 & 4.0 & 4.0 \\
\hline Interest & 4.7 & 6.0 & 6.0 & 4.8 & 4.6 & 4.8 & 4.8 & 4.6 & 4.5 & 4.4 \\
\hline Overall balance & -4.6 & -3.2 & -2.2 & -1.2 & -0.8 & -1.1 & -1.1 & -0.6 & -0.5 & -0.4 \\
\hline \multicolumn{11}{|l|}{ Memorandum items: } \\
\hline Augmented balance $\mathbb{2}$ & $-56,106$ & $-10,231$ & $-1,229$ & $-7,072$ & $-3,269$ & $-6,399$ & $-5,759$ & $-2,966$ & $-2,537$ & $-2,141$ \\
\hline In percent of GDP & -21.5 & -3.2 & -0.3 & -1.7 & -0.8 & -1.4 & -1.3 & -0.6 & -0.5 & -0.4 \\
\hline GDP (billions of pesos) & 261 & 315 & 379 & 406 & 406 & 445 & 445 & 483 & 519 & 554 \\
\hline
\end{tabular}

Sources: Ministry of Finance; and Fund staff estimates.

1/ This line was amended at the time of the second review to incorporate contributions collected by the BPS transferred to the AFAPs previously excluded.

2/ In 2002 this includes the following bank-restructuring costs: US $\$ 33$ million of capital transfers for bank recapitalization, US\$564 million of liquidity supplied by BCU,

US\$444 million for the Fondo de Fortalecimiento del Sistema Bancario (FFSB), and US\$993 million for the FSBS. Asset recoveries are credited in 2004. In 2005 and 2006

this includes US\$60 million for the financing of the deposit insurance scheme. Contingent liabilities of BHU will be included once the estimates are finalized. 
Table 5. Uruguay: Summary Accounts of the Banking System 1/ (In millions of US dollars)

\begin{tabular}{|c|c|c|c|c|c|c|c|}
\hline & \multirow[b]{2}{*}{2002} & \multirow[b]{2}{*}{2003} & \multirow[b]{2}{*}{2004} & \multicolumn{2}{|c|}{2005} & \multicolumn{2}{|l|}{2006} \\
\hline & & & & Report $06 / 123$ & Est. & Report $06 / 123$ & Proj \\
\hline & \multicolumn{7}{|c|}{ 1. Banco Central del Uruguay } \\
\hline Net foreign assets & -689 & -52 & -51 & 1,005 & 1,307 & 1,244 & 1,382 \\
\hline Net international reserves $2 /$ & $-1,014$ & -321 & -164 & 766 & 1,135 & 995 & 1,176 \\
\hline Gross international reserves & 772 & 2,087 & 2,512 & 3,110 & 3,439 & 3,255 & 3,491 \\
\hline Reserve liabilities $2 /$ & $-1,786$ & $-2,407$ & $-2,675$ & $-2,344$ & $-2,304$ & $-2,260$ & $-2,315$ \\
\hline Other net foreign assets & 325 & 269 & 113 & 239 & 172 & 249 & 206 \\
\hline Net domestic assets & 1,123 & 532 & 652 & -295 & -287 & -455 & -505 \\
\hline Net credit to the public sector & 1,541 & 2,464 & 2,626 & 2,023 & 1,969 & 1,777 & 1,755 \\
\hline Net credit to the financial system & -340 & $-2,112$ & $-1,905$ & $-1,879$ & $-1,975$ & $-1,865$ & $-2,054$ \\
\hline Credit to the private sector & 80 & 71 & 62 & 56 & 65 & 46 & 66 \\
\hline Securities issued by the BCU & -34 & -243 & -563 & -667 & -904 & -663 & $-1,079$ \\
\hline Other & -124 & 352 & 431 & 172 & 558 & 250 & 808 \\
\hline Peso monetary liabilities & 434 & 480 & 600 & 710 & 1,020 & 789 & 877 \\
\hline \multicolumn{8}{|l|}{ Memorandum items: 3 / } \\
\hline Monetary base (average) 4/ & $\ldots$ & $\ldots$ & 594 & 725 & 763 & 880 & 880 \\
\hline Monetary base (end of period) 4/ & & & 594 & 714 & 784 & 866 & 866 \\
\hline NIR program definition & $\ldots$ & $\ldots$ & $-2,218$ & $-1,316$ & $-1,166$ & -992 & -843 \\
\hline NDA program definition & $\ldots$ & $\ldots$ & 2,811 & 2,030 & 1,950 & 1,858 & 1,709 \\
\hline NIR flows & $\ldots$ & $\ldots$ & $\ldots$ & $\ldots$ & 1,052 & $\ldots$ & 243 \\
\hline NDA flows & $\ldots$ & $\ldots$ & $\ldots$ & $\ldots$ & -852 & $\ldots$ & -241 \\
\hline \multicolumn{8}{|c|}{ 2. Public and Private Banks 5/ } \\
\hline Net foreign assets & 551 & 1,213 & 1,887 & 2,271 & 2,030 & 2,413 & 2,090 \\
\hline Net domestic assets & 5,254 & 5,331 & 5,090 & 5,272 & 5,397 & 5,699 & 5,839 \\
\hline Net credit to the public sector 1 / & 399 & 219 & 437 & 345 & 0 & 222 & -124 \\
\hline Net credit to the financial system & 947 & 2,612 & 2,256 & 2,317 & 2,602 & 2,553 & 2,756 \\
\hline Credit to the private sector & 6,319 & 4,726 & 4,307 & 4,527 & 4,533 & 4,892 & 4,996 \\
\hline Other & $-2,411$ & $-2,226$ & $-1,909$ & $-1,918$ & $-1,738$ & $-1,968$ & $-1,788$ \\
\hline Liabilities to the private sector (residents) & 5,805 & 6,544 & 6,977 & 7,543 & 7,426 & 8,112 & 7,930 \\
\hline Public banks & 3,140 & 3,409 & 3,670 & 3,896 & 3,842 & 4,156 & 4,070 \\
\hline Local currency & 339 & 394 & 520 & 598 & 682 & 663 & 727 \\
\hline Foreign currency & 2,801 & 3,016 & 3,149 & 3,299 & 3,160 & 3,494 & 3,343 \\
\hline Private banks & 2,666 & 3,135 & 3,307 & 3,647 & 3,584 & 3,956 & 3,860 \\
\hline Local currency & 357 & 349 & 405 & 477 & 574 & 531 & 583 \\
\hline Foreign currency & 2,309 & 2,785 & 2,902 & 3,169 & 3,010 & 3,424 & 3,276 \\
\hline \multicolumn{8}{|c|}{ 3. Banking System } \\
\hline Net foreign assets & -138 & 1,161 & 1,835 & 3,276 & 3,337 & 3,657 & 3,524 \\
\hline Net domestic assets & 6,288 & 5,788 & 5,738 & 4,755 & 4,952 & 5,093 & 4,999 \\
\hline Credit to the public sector & 1,940 & 2,684 & 3,063 & 2,369 & 1,970 & 1,999 & 1,631 \\
\hline Credit to the rest of financial system & -218 & -173 & -165 & 216 & 470 & 538 & 419 \\
\hline Credit to the private sector & 6,398 & 4,797 & 4,369 & 4,583 & 4,598 & 4,938 & 5,061 \\
\hline Other & $-1,831$ & $-1,519$ & $-1,529$ & $-2,413$ & $-2,086$ & $-2,382$ & $-2,112$ \\
\hline Broad money (M3) & 6,150 & 6,949 & 7,573 & 8,030 & 8,288 & 8,750 & 8,524 \\
\hline Memorandum items: & \multicolumn{7}{|c|}{ (Percentage chage) } \\
\hline Base money (average) & 18 & 19 & 8 & 22 & 28 & 11 & 5 \\
\hline Base money (eop) & 22 & 25 & 11 & 22 & 34 & 11 & 2 \\
\hline Currency issued & 6 & 22 & 16 & 22 & 22 & 11 & 0 \\
\hline M-1 & 2 & 35 & 13 & 22 & 29 & 11 & 7 \\
\hline M-2 & -5 & 17 & 13 & 17 & 22 & 11 & 7 \\
\hline M-3 & 16 & 22 & -2 & 6 & 0 & 9 & 2 \\
\hline
\end{tabular}

Sources: Central Bank of Uruguay; and Fund staff estimates and projections.

1/ Presentation used for program monitoring. May differ from presentation and definitions used in IFS.

2/ Includes all outstanding liabilities to the IMF, but excludes liabilities to resident financial institutions.

3/ Program figures for 2005 are estimated at December 2004 exchange rates, while those for 2006 are estimated at September 2005 exchange rates.

4/ Monetary base excludes from peso monetary liabilities the net government and BPS deposits with BROU, which are subject to 100 percent requirements.

5/ The Banco de la Republica Oriental de Uruguay (BROU), Banco Hipotecario de Uruguay

(BHU; mortgage institution), private banks, and cooperatives. 
Table 6. Uruguay: Vulnerability Indicators

\begin{tabular}{|c|c|c|c|c|c|}
\hline & 2002 & 2003 & 2004 & $\begin{array}{r}\text { Est. } \\
2005\end{array}$ & $\begin{array}{l}\text { Proj. } \\
2006\end{array}$ \\
\hline \multicolumn{6}{|c|}{ (Percent change, unless otherwise indicated) } \\
\hline \multicolumn{6}{|l|}{ Financial sector indicators } \\
\hline Broad money & 21.3 & 24.2 & -9.5 & 0.1 & 0.8 \\
\hline Credit to the private sector (const. exch. rate) & -17.6 & -23.9 & -11.2 & 2.7 & 10.0 \\
\hline Share of nonperforming loans in total loans (in percent) & 31.5 & 8.6 & 3.8 & 2.5 & $\ldots$ \\
\hline Provisions to nonperforming loans & $\ldots$ & $\ldots$ & 56.2 & 50.8 & ... \\
\hline Capital adequacy ratio (in percent) & -9.6 & 9.2 & 10.9 & 11.2 & $\ldots$ \\
\hline Return on assets & -25.6 & -1.1 & -0.2 & 0.7 & $\ldots$ \\
\hline Interbank interest rates (percent, end-of-period) & 45.0 & 2.5 & 1.3 & 1.0 & $\cdots$ \\
\hline FX deposits held by residents (in percent of total deposits) & 88.3 & 88.8 & 87.6 & 83.7 & 85.0 \\
\hline FX loans to residents (in percent of total loans) & 80.4 & 77.6 & 73.3 & 70.0 & 70.0 \\
\hline \multicolumn{6}{|l|}{ Public debt indicators } \\
\hline Public sector gross debt (PSGD, in percent of GDP) & 95.5 & 104.5 & 92.5 & 69.9 & 65.3 \\
\hline Of which: Exposed to rollover risk (in percent of total PSGD) & 17.2 & 12.1 & 13.5 & 16.0 & $\ldots$ \\
\hline Exposed to exchange rate risk (in percent of total PSGD) & 96.0 & 94.3 & 90.2 & 87.0 & $\ldots$ \\
\hline Exposed to interest rate risk (in percent of total PSGD) & 57.0 & 45.5 & 44.6 & 40.0 & ... \\
\hline \multicolumn{6}{|l|}{ External indicators } \\
\hline Merchandise exports & -10.2 & 18.7 & 32.4 & 15.3 & 7.9 \\
\hline Merchandise imports & -35.7 & 12.0 & 42.5 & 27.0 & 25.5 \\
\hline Merchandise terms of trade & 4.3 & 2.9 & -3.1 & -9.9 & -3.0 \\
\hline REER appreciation $(+)$ & -20.3 & -13.2 & 9.3 & 11.9 & ... \\
\hline \multicolumn{6}{|c|}{ (Percent of GDP, unless otherwise indicated) } \\
\hline Current account balance & 3.2 & -0.5 & -0.7 & -2.0 & -5.6 \\
\hline Capital and financial account balance & -18.5 & 9.3 & 1.2 & 5.2 & 7.9 \\
\hline Of which: Net foreign direct investment & 1.5 & 3.6 & 2.5 & 3.7 & 6.2 \\
\hline Government securities & 1.4 & 0.0 & 2.0 & 3.3 & 3.6 \\
\hline Other net inflows & -21.4 & 5.7 & -3.3 & -1.9 & -2.0 \\
\hline Total gross external debt (ED) in percent of GDP & 87.5 & 98.2 & 87.4 & 68.4 & 61.2 \\
\hline Of which: Short-term ED (original maturity, in percent of total ED) & 12.5 & 6.7 & 7.6 & 4.6 & 5.2 \\
\hline ED to foreign official sector (in percent of total ED) & 44.9 & 51.1 & 50.7 & 49.4 & 44.0 \\
\hline External interest payments (in percent of exports of GNFS) & 24.5 & 19.2 & 18.0 & 18.2 & 20.5 \\
\hline External amortization payments (in percent of exports of GNFS) & 30.5 & 33.1 & 22.8 & 32.8 & 39.8 \\
\hline \multicolumn{6}{|c|}{ (U.S. million, unless otherwise indicated) } \\
\hline Central Bank reserve liabilities 1/ & 1,786 & 2,407 & 2,675 & 2,334 & 2,260 \\
\hline Short-term foreign assets of the banking system & 3,140 & 4,989 & 5,988 & 6,604 & 6,919 \\
\hline Short-term foreign liabilities of the banking system 1 / & 4,916 & 4,597 & 4,389 & 4,504 & 4,517 \\
\hline Gross official reserves & 772 & 2,087 & 2,512 & 3,438 & 3,865 \\
\hline In months of imports GNFS & 3.7 & 9.2 & 8.2 & 8.9 & 8.1 \\
\hline In percent of short-term external debt excl. nonres FX deposits & 25.1 & 86.8 & 158.0 & 156.7 & 137.5 \\
\hline In percent of short-term external debt plus bank NR deposits & 14.3 & 45.1 & 61.6 & 76.6 & 74.2 \\
\hline In percent of short-term debt plus FX deposits & 7.0 & 20.0 & 27.7 & 33.1 & 34.4 \\
\hline In percent of broad money & 12.2 & 28.7 & 34.3 & 37.9 & 38.0 \\
\hline In percent of base money & 175.4 & 427.3 & 418.1 & 386.3 & 372.9 \\
\hline $\begin{array}{l}\text { Banking system foreign assets as percent of short-term external debt pl } \\
\text { all FX deposits } 2 / 3 \text { / }\end{array}$ & 46.6 & 56.9 & 68.1 & 74.5 & 71.5 \\
\hline \multicolumn{6}{|l|}{ Financial market indicators } \\
\hline Foreign currency debt rating (Moody's) 4/ & B3 & B3 & B3 & B3 & $\ldots$ \\
\hline Foreign currency debt rating (S\&P) 4/ & B- & B- & $\mathrm{B}$ & $\mathrm{B}+$ & $\ldots$ \\
\hline EMBI secondary market spread (bps, end of period) 4/ & 1,228 & 624 & 388 & 298 & 214 \\
\hline Exchange rate (per U.S. dollar, period average) & 21.6 & 28.2 & 28.6 & 24.4 & $\ldots$ \\
\hline Exchange rate (per U.S. dollar, end-of-period) & 27.2 & 29.3 & 26.4 & 24.1 & \\
\hline
\end{tabular}

Sources: Central Bank of Uruguay; and Fund staff estimates.

1/ Includes all use of Fund credit.

2/ By remaining maturity.

3/ Excludes nonreserve assets from the BCU.

4/ As of March 6, 2006. 
Table 7. Uruguay: Public Sector Financing Outlook 2006 1/

(In millions of U.S. dollars)

\begin{tabular}{|c|c|c|c|c|c|}
\hline & \multicolumn{5}{|c|}{ Projections } \\
\hline & Q1 & $\mathrm{Q} 2$ & Q3 & Q4 & Year \\
\hline Public sector gross borrowing requirements & 1,049 & 530 & 317 & 727 & 2,623 \\
\hline Of which: IMF repurchases & 48 & 113 & 245 & 271 & 678 \\
\hline Of which: World Bank/IDB 2/ & 556 & 77 & 43 & 65 & 741 \\
\hline Of which: Bond amortizations & 232 & 49 & 60 & 399 & 741 \\
\hline Gross financing & 1,049 & 530 & 317 & 727 & 2,623 \\
\hline Of which: IMF disbursements & 169 & 124 & 124 & 124 & 543 \\
\hline Of which: World Bank/IDB 2/ & 68 & 18 & 68 & 153 & 307 \\
\hline Of which: Bond placements & 762 & 313 & 131 & 510 & 1,716 \\
\hline 10 year maturity international issuances & 657 & 194 & 16 & 395 & 1,262 \\
\hline Of which: Prefinancing for 2007 & $\ldots$ & $\ldots$ & $\ldots$ & $\ldots$ & 400 \\
\hline UI bonds & 48 & 61 & 61 & 62 & 232 \\
\hline Letras & 56 & 59 & 54 & 54 & 222 \\
\hline Of which: Short-term debt & 0 & 38 & 19 & 0 & 92 \\
\hline \multicolumn{6}{|l|}{ Memorandum items: } \\
\hline Fund credit outstanding & $\ldots$ & $\ldots$ & $\ldots$ & $\ldots$ & 2,175 \\
\hline (as a share of quota) & $\ldots$ & $\ldots$ & $\ldots$ & $\ldots$ & 495 \\
\hline Net World Bank/IDB 2/ & -488 & -59 & 25 & 88 & -433 \\
\hline Net bond placements & 530 & 263 & 71 & 111 & 975 \\
\hline
\end{tabular}

Source: Central Bank of Uruguay; and Fund staff estimates.

1/ Excludes the assumption of net liabilities from BHU in 2006.

2/ Includes official debt. 
Table 8. Uruguay: Schedule of Purchases Under the Stand-By Arrangement, 2005-08

\begin{tabular}{|c|c|c|c|}
\hline \multirow[b]{2}{*}{ Availability Date } & \multicolumn{2}{|c|}{ Amount of Purchase } & \multirow[b]{2}{*}{ Conditions } \\
\hline & SDR & Percent Quota & \\
\hline June 8,2005 & 30.65 & 10.0 & Program approval \\
\hline September 28, 2005 & 30.65 & 10.0 & $\begin{array}{l}\text { Completion of first review and observance of structural PCs } \\
\text { and end-June quantitative PCs }\end{array}$ \\
\hline January 18, 2006 & 30.65 & 10.0 & $\begin{array}{l}\text { Completion of second review and observance of structural PCs } \\
\text { and end-December quantitative PCs } 1 /\end{array}$ \\
\hline March 27, 2006 & 85.82 & 28.0 & $\begin{array}{l}\text { Completion of third review and observance of structural PCs } \\
\text { and end-December quantitative PCs }\end{array}$ \\
\hline May 31, 2006 & 85.82 & 28.0 & $\begin{array}{l}\text { Completion of fourth review and observance of structural PCs } \\
\text { and end-March quantitative PCs }\end{array}$ \\
\hline August 31, 2006 & 85.82 & 28.0 & $\begin{array}{l}\text { Completion of fifth review and observance of structural PCs } \\
\text { and end-June quantitative PCs }\end{array}$ \\
\hline November 30, 2006 & 85.82 & 28.0 & $\begin{array}{l}\text { Completion of sixth review and observance of structural PCs } \\
\text { and end-September quantitative PCs }\end{array}$ \\
\hline February 28, 2007 & 67.43 & 22.0 & $\begin{array}{l}\text { Completion of seventh review and observance of structural PCs } \\
\text { and end-December quantitative PCs }\end{array}$ \\
\hline May 31, 2007 & 67.43 & 22.0 & $\begin{array}{l}\text { Completion of eighth review and observance of structural PCs } \\
\text { and end-March quantitative PCs }\end{array}$ \\
\hline August 31, 2007 & 67.43 & 22.0 & $\begin{array}{l}\text { Completion of ninth review and observance of structural PCs } \\
\text { and end-June quantitative PCs }\end{array}$ \\
\hline November 30, 2007 & 67.43 & 22.0 & $\begin{array}{l}\text { Completion of tenth review and observance of structural PCs } \\
\text { and end-September quantitative PCs }\end{array}$ \\
\hline February 28, 2008 & 30.65 & 10.0 & $\begin{array}{l}\text { Completion of eleventh review and observance of structural PCs } \\
\text { and end-December quantitative PCs }\end{array}$ \\
\hline May 31, 2008 & 30.65 & 10.0 & $\begin{array}{l}\text { Completion of twelfth review and observance of structural PCs } \\
\text { and end-March quantitative PCs }\end{array}$ \\
\hline Total access & 766.25 & & \\
\hline Percent of quota & 250.0 & & \\
\hline
\end{tabular}

Source: Fund staff estimates. 
Table 9. Uruguay: Projected Payments to the Fund and Capacity to Repay

\begin{tabular}{|c|c|c|c|c|c|c|c|c|}
\hline & 2003 & 2004 & 2005 & 2006 & 2007 & 2008 & 2009 & 2010 \\
\hline Fund disbursements (existing and prospective) & \multicolumn{8}{|c|}{ (In SDR million) } \\
\hline Principal disbursements & 364.2 & 372.8 & 201.1 & 373.9 & 269.7 & 61.3 & 0.0 & 0.0 \\
\hline Existing & 364.2 & 372.8 & 201.1 & 30.7 & 0.0 & 0.0 & 0.0 & 0.0 \\
\hline Prospective & 0.0 & 0.0 & 0.0 & 343.3 & 269.7 & 61.3 & 0.0 & 0.0 \\
\hline \multicolumn{9}{|c|}{ Fund repurchases and charges (existing and prospective) } \\
\hline Principal (repurchases) 1/ & 57.1 & 270.3 & 317.3 & 467.9 & 540.9 & 332.3 & 310.4 & 274.2 \\
\hline On existing purchases & 57.1 & 270.3 & 317.3 & 467.9 & 540.9 & 332.3 & 246.0 & 52.0 \\
\hline On prospective purchases & 0.0 & 0.0 & 0.0 & 0.0 & 0.0 & 0.0 & 64.4 & 222.2 \\
\hline Charges and interest $2 /$ & 45.9 & 64.0 & 77.9 & 91.2 & 78.3 & 62.1 & 44.2 & 27.7 \\
\hline On existing purchases & 45.9 & 64.0 & 77.9 & 86.0 & 49.9 & 24.7 & 10.9 & 2.4 \\
\hline On prospective purchases & 0.0 & 0.0 & 0.0 & 5.1 & 28.4 & 37.3 & 33.3 & 25.4 \\
\hline Total payments to Fund 1/ & 103.0 & 334.4 & 395.2 & 559.0 & 619.2 & 394.3 & 354.6 & 301.9 \\
\hline In millions of U.S. dollars & 144.3 & 495.2 & 584.0 & 800.8 & 887.7 & 565.8 & 508.8 & 432.9 \\
\hline In percent of exports of goods and NFS & 4.7 & 12.4 & 12.4 & 15.8 & 16.2 & 9.5 & 8.0 & 6.4 \\
\hline In percent of GDP & 1.3 & 3.7 & 3.5 & 4.2 & 4.4 & 2.7 & 2.3 & 1.8 \\
\hline In percent of quota & 33.6 & 109.1 & 128.9 & 182.4 & 202.0 & 128.7 & 115.7 & 98.5 \\
\hline In percent of overall external debt service & 8.9 & 30.2 & 24.4 & 26.2 & 35.7 & 25.4 & 23.2 & 17.5 \\
\hline In percent of gross reserves & 6.9 & 19.7 & 17.0 & 20.7 & 22.1 & 13.6 & 11.6 & 9.5 \\
\hline Fund credit outstanding & \multicolumn{8}{|c|}{ (End-of-period) } \\
\hline In millions of SDRs & $1,625.9$ & $1,728.4$ & $1,612.2$ & $1,518.2$ & $1,247.1$ & 976.1 & 665.7 & 391.6 \\
\hline In millions of U.S. dollars & $2,385.9$ & $2,662.3$ & $2,307.5$ & $2,175.7$ & $1,788.6$ & $1,400.7$ & 955.0 & 561.3 \\
\hline In percent of exports of goods and NFS & 77.4 & 66.4 & 49.0 & 42.9 & 32.6 & 23.6 & 15.0 & 8.3 \\
\hline In percent of GDP & 21.3 & 20.1 & 13.8 & 11.5 & 8.9 & 6.6 & 4.3 & 2.4 \\
\hline In percent of quota & 530.5 & 563.9 & 526.0 & 495.3 & 406.9 & 318.5 & 217.2 & 127.8 \\
\hline In percent of public sector external debt & 25.0 & 26.1 & 22.6 & 21.0 & 17.4 & 13.8 & 9.5 & 5.7 \\
\hline In percent of overall external debt & 21.7 & 23.0 & 20.2 & 18.8 & 15.5 & 12.3 & 8.5 & 5.0 \\
\hline In percent of gross reserves & 114.3 & 106.0 & 67.1 & 56.3 & 44.6 & 33.6 & 21.8 & 12.3 \\
\hline Memorandum items: & \multicolumn{8}{|c|}{ (In millions of U.S. dollars unless otherwise noted) } \\
\hline Exports of goods and NFS & 3,084 & 4,008 & 4,705 & 5,076 & 5,480 & 5,926 & 6,349 & 6,802 \\
\hline Quota (millions of SDRs) & 306.5 & 306.5 & 306.5 & 306.5 & 306.5 & 306.5 & 306.5 & 306.5 \\
\hline GDP & 11,211 & 13,267 & 16,724 & 18,913 & 20,081 & 21,208 & 22,252 & 23,424 \\
\hline U.S. dollar per SDR, e.o.p. & 1.47 & 1.54 & 1.43 & 1.43 & 1.43 & 1.43 & 1.43 & 1.43 \\
\hline U.S. dollar per SDR, average & 1.40 & 1.48 & 1.48 & 1.43 & 1.43 & 1.43 & 1.43 & 1.43 \\
\hline Public sector debt & 11,705 & 12,272 & 11,696 & 12,354 & 12,455 & 12,532 & 12,585 & 12,649 \\
\hline Public sector external debt $3 /$ & 9,557 & 10,206 & 10,225 & 10,344 & 10,291 & 10,166 & 10,065 & 9,900 \\
\hline Overall external debt service & 1,614 & 1,638 & 2,397 & 3,062 & 2,490 & 2,228 & 2,189 & 2,481 \\
\hline Overall external debt & 11,012 & 11,594 & 11,447 & 11,566 & 11,513 & 11,388 & 11,287 & 11,122 \\
\hline Gross foreign reserves & 2,087 & 2,512 & 3,438 & 3,865 & 4,015 & 4,165 & 4,375 & 4,576 \\
\hline
\end{tabular}

Sources: Finance Department; and Fund staff estimates and projections.

1/ Projections on obligations basis.

2/ Includes charges for GRA credit outstanding and other charges (for example, SDR charges). Projections are based on the end-Jaunary 2006 basic rate of charge in the GRA of 4.37 percent plus burden-sharing of 18 basis points.

3/ Excluding public commercial banks. 
Table 1. Uruguay: Public Sector Debt Sustainability 1/

\begin{tabular}{|c|c|c|c|c|c|c|c|c|c|c|c|c|}
\hline & \multicolumn{7}{|c|}{ Est. } & \multicolumn{3}{|c|}{ Projections } & \multirow[b]{2}{*}{2011} & \multirow[b]{2}{*}{2012} \\
\hline & 2001 & 2002 & 2003 & 2004 & $2005^{-}$ & 2006 & 2007 & 2008 & 2009 & 2010 & & \\
\hline \multicolumn{13}{|c|}{ A. Assumptions } \\
\hline Real GDP growth (percent) & -3.4 & -11.0 & 2.2 & 12.3 & 6.0 & 4.0 & 3.5 & 3.0 & 3.0 & 3.0 & 3.0 & 3.0 \\
\hline Interest rate spread (bps) & 284 & 1400 & 900 & 600 & 387 & 300 & 300 & 300 & 300 & 300 & 300 & 300 \\
\hline Real U.S. dollar exchange rate (avg.) (change in percent) & -13.7 & -11.0 & -22.1 & -0.2 & 12.6 & 6.9 & 1.0 & 0.7 & 0.2 & 0.0 & 0.0 & 0.0 \\
\hline Primary balance: Consolidated public sector & -1.2 & 0.0 & 2.7 & 3.8 & 3.8 & 3.7 & 4.0 & 4.0 & 4.0 & 4.0 & 4.0 & 4.0 \\
\hline Non-financial public sector & -1.0 & 0.2 & 2.9 & 4.0 & 4.0 & 3.9 & 4.2 & 4.2 & 4.2 & 4.2 & 4.2 & 4.2 \\
\hline BCU 2/ & -0.2 & -0.2 & -0.2 & -0.2 & -0.2 & -0.2 & -0.2 & -0.2 & -0.2 & -0.2 & -0.2 & -0.2 \\
\hline Overall Balance: Consolidated public sector & -4.1 & -4.6 & -3.2 & -2.2 & -0.8 & -1.1 & -0.6 & -0.5 & -0.4 & -0.4 & -0.4 & -0.4 \\
\hline Non-financial public sector & -3.8 & -4.3 & -2.9 & -1.2 & -0.6 & -0.8 & -0.3 & -0.2 & -0.1 & -0.1 & -0.1 & -0.1 \\
\hline $\mathrm{BCU} 2 /$ & -0.3 & -0.3 & -0.4 & -1.0 & -0.2 & -0.3 & -0.3 & -0.3 & -0.3 & -0.3 & -0.3 & -0.3 \\
\hline \multicolumn{13}{|c|}{ B. Debt Dynamics (in percent of GDP) } \\
\hline $\begin{array}{l}\text { Gross non-financial public sector debt } \\
\text { of which: }\end{array}$ & 46 & 96 & 104 & 92 & 70 & 65 & 62 & 59 & 57 & 54 & 51 & 49 \\
\hline Contribution from REER (cum since 2005) & $\ldots$ & $\ldots$ & $\ldots$ & $\ldots$ & 0 & 4 & 5 & 5 & 5 & 5 & 5 & 4 \\
\hline Contribution from Growth (cum since 2005) & $\ldots$ & $\ldots$ & $\ldots$ & $\ldots$ & 0 & 4 & 8 & 11 & 14 & 17 & 20 & 23 \\
\hline \multicolumn{13}{|c|}{ C. Cash Flow (in millions of U.S. dollars) } \\
\hline Gross borrowing needs & 1210 & 3209 & 1251 & 2504 & 2588 & 2973 & 1777 & 1504 & 1496 & 1802 & 2082 & 1388 \\
\hline Augmented public sector deficit & 707 & 2524 & 324 & 161 & 95 & 569 & 65 & 45 & 27 & 42 & 30 & 40 \\
\hline Public sector deficit & 707 & 526 & 324 & 161 & 95 & 149 & 55 & 36 & 17 & 34 & 23 & 34 \\
\hline Bank assistance 3/ & 0 & 1998 & 0 & 0 & 0 & 420 & 10 & 10 & 10 & 8 & 7 & 6 \\
\hline Buildup in free reserves at the central bank & -7 & -364 & 6 & 631 & 633 & 100 & 100 & 100 & 100 & 100 & 100 & 100 \\
\hline Amortization & 510 & 1049 & 921 & 1711 & 1859 & 2303 & 1611 & 1359 & 1369 & 1660 & 1952 & 1248 \\
\hline Long-term bonds and inflation-indexed bonds & 403 & 349 & 221 & 29 & 289 & 631 & 129 & 186 & 184 & 488 & 836 & 348 \\
\hline Letras (2 years) & $\ldots$ & $\ldots$ & $\ldots$ & $\ldots$ & 422 & 110 & 280 & 222 & 250 & 250 & 250 & 250 \\
\hline Peso bonds (short-term) & 0 & 209 & 209 & 637 & 127 & 45 & 57 & 50 & 50 & 50 & 50 & 50 \\
\hline Commercial bank loans & 0 & 214 & 217 & 307 & 89 & 69 & 99 & 114 & 128 & 149 & 90 & 94 \\
\hline Supplier credits & 0 & 0 & 0 & 0 & 42 & 30 & 31 & 33 & 28 & 31 & 28 & 24 \\
\hline IDB/WB and other official debt & 97 & 205 & 234 & 339 & 411 & 741 & 240 & 276 & 284 & 298 & 317 & 317 \\
\hline IMF & 10 & 72 & 40 & 400 & 480 & 678 & 775 & 477 & 445 & 393 & 380 & 165 \\
\hline Gross Financing 3/ & 1209 & 3209 & 1251 & 2504 & 2588 & 2973 & 1777 & 1504 & 1496 & 1802 & 2082 & 1388 \\
\hline Long-term bonds (external) & 1292 & 143 & 405 & 371 & 1091 & 1262 & 242 & 454 & 507 & 809 & 1091 & 411 \\
\hline Inflation-indexed instruments & $\ldots$ & $\ldots$ & $\ldots$ & $\ldots$ & 318 & 232 & 250 & 250 & 250 & 250 & 250 & 250 \\
\hline Letras ( 2 years) & $\ldots$ & $\ldots$ & $\ldots$ & $\ldots$ & 310 & 222 & 250 & 250 & 250 & 250 & 250 & 250 \\
\hline Peso bonds (short-term) & -15 & 677 & 637 & 544 & 92 & 57 & 50 & 50 & 50 & 50 & 50 & 50 \\
\hline Commercial bank & 33 & -16 & -475 & 365 & 19 & -55 & 99 & 114 & 128 & 149 & 90 & 94 \\
\hline Use of deposits & 26 & -21 & -543 & 200 & -103 & 124 & 0 & 0 & 0 & 0 & 0 & 0 \\
\hline Loans & 7 & 5 & 68 & 165 & 122 & 69 & 99 & 114 & 128 & 149 & 90 & 94 \\
\hline IDB/WB and other official debt & 198 & 789 & 461 & 366 & 370 & 307 & 468 & 265 & 284 & 263 & 323 & 309 \\
\hline IMF & 0 & 1603 & 484 & 552 & 297 & 543 & 387 & 88 & 0 & 0 & 0 & 0 \\
\hline Central bank credit (net) (net of IMF disbursements) & 0 & 0 & 0 & 0 & 0 & 0 & 0 & 0 & 0 & 0 & 0 & 0 \\
\hline Other inflows (net) $4 /$ & -299 & 13 & -261 & 307 & 91 & 404 & 31 & 33 & 28 & 31 & 28 & 24 \\
\hline Residual financing needs & $\mathbf{0}$ & $\mathbf{0}$ & $\mathbf{0}$ & $\mathbf{0}$ & $\mathbf{0}$ & $\mathbf{0}$ & $\mathbf{0}$ & $\mathbf{0}$ & $\mathbf{0}$ & $\mathbf{0}$ & $\mathbf{0}$ & $\mathbf{0}$ \\
\hline \multicolumn{13}{|c|}{ D. Other Indicators } \\
\hline Total debt service (in percent of GDP) & 5.6 & 13.1 & 14.0 & 18.1 & 15.6 & 16.9 & 12.6 & 10.9 & 10.5 & 11.5 & 12.2 & 9.2 \\
\hline Average interest rate (in percent) & 12.6 & 5.5 & 5.7 & 6.6 & 6.3 & 7.5 & 7.4 & 7.6 & 7.7 & 8.2 & 8.5 & 9.0 \\
\hline \multicolumn{13}{|l|}{ Memorandum items: } \\
\hline GDP (millions of dollars) & 18,561 & 12,089 & 11,211 & 13,267 & 16,724 & 18,913 & 20,081 & 21,208 & 22,252 & 23,424 & 24,759 & 26,071 \\
\hline Nominal debt (millions of dollars) & 8,538 & 11,551 & 11,705 & 12,272 & 11,696 & 12,354 & 12,455 & 12,532 & 12,585 & 12,649 & 12,702 & 12,765 \\
\hline Commerical bond placements (net) (millions of dollars) & 889 & -207 & 184 & 342 & 1,008 & 975 & 333 & 545 & 573 & 571 & 505 & 312 \\
\hline World Bank/IDB (net) & $\ldots$ & $\ldots$ & $\ldots$ & $\ldots$ & 49 & -398 & 240 & -3 & 6 & -29 & 12 & -3 \\
\hline Rollover of Fund obligations (in percent) & $\ldots$ & $\ldots$ & $\ldots$ & $\ldots$ & 62 & 80 & 50 & 18 & 0 & 0 & 0 & 0 \\
\hline Fund credit outstanding (millions of dollars) & 145 & 1,754 & 2,386 & 2,662 & 2,308 & 2,175 & 1,788 & 1,401 & 955 & 562 & 181 & 16 \\
\hline Fund credit outstanding (in percent of GDP) & 0.8 & 14.5 & 21.3 & 20.1 & 13.8 & 11.5 & 8.9 & 6.6 & 4.3 & 2.4 & 0.7 & 0.1 \\
\hline Fund credit outstanding (as a share of quota) & 0.5 & 5.5 & 530.5 & 563.9 & 526.0 & 495.3 & 406.9 & 318.5 & 217.2 & 127.8 & 41.3 & 3.8 \\
\hline
\end{tabular}

Sources: Ministry of Finance, Banco Central del Uruguay, and Fund staff estimates.

1/ Net of free reserves and monetary policy instruments.

2/ This does not reflect the planned strengthening of the BCU finances (SB for August 2006) yet.

The overall fiscal position will not be affected as interest costs of the central bank would be shifted to the government.

3/ Includes in 2006 the funding of the deposit insurance scheme of US\$60 million and assumption of the BHU liabilities of US\$350 million (net),

of which US\$990 million are in form of liabilities and US\$640 in form of financial assets (assumed recovery rate of 64 percent).

4/ In 2006: assumption of BHU financial obligations and cancellation of BHU deposits. 
Table 2. Uruguay: Public Sector Debt Sustainability Framework, 2001-2011 (In percent of GDP, unless otherwise indicated)

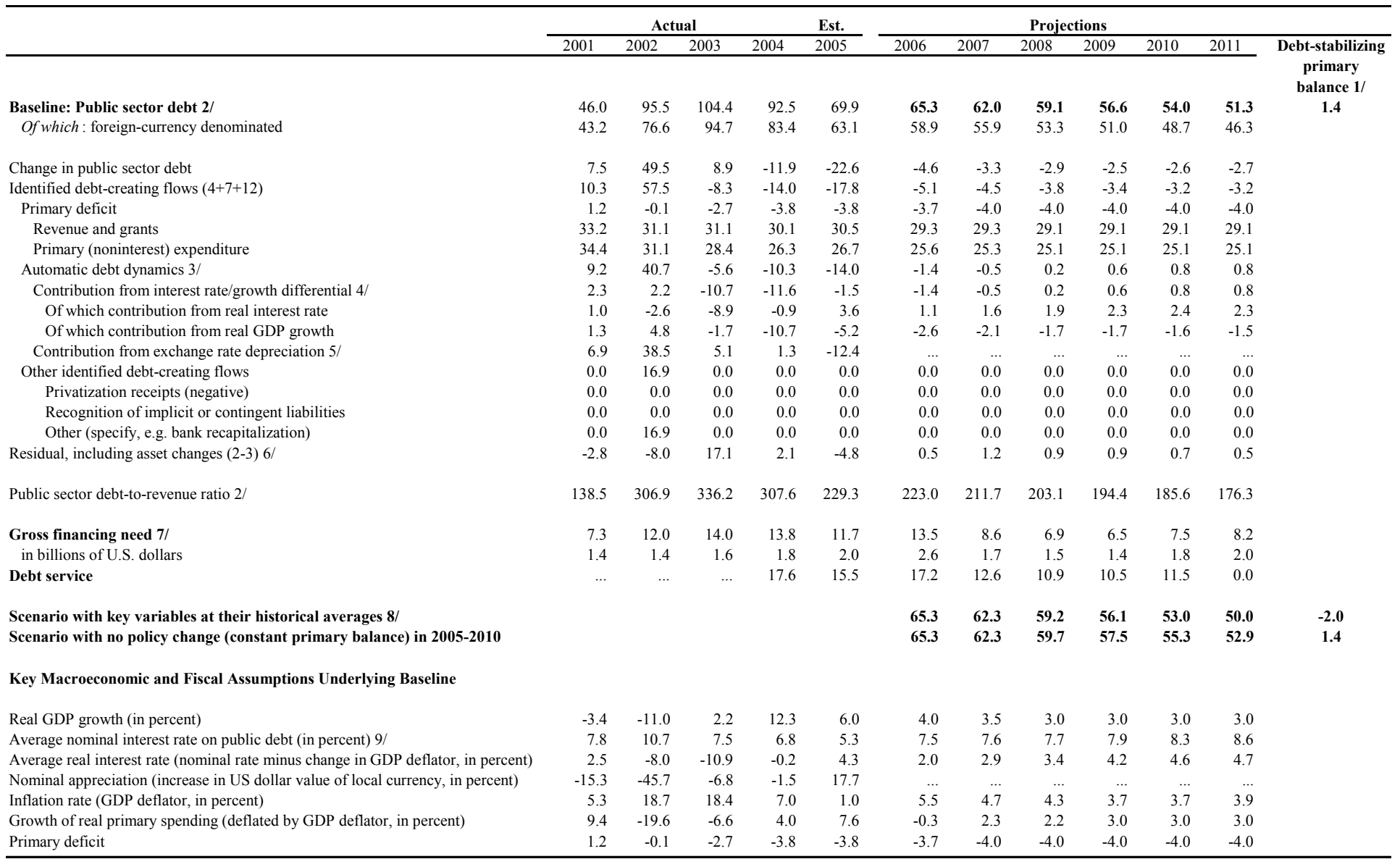

$1 /$ Assumes that key variables (real GDP growth, real interest rate, and other identified debt-creating flows) remain at the level of the last projection year.

2/ Framework covers the public sector (net of free reserves and monetary policy instruments).

$3 /$ Derived as $[(r-p(1+g)-g+a e(1+r)] /(1+g+p+g p))$ times previous period debt ratio, with $r=$ interest rate; $p=$ growth rate of GDP deflator; $g=$ real GDP growth rate; $a=$ share of foreign-currency

denominated debt; and $\mathrm{e}=$ nominal exchange rate depreciation (measured by increase in local currency value of U.S. dollar).

4/ The real interest rate contribution is derived from the denominator in footnote $3 /$ as $r-p(1+g)$ and the real growth contribution as $-\mathrm{g}$

$5 /$ The exchange rate contribution is derived from the numerator in footnote $3 /$ as ae $(1+\mathrm{r})$.

6/ For projections, this line includes exchange rate changes.

作

8/ The key variables include real GDP growh, real interest rate, and primary balance in percent of GDP.

9/ Derived as nominal interest expenditure divided by previous period debt stock. 
Figure 1. Uruguay: Public Debt Sustainability: Bound Tests 1/

(Public debt in percent of GDP)

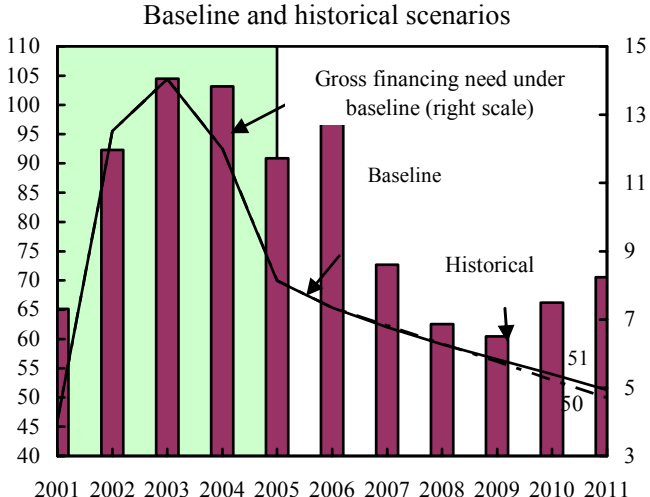

Growth shock (in percent per year)

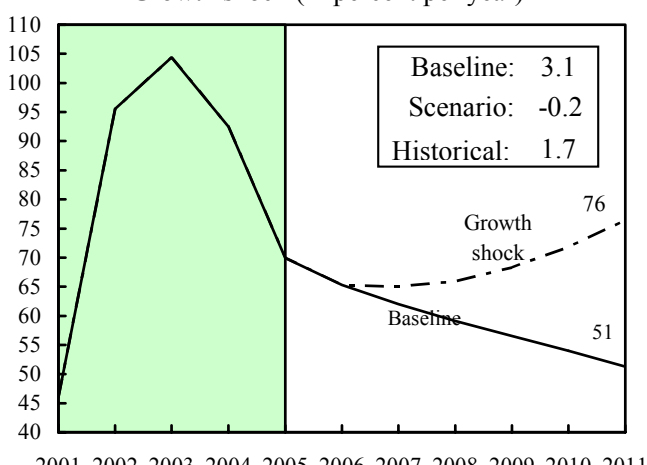

Combined shock 2/

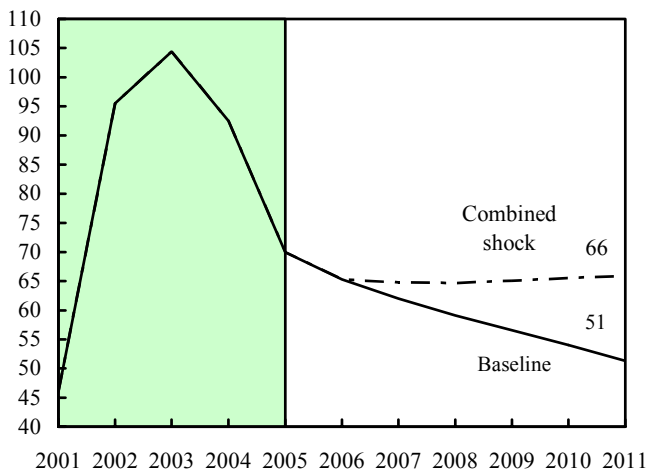

Interest rate shock (in percent)

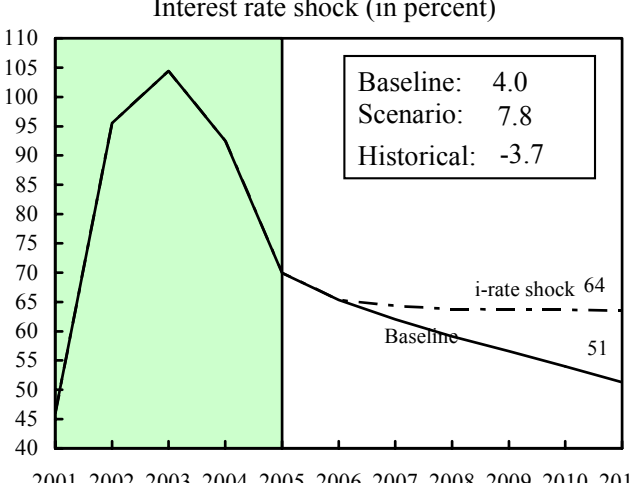

Primary balance shock (in percent of GDP) and no policy change scenario (constant primary balance

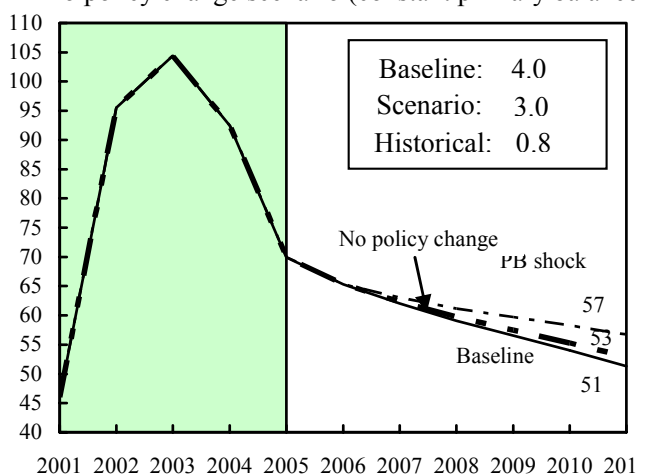

Real depreciation and contingent liabilities shocks 3/

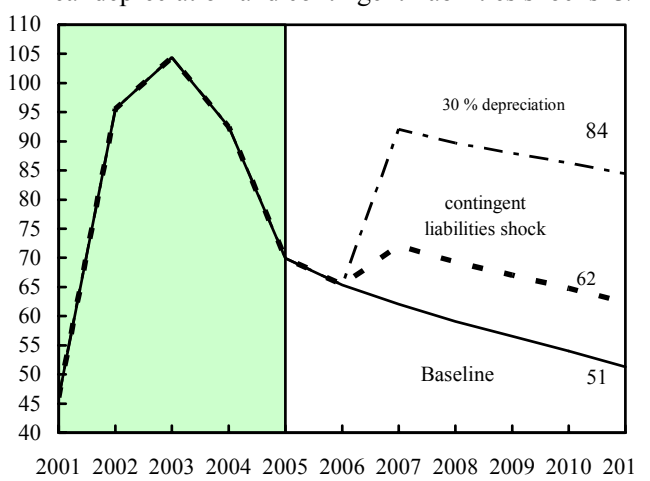

Sources: International Monetary Fund, Country desk data, and staff estimates.

1/ Shaded areas represent actual data.Individual shocks are permanent one-half standard deviation shocks.

Figures in the boxes represent average projections for the respective variables in the baseline and scenario bein! presented. Ten-year historical average for the variable is also shown.

2/ Permanent $1 / 4$ standard deviation shocks applied to real interest rate, growth rate, and primary balance.

3/ One-time real depreciation of 30 percent and 10 percent of GDP shock to contingent liabilities occur in 2006 with real depreciation defined as nominal depreciation (measured by percentage fall in dollar value of local currency) minus domestic inflation (based on GDP deflator). 
Table 3. Uruguay: External Debt Sustainability Framework, 2001-2011

(In percent of GDP, unless otherwise indicated)

\begin{tabular}{|c|c|c|c|c|c|c|c|c|c|c|c|c|}
\hline & \multicolumn{4}{|c|}{ Actual } & \multirow{2}{*}{$\begin{array}{c}\text { Est. } \\
2005 \\
\end{array}$} & \multicolumn{7}{|c|}{ Projections } \\
\hline & 2001 & 2002 & 2003 & 2004 & & 2006 & 2007 & 2008 & 2009 & 2010 & 2011 & Debt-stabilizing \\
\hline Baseline: External debt & 48.1 & 87.5 & 98.2 & 87.4 & 68.4 & 61.2 & 57.3 & 53.7 & 50.7 & 47.5 & 44.8 & $\begin{array}{c}\text { current account 6/ } \\
-1.1\end{array}$ \\
\hline Change in external debt & 3.9 & 39.4 & 10.7 & -10.8 & -18.9 & -7.3 & -3.8 & -3.6 & -3.0 & -3.2 & -2.7 & \\
\hline Identified external debt-creating flows $(4+8+9)$ & 4.8 & 21.1 & 3.8 & -16.9 & -19.7 & -3.0 & -2.6 & -2.5 & -2.3 & -2.5 & -2.2 & \\
\hline Current account deficit, excluding interest payments & 0.2 & -7.8 & -2.6 & -4.4 & -2.6 & 0.6 & -1.2 & -1.5 & -2.5 & -2.5 & -2.2 & \\
\hline Deficit in balance of goods and services & 2.4 & -1.7 & -3.1 & -2.5 & -0.3 & 3.3 & 1.5 & 1.0 & -0.2 & -0.2 & 0.0 & \\
\hline Exports & 17.6 & 22.3 & 27.5 & 30.2 & 28.1 & 26.8 & 27.3 & 27.9 & 28.5 & 29.0 & 29.4 & \\
\hline Imports & 20.1 & 20.6 & 24.4 & 27.7 & 27.9 & 30.1 & 28.7 & 29.0 & 28.4 & 28.8 & 29.4 & \\
\hline Net non-debt creating capital inflows (negative) & -1.7 & -1.5 & -3.6 & -2.5 & -3.7 & -6.2 & -4.2 & -4.0 & -2.7 & -2.8 & -2.8 & \\
\hline A1. Key variables are at their historical averages in 2007-11 3/ & 6.3 & 30.4 & 9.9 & -9.9 & -13.4 & 2.6 & 2.8 & 2.9 & 2.9 & 2.8 & 2.8 & \\
\hline Contribution from nominal interest rate & 2.7 & 4.6 & 3.1 & 5.2 & 4.7 & 5.0 & 4.8 & 4.5 & 4.4 & 4.3 & 4.1 & \\
\hline Contribution from real GDP growth & 1.6 & 8.2 & -2.1 & -10.2 & -4.2 & -2.4 & -2.0 & -1.6 & -1.5 & -1.4 & -1.4 & \\
\hline Contribution from price and exchange rate changes $2 /$ & 2.0 & 17.6 & 8.9 & -4.9 & -13.9 & $\ldots$ & $\ldots$ & $\ldots$ & $\ldots$ & $\ldots$ & $\ldots$ & \\
\hline Residual, incl. change in gross foreign assets (2-3) $3 /$ & -0.9 & 18.2 & 6.9 & 6.1 & 0.8 & -4.3 & -1.2 & -1.1 & -0.6 & -0.8 & -0.5 & \\
\hline External debt-to-exports ratio (in percent) & 272.8 & 392.8 & 357.1 & 289.3 & 243.3 & 227.8 & 210.1 & 192.2 & 177.8 & 163.5 & 152.2 & \\
\hline Gross external financing need (in billions of US dollars) 4 / & 3.5 & 3.8 & 3.0 & 2.0 & 2.7 & 3.4 & 2.6 & 2.1 & 1.8 & 2.0 & 2.2 & \\
\hline B5. One time 30 percent nominal depreciation in 2007 & 19.0 & 31.3 & 26.4 & 14.8 & 16.3 & 18.1 & 12.7 & 9.8 & 8.0 & 8.7 & 9.0 & \\
\hline Scenario with key variables at their historical averages 5/ & & & & & & 61.2 & 61.7 & 62.4 & 63.2 & 64.0 & 65.2 & 2.2 \\
\hline \multicolumn{13}{|l|}{ Key Macroeconomic Assumptions Underlying Baseline } \\
\hline Real GDP growth (in percent) & -3.4 & -11.0 & 2.2 & 12.3 & 6.0 & 4.0 & 3.5 & 3.0 & 3.0 & 3.0 & 3.0 & \\
\hline GDP deflator in US dollars (change in percent) & -4.4 & -26.8 & -9.2 & 5.2 & 18.9 & 8.7 & 2.6 & 2.5 & 1.9 & 2.2 & 2.2 & \\
\hline Nominal external interest rate (in percent) & 5.6 & 6.2 & 3.2 & 6.2 & 6.8 & 8.3 & 8.3 & 8.4 & 8.6 & 8.8 & 9.2 & \\
\hline Growth of exports (US dollar terms, in percent) & -10.5 & -17.8 & 14.5 & 30.0 & 17.4 & 7.9 & 7.9 & 8.1 & 7.1 & 7.1 & 6.7 & \\
\hline Growth of imports (US dollar terms, in percent) & -11.2 & -33.1 & 9.7 & 34.3 & 26.9 & 22.3 & 1.3 & 6.5 & 2.7 & 7.0 & 7.3 & \\
\hline Current account balance, excluding interest payments & -0.2 & 7.8 & 2.6 & 4.4 & 2.6 & -0.6 & 1.2 & 1.5 & 2.5 & 2.5 & 2.2 & \\
\hline Net non-debt creating capital inflows & 1.7 & 1.5 & 3.6 & 2.5 & 3.7 & 6.2 & 4.2 & 4.0 & 2.7 & 2.8 & 2.8 & \\
\hline
\end{tabular}

Net non-debt creating capital inflows

1/ Derived as $[\mathrm{r}-\mathrm{g}-\rho(1+\mathrm{g})+\varepsilon \alpha(1+\mathrm{r})] /(1+\mathrm{g}+\rho+\mathrm{g} \rho)$ times previous period debt stock, with $\mathrm{r}=$ nominal effective interest rate on external debt; $\rho=$ change in domestic GDP deflator in US dollar terms, $\mathrm{g}=$ real GDP $\gamma \rho \circ \omega \tau \eta ~ \rho \alpha \tau \varepsilon, \varepsilon=$ nominal appreciation (increase in dollar value of domestic currency), and $\alpha=$ share of domestic-currency denominated debt in total external debt.

$2 /$ The contribution from price and exchange rate changes is defined as $[-\rho(1+\mathrm{g})+\varepsilon \alpha(1+\mathrm{r})](1+\mathrm{g}+\rho+\mathrm{g} \rho)$ times previous period debt stock. $\rho$ increases with an appreciating domestic currency $(\varepsilon>0)$ and rising inflation (based on GDP deflator).

3/ For projection, line includes the impact of price and exchange rate changes.

4/ Defined as current account deficit, plus amortization on medium- and long-term debt, plus short-term debt at end of previous period.

5/ The key variables include real GDP growth; nominal interest rate; dollar deflator growth; and both non-interest current account and non-debt inflows in percent of GDP.

6/ Long-run, constant balance that stabilizes the debt ratio assuming that key variables (real GDP growth, nominal interest rate, dollar deflator growth, and non-debt inflows in percent of GDP) remain at their levels

of the last projection year. 
Figure 2. Uruguay: External Debt Sustainability: Bound Tests 1/

(External debt in percent of GDP)
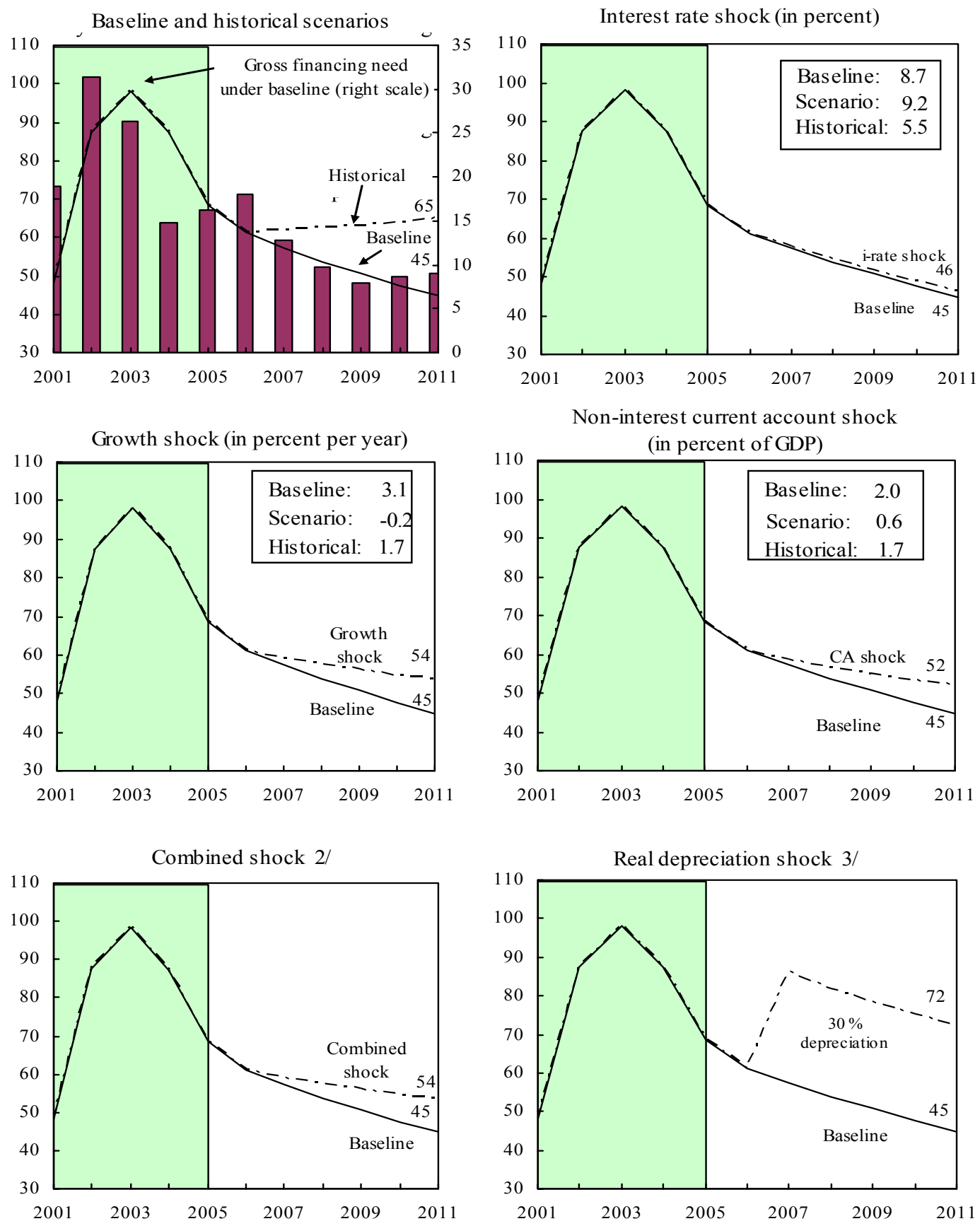

Sources: International Monetary Fund, Country desk data, and staff estimates.

1/ Shaded areas represent actual data. Individual shocks are permanent one-half standard deviation shocks. Figures in the boxes represent average projections for the respective variables in the baseline and scenario being presented. Ten-year historical average for the variable is also shown. 2/ Permanent 1/4 standard deviation shocks applied to real interest rate, growth rate, and current account balance.

3/ One-time real depreciation of 30 percent occurs in 2007. 
Table 4. Uruguay: Public Sector Debt Sustainability Framework—Gross Public Sector Financing Need, 2001-2011

(In percent of GDP, unless otherwise indicated)

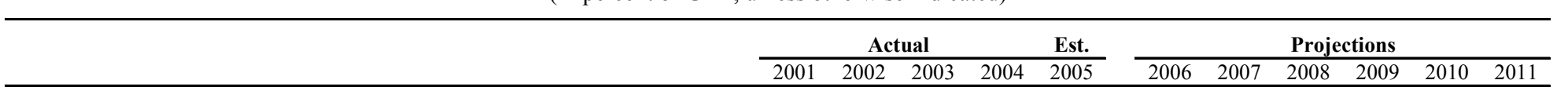

I. Baseline Projections

Gross financing need $1 /$

in billions of U.S. dollars

$\begin{array}{rrrrrrrrrrr}7.3 & 12.0 & 14.0 & 13.8 & 11.7 & 13.5 & 8.6 & 6.9 & 6.5 & 7.5 & 8.2 \\ 1.4 & 1.4 & 1.6 & 1.8 & 2.0 & 2.6 & 1.7 & 1.5 & 1.4 & 1.8 & 2.0\end{array}$

II. Stress Tests

Gross financing need 2/

$\begin{array}{rrrrrr}13.5 & 7.9 & 6.0 & 5.3 & 6.2 & 7.0 \\ 13.5 & 8.9 & 7.3 & 7.0 & 8.0 & 8.9 \\ & & & & & \\ 13.5 & 11.2 & 9.8 & 9.9 & 11.6 & 13.1 \\ 13.5 & 10.0 & 9.6 & 11.0 & 14.4 & 18.1 \\ 13.5 & 9.8 & 8.2 & 8.0 & 9.3 & 10.4 \\ 13.5 & 10.7 & 9.4 & 9.6 & 11.4 & 13.2 \\ 13.5 & 12.4 & 12.3 & 12.2 & 14.2 & 15.9 \\ 13.5 & 9.8 & 8.7 & 8.4 & 9.7 & 10.8\end{array}$

A1. Key variables are at their historical averages in 2006-10 3/

A2. No policy change (constant primary balance) in 2006-10

\section{B. Bound Tests}

B1. Real interest rate is at baseline plus one-half standard deviations

B2. Real GDP growth is at baseline minus one-half standard deviations

B3. Primary balance is at baseline minus one-half standard deviations

B4. Combination of B1-B3 using 1/4 standard deviation shocks

B5. One time 30 percent real depreciation in 20064

B6. 10 percent of GDP increase in other debt-creating flows in 2006

\section{Gross financing need in billions of U.S. dollars 2/}

\section{A. Alternative Scenarios}

A1. Key variables are at their historical averages in 2006-10 3/

A2. No policy change (constant primary balance) in 2006-10

$\begin{array}{llllll}2.6 & 1.6 & 1.2 & 1.1 & 1.4 & 1.6 \\ 2.6 & 1.8 & 1.5 & 1.5 & 1.9 & 2.2 \\ & & & & & \\ 2.6 & 2.2 & 2.1 & 2.2 & 2.7 & 3.3 \\ 2.6 & 1.9 & 1.9 & 2.2 & 3.0 & 3.8 \\ 2.6 & 2.0 & 1.7 & 1.8 & 2.2 & 2.6 \\ 2.6 & 2.1 & 1.9 & 2.0 & 2.5 & 3.0 \\ 2.6 & 1.7 & 1.7 & 1.8 & 2.2 & 2.6 \\ 2.6 & 2.0 & 1.8 & 1.9 & 2.3 & 2.7\end{array}$

B1. Real interest rate is at baseline plus one-half standard deviations

B2. Real GDP growth is at baseline minus one-half standard deviations

B3. Primary balance is at baseline minus one-half standard deviations

B4. Combination of B1-B3 using 1/4 standard deviation shocks

B5. One time 30 percent real depreciation in 20064

B6. 10 percent of GDP increase in other debt-creating flows in 2006

1/ Defined as public sector deficit, plus amortization of medium and long-term public sector debt, plus short-term debt at end of previous period. Excludes assumption of BHU liabilities for 2006.

2/ Gross financing under the stress test scenarios is derived by assuming the same ratio of short-term to total debt as in the baseline scenario and the same average maturity on medium- and long-term debt. Interest expenditures are derived by applying the respective interest rate to the previous period debt stock under each alternative scenario. 3/ The key variables include real GDP growth; real interest rate; and primary balance in percent of GDP.

4/ Real depreciation is defined as nominal depreciation (measured by percentage fall in dollar value of local currency) minus domestic inflation (based on GDP deflator). 
Table 5. Uruguay: External Sustainability Framework--Gross External Financing Need, 2001-2011

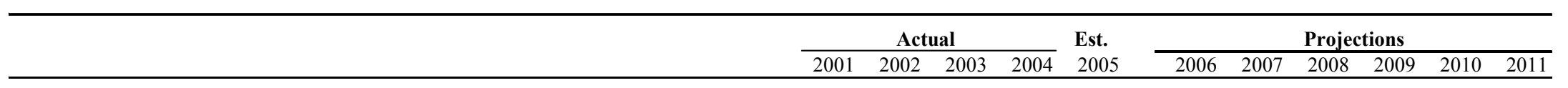

I. Baseline Projections

\section{Gross external financing need in billions of U.S. dollars 1/} in percent of GDP

$\begin{array}{rrrrrrrrrrr}3.5 & 3.8 & 3.0 & 2.0 & 2.7 & 3.4 & 2.6 & 2.1 & 1.8 & 2.0 & 2.2 \\ 19.0 & 31.3 & 26.4 & 14.8 & 16.3 & 18.1 & 12.7 & 9.8 & 8.0 & 8.7 & 9.0\end{array}$

II. Stress Tests

Gross external financing need in billions of U.S. dollars $2 /$

$\begin{array}{llllll}3.4 & 2.2 & 1.8 & 1.7 & 2.0 & 2.2 \\ & & & & & \\ 3.4 & 2.6 & 2.2 & 1.9 & 2.2 & 2.4 \\ 3.4 & 2.6 & 2.1 & 1.8 & 2.1 & 2.4 \\ 3.4 & 2.9 & 2.4 & 2.2 & 2.6 & 3.0 \\ 3.4 & 2.7 & 2.3 & 2.1 & 2.4 & 2.7 \\ 3.4 & 2.6 & 2.2 & 2.0 & 2.4 & 2.6\end{array}$

\section{A. Alternative Scenarios}

A1. Key variables are at their historical averages in 2007-11 3/

\section{B. Bound Tests}

B1. Nominal interest rate is at baseline plus one-half standard deviations

B2. Real GDP growth is at baseline minus one-half standard deviations

B3. Non-interest current account is at baseline minus one-half standard deviations

B4. Combination of B1-B3 using 1/4 standard deviation shocks

B5. One time 30 percent nominal depreciation in 2007

Gross external financing need in percent of GDP 2/

\section{A. Alternative Scenarios}

A1. Key variables are at their historical averages in 2007-11 3/

\begin{tabular}{rrrrrr}
18.1 & 11.4 & 9.4 & 9.3 & 11.1 & 12.1 \\
& & & & & \\
18.1 & 13.1 & 10.2 & 8.5 & 9.3 & 9.6 \\
18.1 & 13.2 & 10.5 & 9.1 & 10.4 & 11.2 \\
18.1 & 14.3 & 11.5 & 10.0 & 11.2 & 12.0 \\
18.1 & 13.9 & 11.3 & 9.8 & 11.1 & 11.9 \\
18.1 & 19.7 & 15.5 & 13.6 & 15.1 & 15.7 \\
\hline
\end{tabular}

\section{B. Bound Tests}

B1. Nominal interest rate is at baseline plus one-half standard deviations

B2. Real GDP growth is at baseline minus one-half standard deviations

B3. Non-interest current account is at baseline minus one-half standard deviation

B4. Combination of B1-B4 using 1/4 standard deviation shocks

B5. One time 30 percent nominal depreciation in 2007

1/ Defined as non-interest current account deficit, plus interest and amortization on medium- and long-term debt, plus short-term debt at end of previous period.

2/ Gross external financing under the stress-test scenarios is derived by assuming the same ratio of short-term to total debt as in the baseline scenario and the same average maturity on medium- and long term debt. Interest expenditures are derived by applying the respective interest rate to the previous period debt stock under each alternative scenario.

3/ The key variables include real GDP growth; nominal interest rate; dollar deflator growth; and both non-interest current account and non-debt inflows in percent of GDP. 


\section{FUND RELATIONS}

(As of January 31, 2006)

I. Membership Status: Joined March 11, 1946; Article VIII

\section{FinANCIAL RELATIONS}

II. General Resources Account:

$$
\begin{array}{rr}
\begin{array}{rr}
\text { In millions } \\
\text { of SDRs }
\end{array} & \begin{array}{r}
\text { In percent } \\
\text { of Quota }
\end{array} \\
306.50 & 100.0 \\
1,915.77 & 625.05 \\
0.0 & 0.0
\end{array}
$$

Quota

Fund holdings of currency

Reserve position

III. SDR Department:

In millions

of SDRs

Percent of Allocation

Net cumulative allocation

49.98

100.0

Holdings

24.30

48.62

IV. Outstanding Purchases and Loans:

In millions of SDRs

In percent of quota

Stand-By Arrangements

$1,609.26$

525.04

V. Financial Arrangements:

Type

$\begin{array}{rrrr}\begin{array}{r}\text { Approval } \\ \text { Date }\end{array} & \begin{array}{r}\text { Expiration } \\ \text { Date }\end{array} & \begin{array}{r}\text { SDR Millions } \\ \text { Approved }\end{array} & \begin{array}{r}\text { Amount } \\ \text { Drawn }\end{array} \\ & & & \\ 6 / 08 / 05 & 6 / 08 / 08 & 766.25 & 91.95 \\ 4 / 01 / 02 & 3 / 31 / 05 & 1,988.50 & 1,988.50 \\ 6 / 25 / 02 & 8 / 08 / 02 & 128.70 & 128.70 \\ 5 / 31 / 00 & 3 / 31 / 02 & 150.00 & 150.00\end{array}$


VI. Projected Payments to Fund (Obligation basis): ${ }^{1}$ (SDR millions; based on existing use of resources and present holdings of SDRs):

Forthcoming

\begin{tabular}{lllll}
\hline 2006 & 2007 & 2008 & 2009 & 2010 \\
\hline
\end{tabular}

Principal

$\begin{array}{lllll}434.33 & 540.86 & 332.27 & 246.02 & 51.96\end{array}$

Charges/interest

\begin{tabular}{lllll}
86.04 & 49.91 & 24.73 & 10.89 & 2.37 \\
\hline 520.37 & 590.77 & 35.00 & 256.91 & 54.32
\end{tabular}

Total $\begin{array}{lrrrr}520.37 & 590.77 & 357.00 & 256.91 & 54.32\end{array}$

Projected Payments to Fund (Expectation basis): (SDR millions; based on existing use of resources and present holdings of SDRs):

\begin{tabular}{llll}
\multicolumn{5}{c}{ Forthcoming } \\
\hline $2006 \quad 2007 \quad 2008 \quad 2009 \quad 2010$ \\
\hline
\end{tabular}

Principal

$\begin{array}{lllll}434.33 & 873.13 & 246.02 & 51.96 & 3.83\end{array}$

Charges/interest

\begin{tabular}{rrrrr}
$\mathbf{8 6 . 0 4}$ & 43.51 & 11.14 & 2.36 & 0.89 \\
\hline 520.37 & 916.64 & 257.16 & 54.32 & 4.72
\end{tabular}

Total

\section{NONFINANCIAL RELATIONS}

VII. Safeguards Assessment: The authorities have already implemented many of the recommendations from the safeguards assessment conducted under the current SBA, completed in September 2005. In particular, (i) Uruguay's total obligations to the Fund are now included in the BCU's financial statements; (ii) data submitted to the Fund is periodically reviewed for consistency with the Technical Memorandum of Understanding; and (iii) the criteria for the selection and appointment of the BCU's external audit firm have been amended in line with the safeguards recommendation

VIII. Exchange Rate Arrangement: The currency is the Uruguayan peso (Ur\$). Uruguay has followed an independently floating exchange rate regime since July 29, 2002. On March 13, 2005, buying and selling interbank rates for the U.S. dollar, the intervention currency, were Ur $\$ 24.25$ and Ur $\$ 24.30$, respectively. Uruguay's exchange system is free of restrictions on payments and transfers for current international transactions.

\footnotetext{
${ }^{1}$ Except for 2006, this schedule is not the currently applicable schedule of payments to the IMF. Rather, the schedule presents all payments to the IMF under the illustrative assumption that repayment expectations would be extended to their respective obligation dates by the IMF Executive Board upon request of the debtor country.
} 
IX. Article IV Consultation: The 2003 Article IV consultation was concluded by the Executive Board on July 11 (Country Report No. 03/247). Uruguay is on the standard consultation cycle governed by the provisions approved by the Executive Board on July 15, 2002. The next Article IV discussions will be held in June 2006, together with the FSAP (see below).

X. Ex Post Assessment: The Ex Post Assessment of Longer-Term Program Engagement was considered by the Executive Board on March 18, 2005 (Country Report 05/202).

XI. FSAP participation and ROSCs: The FSAP exercise was conducted over two missions (October 2005 and January 2006), completing an earlier exercise that was started in November 2001, but was subsequently suspended on account of the financial crisis in 2002. The ROSC-module on fiscal transparency was published on March 5, 2001. A ROSC-module on data dissemination practices was published on October 18, 2001.

XII. Technical Assistance: Technical assistance on tax, customs, and social security administration was provided by FAD in March 2006, June 2005, and July 2003, on tax policy in October 2005 and May 2003, and on public financial management in March 2005. In April 2003, STA provided technical assistance on adequate recording of loans funded by the FSBS. MFD has been providing substantial and continuous technical assistance since 2002 in the resolution of intervened banks, the restructuring of the public bank BROU, and recently on BHU, and in July 2005 jointly with ICM on debt management.

XIII. Resident Representative: Mr. Gaston Gelos. 


\section{RELATIONS WITH THE WORLD BANK GROUP}

(As of March 2, 2006)

The World Bank Board of Executive Directors approved a new Country Assistance Strategy (CAS) on June 9, 2005. The CAS envisages a base case scenario of up to US\$800 million in new lending over the period FY05-10. The lending program will be modulated on the strength of the Government's program and its ability to implement it, as well as the evolution in the country's creditworthiness.

The Bank's strategy in the new CAS is anchored around a series of programmatic development loans that are expected to be multi-sectoral in focus and support the Government in key policy areas including public sector management, financial sector reform, and reform of social programs. The CAS also proposes to rebuild the investment portfolio with new operations planned to support priority investments in infrastructure, social programs and innovation. Two investment operations for Integrated Natural Resources and Transport Infrastructure and Rural Access, in the amount of US\$30 million and US\$70 million respectively were approved by the Board on June 9, 2005 together with the CAS.

The previous Country CAS was approved on May 5, 2000 and a CAS Progress Report on July 25 , 2002. Following the 2002 crisis, the Bank increased its financial support, shifting to a high case lending scenario of US\$550 million for fiscal years 2002-04, concentrated in adjustment lending. A Structural Adjustment Loan (SAL I) and a Special Structural Adjustment Loan (SSAL I) were approved with the CAS Progress Report, totaling US $\$ 303$ million, to assist Uruguay in addressing structural weaknesses and in managing the economic crisis. On April 8, 2003, another structural adjustment package (SAL II and SSAL II) was approved totaling US\$252.5 million, focusing on improving public services and human development policies. Progress in implementation of SAL I and SSAL I has been satisfactory, and the last tranches in an aggregate amount of US\$100 million (US\$50 million of SAL I and US\$50 million of SSAL I) were released in October 2004.

In the context of the new CAS, the Government has reaffirmed its commitment to the objectives of SAL II taking into account the results of the 2003 referenda on petroleum products and water. With regard to SSAL II, the objectives of the program have been achieved and, with the immediate crisis over and the beginning of a new CAS period, the outstanding second and third tranches of this operation have been cancelled at the Government's request. The social reform agenda continues to be supported by a new development policy loan (DPL1), approved by the Board of Executive Directors together with the CAS on June 9, 2005. The DPL1 in an amount of US\$75 million supports reform progress in social policies over the last two years, as well as early but important measures that the new Government has taken with respect to health, education and social protection. The DPL1 has been fully disbursed. 
The current investment portfolio comprises eight projects totaling US\$389.5 million in commitments, with an undisbursed amount of US\$158.4 million as of December 31, 2005. The performance of the investment portfolio has improved significantly in CY04 and CY05, with disbursements for investment operations aggregating to US\$43.8 million and US\$ 58.6 million respectively. This is a reflection of the substantially improved economic situation, as well closer portfolio monitoring, with portfolio performance reviews being conducted every six months.

\section{Financial Relations With the World BANK GROUP \\ (In millions of U.S. dollars) \\ Commitments \\ (Net of \\ Cancellations) \\ Disbursements Amount}

\section{IBRD Operations (as of December 31, 2005)}

Agriculture

Central Government and Administration

Telecommunication

Education

Power

Transportation

Water Supply

Total

48.5
151.5
6.0
42.0
125.0
141.0
27.0

541.0
17.4

51.5

1.6

14.3

111.7

66.7

19.4

282.6
31.1

100.0

4.4

27.7

13.3

74.3

7.6

258.4

II. IFC Operations (as of December 31, 2005)

$\begin{array}{lrrrr} & \text { Loans } & \text { Equity } & \text { Quasi } & \text { Participation } \\ \text { Held } & 18.86 & 0.21 & 10.00 & 0.0 \\ \text { Disbursed } & 18.86 & 0.19 & 10.00 & 0.0\end{array}$

III. IBRD Loan Transactions (calendar year)

\begin{tabular}{lrrrrrrrrrr}
\hline & $\mathbf{1 9 9 6}$ & $\mathbf{1 9 9 7}$ & $\mathbf{1 9 9 8}$ & $\mathbf{1 9 9 9}$ & $\mathbf{2 0 0 0}$ & $\mathbf{2 0 0 1}$ & $\mathbf{2 0 0 2}$ & $\mathbf{2 0 0 3}$ & $\mathbf{2 0 0 4}$ & $\mathbf{2 0 0 5}$ \\
\hline Disbursements & 38.7 & 50.4 & 143.9 & 66.3 & 134.2 & 64.7 & 233.5 & 97.4 & 143.8 & 134.0 \\
Repayments & 69.9 & 67.9 & 64.1 & 63.2 & 57.9 & 72.5 & 75.3 & 78.2 & 80.2 & 103.6 \\
& & & & & & & & & & \\
Net Lending & $\mathbf{- 3 1 . 2}$ & $\mathbf{- 1 7 . 5}$ & $\mathbf{7 9 . 8}$ & $\mathbf{3 . 1}$ & $\mathbf{7 6 . 3}$ & $\mathbf{- 7 . 8}$ & $\mathbf{1 5 8 . 2}$ & $\mathbf{1 9 . 2}$ & $\mathbf{6 3 . 6}$ & $\mathbf{3 0 . 4}$ \\
\hline
\end{tabular}




\section{RELATIONS WITH THE INTER-AMERICAN DEVELOPMENT BANK}

(As of February 28, 2005)

The Inter-American Development Bank (IDB) is currently preparing a new Country Strategy for Uruguay for 2005-2009. The strategy supports the newly elected Government's policy, which seeks to consolidate growth and improve the population's social welfare. The new Bank strategy will focus on the following priority areas: (i) improving public sector management, to increase its efficiency and efficacy, while supporting fiscal and debt sustainability; (ii) enhancing regional and international competitiveness of domestic output and encouraging private investment in order to promote sustainable growth; and (iii) reducing poverty and increasing social inclusion. The IDB Board of Executive Directors is expected to approve the Country Strategy in March 2006.

The Bank's new strategy proposes a likely lending scenario of about US\$950 million for the five-year period 2005-2009, which together with a normal implementation of the loan portfolio, mainly investment projects, will allow for the net lending flows to remain relatively neutral. The lending program for 2005-2006 and a tentative program for the later years - 2007-2009 — was agreed with the new authorities during the Bank's Programming Mission on August 22, 2005. This program includes lending to support the Government in the key policy areas of the social sectors, competitiveness and public sector management. To this end, a social sector loan for US\$250 million was approved on August 3, 2005, to support the development and implementation of Government's social policy aimed at reducing poverty, improving the human resource base among the poor, and strengthening the sector's institutional framework. A sectoral program to improve competitiveness for US $\$ 100$ million is under preparation with approval expected in 2006. Within the public sector management area, a three-year programmatic loan, to support improvements in tax administration and public sector management, is under preparation, with expected approval in 2006; the estimated financing for the first year amounts to US\$50 million while for the next two years the amount is to be determined. The lending program also includes investment projects with new operations planned in support of transport infrastructure, sanitation in Montevideo and social programs such as a neighborhood improvement programs. During 2005 a loan for a program to support the productivity and development of new livestock products, for US\$15.8 million, was approved in July and financing in support of the debt management unit, for US\$2.5 million, was approved in November.

As of February 27, 2006 the Bank's current portfolio in Uruguay includes loans for the financing of 15 investment projects; and two sector loans, for strengthening the banking sector and for the social sectors. The lending portfolio amounts to US\$1,188.6 million, of which US\$526.4 million are pending disbursement. Portfolio performance was affected by the Government's fiscal constraint, which entailed cuts in budgetary allocations to levels below the required amounts in order to maintain a normal pace of project implementation. A 
main challenge ahead will be to increase the pace of project execution while setting the stage for a normal implementation of the new projects.

\section{FINANCIAL RELATIONS WITH THE INTER-AMERICAN DEVELOPMENT BANK}

(In millions of U.S. dollars)

Total outstanding loans: US\$1,852.9

Loan transactions:

\begin{tabular}{lrrrrrrr}
\hline & $\mathbf{1 9 9 9}$ & $\mathbf{2 0 0 0}$ & $\mathbf{2 0 0 1}$ & $\mathbf{2 0 0 2}$ & $\mathbf{2 0 0 3}$ & $\mathbf{2 0 0 4}$ & $\mathbf{2 0 0 5}^{\mathbf{2}}$ \\
\hline Disbursements & 358.5 & 162.9 & 214.2 & 558.6 & 367.2 & 53.1 & 242.3 \\
Amortization & 57.0 & 59.4 & 60.7 & 73.1 & 103.7 & 113.3 & 220.0 \\
Net Loan Flows & $\mathbf{3 0 1 . 5}$ & $\mathbf{1 0 3 . 5}$ & $\mathbf{1 5 3 . 5}$ & $\mathbf{4 8 5 . 5}$ & $\mathbf{2 6 3 . 6}$ & $\mathbf{- 6 0 . 2}$ & $\mathbf{2 2 . 3}$ \\
\hline
\end{tabular}

Source: Inter-American Development Bank.

${ }^{1}$ IDB, as of February 27, 2006

${ }^{2}$ IDB, as of December 31, 2005 
March 13, 2006

Dear Mr. de Rato:

Performance under our program remains strong, with robust growth and low inflation. Both the primary fiscal balance and the overall balance exceeded the 2005 program objectives. Improved capital market access and buoyant exports have allowed Uruguay to significantly strengthen its official reserve position. Preliminary data suggest that all the end-2005 quantitative performance criteria (PCs) were met, some with large margins. Final data will be available before the Board meeting. Our structural reform agenda is progressing well: we have adopted a plan for the restructuring of the housing bank BHU, and we will submit a comprehensive tax reform to Congress by March 20 consistent with the program. We have also been advancing in other key reforms for 2006, including in the pension, tax administration, and financial sector areas. The growth commission is about to publish its recommendations, which will be a focus of the next review.

In light of this strong performance and in support the program objectives, we request: (i) completion of the Third Review under the SBA and purchase of SDR 85.82 million; (ii) modification of the 2006 fiscal PCs (primary balance, non-interest expenditure and debt) to accommodate critical public investment financed through privatization proceeds; (iii) waiver of nonobservance of the tax reform PC (to be submitted with a slight delay in March as a prior action for this review); (iv) modification of the end-June tax reform PC to move the approval date to end-October to accommodate expected bottlenecks in the congressional agenda; and (v) establishment of two structural performance criteria in banking sector restructuring. We have updated our economic and policy targets for 2006, as described in detail in the attached supplementary Memorandum of Economic and Financial Policies. The next review under the arrangement will be completed by June 2006. It will assess overall performance under the program and observance of the associated performance criteria and benchmarks (Tables 1 and 2). Should the external position turn out significantly stronger than we currently anticipate, we will consider reducing the net use of Fund resources by treating the arrangement as precautionary in the future or making advanced repurchases.

As usual, we will maintain a close policy dialogue with the Fund and stand ready to take additional measures as appropriate to ensure the achievement of the program's objectives.

Sincerely yours,

$/ \mathrm{s} /$
Walter Cancela
President
Central Bank of Uruguay

$\frac{/ \mathrm{s} /}{\text { Danilo Astori }}$

Attachments:

Supplementary Memorandum of Economic and Financial Policies

Technical Memorandum of Understanding 


\section{Supplementary Memorandum of Economic ANd FinanCial Policies ${ }^{1}$}

\section{Recent developments}

1. Economic performance remains strong. Growth in 2005 is likely to have exceeded the 6 percent target, led by strong consumption and exports. Inflation has increased to 6.6 percent in February 2006, and it is now within the central bank's (BCU) target range for March (5-7 percent). The 2005 fiscal primary surplus reached 3.8 percent of GDP and the overall deficit fell below 1 percent of GDP, both better than programmed. All quantitative performance criteria (PCs) for end-2005 are expected to have been met, some with large margins, and the end-March 2006 PCs are well within reach. While the indicative endDecember base money target was exceeded, this was the result of temporary liquidity needs.

2. Near-term vulnerabilities have declined with a significant strengthening of the international reserve position, reflecting buoyant exports and improved capital market access. Taking advantage of appreciation of pressures on the peso, the BCU has also intervened in the foreign exchange market. Medium-term vulnerabilities, however, remain high, with the still high public debt, mainly in foreign currency and at floating rate, and large public financing needs (averaging 8 percent of GDP) over the next three years.

\section{Fiscal policies}

3. Achieving debt sustainability through continued fiscal consolidation and sustained growth remains at the core of our program. During July 2006-June 2007 we intend to undertake additional and carefully selected one-time investment outlays, to catalyze much larger private investment in key infrastructure bottlenecks and foster higher growth. This could temporarily reduce the primary surplus slightly (by up to about a $1 / 4$ percent of GDP), but would be fully financed by non-debt creating flows (i.e. privatization proceeds from the sale of NBC). The government will adequately share the risks in these projects with private partners and record any government guarantees (which are not expected to be issued) or fiscal contingencies in the fiscal accounts. Moreover, they are required to have a positive fiscal impact in the short term (through higher revenues or lower recurrent spending) and result in productivity gains for the economy and lasting infrastructure improvements. Projects identified so far include investments in the railroad system (to facilitate exports of the forestry sector) and dredging of the port of Montevideo.

4. Our 2006 fiscal program targets an increase in revenues of about one percent of GDP to ensure both appropriate spending in priority areas (including the social emergency plan) and meeting the primary surplus target. We are therefore pressing ahead with administrative reforms, expected to yield at least $1 / 2$ percent of GDP this year (see below). The remaining $1 / 2$ percent of GDP in additional revenue would come from an improvement in the current

\footnotetext{
${ }^{1}$ This memorandum supplements the Memorandum of Economic and Financial Policies (MEFP) of May 24 and its supplements of September 14 and December 27, 2005.
} 
primary surplus of public enterprises as a result of the recovery from the drought in 2005, lower transfers to the central government, and adjusting tariffs to maintain appropriate margins and reflect cost developments.

5. We will closely monitor fiscal developments, execute expenditure cautiously, and stand ready to take measures as needed to ensure meeting the 2006 program targets. We reiterate our commitment to save any overperformance toward achieving the medium-term primary surplus target of 4 percent of GDP as soon as possible.

\section{Structural fiscal reforms and debt management}

6. Tax reform. We are about to submit to congress a comprehensive tax reform (with a slight delay with respect to the end-February performance criterion). While we are now envisaging congressional approval by October, instead of June, we will work in parallel to advance the preparation of implementing regulations and procedures to ensure that the reform becomes effective in January 2007. The draft bill is broadly in line with the proposal presented to the public last year, which aims at improving the system's equity and efficiency, including by: (i) introducing a personal income tax; (ii) revamping the corporate income tax; (iii) lowering VAT rates and broadening its base; (iv) eliminating sectoral exemptions and unifying rates for employers' social security contributions; and (v) streamlining the number of taxes. The proposal is designed to be at least revenue neutral, and envisages now a more gradual VAT rate reduction so as to safeguard the fiscal targets.

7. Revenue administration. We are pressing ahead with the reforms launched in 2005, including by ensuring an adequate cadre of human resources. The social security agency (BPS) is making good progress with its action plan. Work of the customs reform task group is on schedule to have a comprehensive reform plan by August 2006.

8. Budget process. We have established a committee to steer reforms in this area. The committee is preparing a comprehensive reform plan (structural benchmark for August 2006), including the preparation of a draft organic budget law to unify the framework regulating the formulation, coverage, classification, execution, control, and transparency of the budget process.

9. Debt management. While a detailed debt strategy will be prepared by the newly established debt management unit at the MEF, we have already started to improve the debt profile, including by replacing more expensive IDB/World Bank debt coming due next year by cheaper long-term market financing.

\section{Monetary and exchange rate policies}

10. We have maintained our monetary framework by targeting preannounced one-yearahead inflation objectives within a flexible exchange rate regime, and remain committed to a $4 \frac{1}{2}-6 \frac{1}{2}$ percent inflation in 2006 . As part of our gradual move toward a full-fledged inflation regime, we recently modified our public communication strategy by replacing announcements of quarterly base money target ranges by indicative one-year M1 targets. 
Since last December's announcement of the end-2006 target for M1 growth of 12.3 percent (in line with aggregate demand), inflationary pressures may have somewhat increased. While it is too early to assess whether the balance of risks has fundamentally shifted, we have begun to reassess policies and, in the interim, we maintain the program's more conservative indicative 2006 monetary targets. We will continue to monitor monetary and price developments closely and stand ready to adjust the monetary stance to attain our inflation objective. To continue to advance toward full-fledged inflation targeting, we are requesting TA from the Fund to improve our forecasting and policy analysis models.

11. Continued foreign exchange purchases by the BCU, and a recent US\$500 million bond issue have contributed to a significant strengthening in our reserve position. While this also led temporarily to a relatively constant exchange rate with respect to the US dollar, it has since moved in line with market conditions, and consistent with our commitment to a flexible exchange rate. Nevertheless, with our reserve position still relatively low compared with other dollarized economies, we will continue to take advantage of favorable conditions to further build reserves, including through additional purchases in the foreign exchange market, while sterilizing their monetary impact. We will make sure that this remains consistent with our inflation objective.

\section{Central bank and financial sector reforms}

12. Housing bank BHU. We have adopted an action plan that envisages a fully capitalized and commercially run housing bank. Key steps include: (i) shifting nonperforming loans and their management responsibility to a fiduciary trust and adequately capitalizing the bank (new PC for end-August 2006); and (ii) transferring the remaining nonperforming assets in BHU and their management responsibility to a fiduciary trust and review the regulatory framework, and adopt changes if needed, to ensure that BHU limits its activities to residential mortgage lending on a commercial basis. We also plan to have the restructuring plan implemented by year's end, with BHU meeting all prudential limits and having a plan in place that demonstrates its ability to generate profits and a positive cash flows going forward (new PC for end-November 2006). In the interim, BHU will not resume its lending, unless the Superintendency for Banking Supervision concludes that sufficient progress has been made in implementing its restructuring that such lending does not pose a risk to the safety and soundness of the institution. BHU will continue to service the note to BROU on schedule, and if necessary, the government will honor its guarantee.

13. $\boldsymbol{C O F A C}$. Following large deposit withdrawals, this small cooperative was intervened in early 2006, but without any negative impact on the rest of the financial system. Since then, we have made significant progress, and announced that we will fully pay out insured deposits in early March. We have also retained a firm to find a possible buyer in the near future, and should a sale not materialize in the weeks ahead, we will follow the procedures foreseen by our legal and normative framework. In any case, we will not involve any government guarantees or public funds. 


\section{Improving the investment climate and competitiveness}

14. The reform agenda prepared by the tripartite growth commission is expected to be published at end-March (structural benchmark). Proposals include, inter alia, measures to increase competition, enhance corporate transparency, improve bankruptcy legislation, strengthen the working of the judiciary system, simplify the tax and investment promotion regimes, improve public infrastructure and efficiency of public enterprises, and reduce red tape and the regulatory burden. Our program already contemplates measures in several of these areas, including through the tax reform, and new competition and bankruptcy laws. In addition, and as a focus of the next review, we will incorporate additional measures to improve investment climate into the program conditionality.

\section{Program financing and safeguards}

15. With a substantial amount of the 2006 capital market financing already secured, the 2006 financing is well in hand. We have advanced repayments to the World Bank and IDB of some US\$400 million for 2006-07 and plan to continue to take advantage of market conditions to lock in the favorable terms in view of our large medium-term financing needs. We also expect significant new disbursements from the World Bank and IDB (about US\$300 million) this year, and are firmly committed to implement associated reforms to ensure timely disbursements. 
Table 1. Uruguay: Quantitative Performance Criteria and Indicative Targets for the 2005-08 Program

\begin{tabular}{|c|c|c|c|c|c|c|c|c|c|c|c|c|c|}
\hline & \multirow{2}{*}{$\frac{\text { Dec. } 31}{2004 \text { Stock }}$} & \multicolumn{4}{|c|}{ June 30} & \multicolumn{4}{|c|}{ Sept. 30} & \multicolumn{4}{|c|}{ Dec. 30} \\
\hline & & $\mathrm{PC}$ & PC (adj.) & Actual & Margin (+) & $\mathrm{PC}$ & PC (adj.) & Actual & $\overline{\text { Margin }(+)}$ & $\mathrm{PC}$ & PC (adj.) & Prel. & Margin $(+)$ \\
\hline \multicolumn{14}{|l|}{ A. Quantitative performance criteria } \\
\hline \multicolumn{14}{|c|}{ (In millions of Uruguayan pesos) } \\
\hline $\begin{array}{l}\text { 1. Combined public sector primary balance (floor) } \\
2 /\end{array}$ & $\ldots$ & 5,471 & 5,372 & 6,394 & 1,022 & 9,687 & 9,490 & 10,351 & 861 & 14,647 & 14,397 & 15,477 & 1,080 \\
\hline $\begin{array}{l}\text { 2. General government noninterest expenditure } \\
\text { (ceiling) } 2 /\end{array}$ & $\ldots$ & 23,561 & 23,539 & 22,080 & 1,459 & 34,643 & 34,665 & 32,996 & 1,670 & 46,561 & 46,683 & 45,137 & 1,546 \\
\hline 3. Net domestic assets of the BCU (ceiling) 2/ & 74,079 & 3,983 & $\begin{array}{r}3,983 \\
\text { (In m }\end{array}$ & $\begin{array}{r}-302 \\
\text { llions of }\end{array}$ & $\begin{array}{r}4,284 \\
\text { U.S. dollars) }\end{array}$ & 4,572 & 4,572 & $-16,424$ & 20,995 & $-3,910$ & $-8,416$ & $-22,449$ & 14,033 \\
\hline 4. Net international reserves of the BCU (floor) 2/ & $-2,218$ & -130 & -130 & 18 & 148 & -110 & -110 & 592 & 702 & 280 & 451 & 1,052 & 601 \\
\hline 5. Nonfinancial public sector gross debt (ceiling) $3 /$ & 12,189 & 12,510 & 12,495 & 12,229 & 266 & 12,575 & 12,566 & 12,496 & 70 & 12,550 & 12,745 & 12,650 & 95 \\
\hline $\begin{array}{l}\text { 6. Accumulation of external payment arrears of } \\
\text { the public sector (on a continuous basis) } 2 /\end{array}$ & 0 & 0 & 0 & 0 & $\mathbf{0}$ & 0 & 0 & 0 & $\mathbf{0}$ & 0 & 0 & 0 & $\mathbf{0}$ \\
\hline \multicolumn{14}{|l|}{ B. Indicative targets } \\
\hline \multicolumn{14}{|c|}{ (In millions of Uruguayan pesos) } \\
\hline 7. Combined public sector overall balance (floor) $2 /$ & $\ldots$ & $-5,086$ & $-5,186$ & $-2,794$ & 2,391 & $-7,459$ & $-7,656$ & $-4,597$ & 3,059 & $-7,072$ & $-7,322$ & $-3,269$ & 4,053 \\
\hline 8. Monetary base (ceiling) $2 /$ & 15,648 & 557 & 557 & 1,070 & -513 & 1,673 & 1,673 & 498 & 1,175 & 3,468 & 3,468 & 4,460 & -992 \\
\hline 9. Floating debt of the central government (ceiling) & 3,081 & 3,081 & 3,081 & 2,567 & 514 & 3,081 & 3,081 & 2,298 & 783 & 3,081 & 3,081 & 2,482 & 599 \\
\hline
\end{tabular}


Table 1: Quantitative Performance Criteria and Indicative Targets for the 2005-08 program (concluded)

\begin{tabular}{|c|c|c|c|c|c|c|c|c|}
\hline & \multirow{2}{*}{$\begin{array}{r}2005 \text { Stock } \\
\text { Sept. } 30\end{array}$} & \multicolumn{4}{|c|}{2006 Targets } & & \multirow{2}{*}{$\begin{array}{l}2007 \text { IT } \\
\text { Dec. } 31\end{array}$} & \multirow{2}{*}{$\begin{array}{l}2008 \text { IT } \\
\text { Dec. } 31\end{array}$} \\
\hline & & Mar. 31 & Jun. 30 & Sept. 30 & $\overline{\text { Dec. } 31}$ & & & \\
\hline \multicolumn{9}{|l|}{ A. Quantitative performance criteria } \\
\hline \multicolumn{9}{|c|}{ (In millions of Uruguayan pesos) } \\
\hline 1. Combined public sector primary balance (floor ) $2 /$ & n.a. & 2,651 & 5,196 & 10,434 & 16,473 & & 19,311 & 20,748 \\
\hline 2. General government noninterest expenditure (ceiling) 2/ & n.a. & 13,409 & 27,050 & 39,870 & 53,558 & & 57,735 & 63,095 \\
\hline 3. Net domestic assets of the BCU (ceiling) 2/3/ & 51,525 & $-3,136$ & $-4,195$ & $-8,003 \quad 5 /$ & $-7,130$ & $5 /$ & $-16,203$ & $-9,522$ \\
\hline \multicolumn{9}{|c|}{ (In millions of U.S. dollars) } \\
\hline 4. Net international reserves of the BCU (floor) $2 / 3$ / & $-1,546$ & 250 & 333 & $4145 /$ & 554 & $5 /$ & 544 & 395 \\
\hline 5. Nonfinancial public sector gross debt (ceiling) 4/ & 12,309 & 12,882 & 13,074 & 13,082 & 12,995 & & 13,150 & 13,286 \\
\hline $\begin{array}{l}\text { 6. Accumulation of external payment arrears of the public sector } \\
\text { (on a continuous basis) } 2 /\end{array}$ & 0 & 0 & 0 & 0 & 0 & & 0 & 0 \\
\hline B. Indicative targets & & & & & & & & \\
\hline \multicolumn{9}{|c|}{ (In millions of Uruguayan pesos) } \\
\hline 7. Combined public sector overall balance (floor) $2 /$ & n.a. & $-3,076$ & $-5,528$ & 6,255 & $-4,983$ & & $-2,966$ & $-2,537$ \\
\hline 8. Combined public sector primary balance (extended) (floor) 2/ & n.a. & 2,651 & 5,196 & 10,434 & 16,473 & & 19,311 & 20,748 \\
\hline 9. Monetary base (ceiling) 2/3/ & 16,146 & 3,140 & 3,261 & 2,105 & 4,882 & & 1,803 & 1,720 \\
\hline
\end{tabular}

$\mathrm{PC}=$ Performance Criterion; IT=Indicative Target.

Sources: Ministry of Economy and Finance; and Central Bank of Uruguay.

$1 /$ As defined in the Technical Memorandum of Understanding.

$2 /$ Cumulative changes from the previous calendar year.

3/ 2006 targets are cumulative from end-September 2005.

4/ All maturities.

5/ NDA and NIR targets for end-September and end-December 2006 are indicative targets. 
Table 2. Uruguay: Structural Conditionality under the 2006-08 Program

A. Prior action

Tax reform

Submit to congress a comprehensive tax reform as described in paragraph 7 of the MEFP.

\section{B. Structural performance criteria (Country Report No. 06/123)}

Fiscal Have in place a five-year spending plan, complemented with revenue projections and deficit targets consistent with the program's fiscal targets.

Tax reform

Submit to congress a comprehensive tax reform as described in paragraph 7 of the MEFP.

October 31, 2006

C. New structural performance criteria

BHU

Move nonperforming loans into a fideicomiso and adequately capitalize BHU as specified in paragraph 11 of the TMU.

BHU

Transform BHU into an institution with a viable business plan and a strong regulatory framework as specified in paragraph 12 of the TMU.

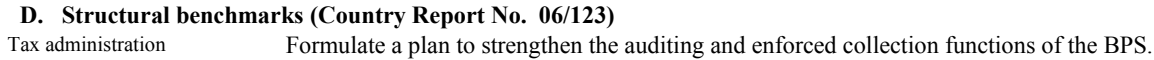
$\begin{array}{ll}\text { Tax administration } & \text { Formulate a plan to strengthen the auditing and enforced collection functions of the BPS. } \\ \text { Tax administration } & \text { Finalize the design of a comprehensive reform plan for the customs agency (including establishing collection targets consistent with the }\end{array}$

Budget $\quad \begin{aligned} & \text { Prepare recommendations, with a timetable, to improve legislation, coverage, classification, formulation, controls, and transparency of the } \\ & \text { budget process. }\end{aligned}$ program). budget process.

Growth Publish agenda of growth-enhancing reforms (including timetable for implementation) prepared by the business environment commission.

Growth Submit to Congress bankruptcy law (to include Chapter-11 type corporate restructuring). 


\section{TEChNiCAL MEMORANDUM OF Understanding}

This memorandum presents the definitions of the variables included in the performance criteria and indicative targets and some structural performance criteria for the year 2006 annexed to the Memorandum of Economic and Financial Policies.

\section{A. Quantitative Performance Criteria and Indicative Targets}

1. Cumulative floor on the overall balance of the combined public sector (indicative target). The combined public sector comprises the non-financial public sector (NFPS) and the Central Bank (BCU). The NFPS comprises the central government, ${ }^{1}$ the social security system (Banco de Previsión Social, Caja Militar, and Caja Policial), the local governments (Intendencias), the public enterprises (AFE, ANCAP, ANCO, ANP, ANTEL, INC, OSE, and UTE (including Salto Grande)). The below-the-line overall balance will be measured as the negative of the sum of: (a) the net financing of the NFPS $^{2}$ minus (b) the operating balance of the BCU.

(a) The net financing of the NFPS would include the sum of: (i) increase in net claims of the domestic financial system on the NFPS (excluding government bonds and treasury bills); (ii) the increase in the net amount of NFPS bonds and treasury bills held outside the NFPS (excluding any debt issued for the capitalization of BCU and debt issued to finance the capitalization of the deposit insurance scheme up to a limit of US\$20 million); plus (iii) external bank loans and external ${ }^{3}$ supplier credits to the NFPS; plus (iv) the net increase in liabilities arising from the forward sale of NFPS assets; plus (v) the increase in net non-bank loans; ${ }^{4}$ minus (vi) the net increase

\footnotetext{
${ }^{1}$ This includes the funds managed directly in the ministries (Fondos de Libre Disponibilidad).
}

${ }^{2}$ Excluding any cash outlays related to government guarantees (principal plus interest) on the BHU and BROU's fiduciary trusts notes; change in net debt associated with the assumption of financial liabilities of BHU and its recapitalization as part of its restructuring, and any cash inflows related to loan recoveries and sale of assets which were transferred from BHU to the government as the beneficiary in the restructuring; and the change in the net debt associated with the final outcome of the litigation/arbitration with the former shareholders of Banco Comercial.

${ }^{3}$ Based on the residency principle.

${ }^{4}$ Nonbank loans are defined in accordance with point No. 9 (a) (i) of the Fund's Guidelines on Performance Criteria with Respect to Foreign Debt (Decision No. 6230-(79/140, August 3, 1979), as amended in 2000, but exclude those instruments listed in paragraph 1 (a) (i), (ii), (iii) and (iv) of this memorandum, and cover loans of the central government and public enterprises only. 
of NFPS financial assets held by the NFPS outside the domestic financial system (including the loan component of the deposit insurance scheme); plus (vii) gross revenues from the sale of public assets (net of associated fees and commissions); plus (viii) all upfront payments related to future concessions (including the sale of mobile phone licenses); plus (ix) recoveries of financial assistance provided to the banking system.

(b) The operating balance of the BCU is defined as interest earnings on gross international reserves, as defined below, and other earnings including those on other foreign and domestic assets minus operating expenses, commissions paid, and interest paid on domestic and foreign debt administered by the BCU.

- $\quad$ The floor on the overall balance of the combined public sector will be adjusted downward for capital expenditure on identified projects in the period July 2006-June $2007 .^{5}$ The downward adjustment will be limited to the amount of gross revenues (net of any associated fees and commissions) from the sale of NBC (up to a limit of Ur\$1,200 million)

2. Cumulative floor on the primary balance of the combined public sector. The combined public sector primary balance will be calculated as the overall balance measured from below the line plus interest payments. Interest payments are defined to exclude commissions and fees.

- $\quad$ The floor on the primary balance of the combined public sector will be adjusted downward (higher deficit) for capital expenditure on identified projects in the period July 2006-June 2007.The downward adjustment will be limited to the amount of gross revenues (net of any associated fees and commissions) from the sale of NBC (up to a limit of Ur\$1,200 million).

\section{Cumulative floor on the extended primary balance of the combined public} sector. The combined public sector extended primary balance will be calculated as the primary balance of the combined public sector defined in paragraph 2 minus the change in the stock of floating debt of the central government. Floating debt of the central government is defined as expenditures from: the general treasury account ("Rentas Generales"), "Rentas de Afectación Específica", payment obligations with domestic suppliers for which external and domestic debt has been earmarked, investment funds of the Ministry of Transportation and Public Works, and own funds from agencies outside the budget (Casinos). It includes debt registered in the integrated information system (SIIF), and that has been authorized for execution and confirmed by the respective central accountants, and for which no checks for payment have yet been issued. Excluded are obligations for utilities, obligations related to

\footnotetext{
${ }^{5}$ Already identified projects include railway construction and dredging in the port of Montevideo. Further projects will be identified over the next few months and the TMU amended at the time of the next review to incorporate them.
} 
subsidies (except for the forestry fund), judiciary debt and obligations falling under "Inciso 24 " of the accounting plan of the "Contaduria General de la Nacion" (excluding debt with international organizations), domestic suppliers' credits of the Ministry of Housing, and overdue payments for wages and salaries (including all related labor benefits) of public sector contractual and permanent employees, provided such overdue payments do not exceed 10 days following the date on which payments are due.

- $\quad$ The floor on the extended primary balance of the public sector will be adjusted downward for capital expenditure on identified projects in the period July 2006-June 2007. The downward adjustment will be limited to the amount of gross revenues (net of any associated fees and commissions) from the sale of NBC (up to a limit of Ur\$1,200 million).

4. Cumulative ceiling on general government expenditure applies to total (current and capital) non-interest expenditure of the central administration and the social security system (BPS) as recorded in the accounting system at the time that checks are delivered, a bank transfer is made, or the BCU notifies the receipt of a payment order. ${ }^{6}$ The non-interest expenditure of BPS excludes benefit payments. The non-interest expenditure of the central administration includes Fondos de Libre Disponibilidad but excludes transfers to the social security system, automatic transfers to the private pension funds (AFAPs), and earmarked revenue as of December 31, 2004.

- $\quad$ The ceiling on general government expenditure will be adjusted downward for any expenses arising from pension adjustments, which exceed the increase in the legal minimum adjustment.

- $\quad$ The ceiling on general government expenditure will be adjusted upward for capital expenditure on identified projects in the period July 2006-June 2007, if those expenditure fall under this ceiling. The upward adjustment will be limited to the amount of gross revenues (net of any associated fees and commissions) from the sale of NBC (up to a limit of Ur\$1,200 million).

5. Cumulative ceiling on the monetary base (indicative target). Money base is defined as the sum of (i) currency issue; (ii) nonremunerated and remunerated peso sight deposits of BROU, BHU, private banks, and other institutions defined below at the BCU; and (iii) call peso deposits of BROU, BHU, private banks, and other institutions at the BCU. Other institutions include pension funds (AFAPs), local governments, public enterprises, trust funds of the liquidated banks (FRPB), investment funds, offshore institutions (IFEs), insurance companies, exchange houses, stock brokers, and the nonfinancial private sector. The monetary base excludes central government and BPS peso deposits held at BROU subject to a 100 percent reserve requirement. The indicative target is defined as the cumulative change calculated using the monthly averages relative to the base month average.

${ }^{6}$ Expenditures by local governments are not included. 
6. Cumulative floor on the net international reserves (NIR) of the BCU. NIR is defined as the difference between the gross international reserves and BCU reserve liabilities. Gross international reserves include all foreign exchange assets that are claims on nonresidents and that are in the direct effective control of the BCU and are readily available for such purposes of the BCU as intervention or direct financing of payment imbalances. Such assets may be in any of the following forms, provided that they meet the test of effective control and ready availability for use: currency, bank deposits in nonresident institutions and government securities and other bonds and notes issued by nonresidents (with a rating not below "A" in the classification of Fitch and IBCA and Standard and Poor's or "A2" in the classification of Moody's). In addition, holdings of SDRs or of monetary gold would be included under gross international reserves (provided they meet the test of effective control and ready availability of use) as would the reserve position in the IMF, and liquid foreign assets set aside for the use of the government.

(a) Excluded from gross international reserves are all foreign currency claims arising from off-balance sheet transactions (such as derivatives instruments), capital subscriptions to international financial institutions, any assets in nonconvertible currencies, claims on any nonresident Uruguay-owned institutions, or any amounts (in all components of assets, including gold) that have been pledged in a direct or contingent way.

(b) BCU reserve liabilities include (i) all foreign currency-denominated liabilities of the BCU with original maturity of one year or less to residents and nonresidents (excluding government deposits); (ii) all certificates of deposit used to constitute reserve requirements against bank deposits; (iii) the total use of Fund credit by Uruguay; and (iv) any net position on foreign exchange derivatives vis-à-vis the peso with either residents or nonresidents undertaken directly by the BCU or by other financial institutions on behalf of the BCU, as measured in items II.2 and III.4 of the IMF's Data Template on International Reserves and Foreign Currency Liquidity.

(c) For the purpose of the NIR calculation, (i) the gold holdings of the BCU will be valued at the accounting rate of US\$42 per troy ounce; (ii) liabilities to the IMF will be valued at the rate of US\$1.44946 per SDR; (iii) gains or losses from gold swaps and other operations will be excluded; and (iv) non-U.S. dollar denominated foreign assets and liabilities will be converted into U.S. dollars at the market exchange rates of the respective currencies as of September 30, 2005.

- $\quad$ The NIR floor will be adjusted upward (downward) by any excess (shortfalls in) net financing of the NFPS defined as the difference between the stock of debt of the NFPS, as defined in para. 8 but excluding central government foreign currency deposits in domestic commercial banks and excluding any liabilities to the Fund, and schedule A below, and which is not explained by the adjustments specified in para. 8, bullet 1 (i-ix). The downward adjustment of the NIR floor will be limited to US\$400 million. 


\begin{tabular}{|lccccc|}
\hline \multicolumn{4}{c|}{$\begin{array}{c}\text { Schedule A } \\
\text { (In millions of U.S. dollars) }\end{array}$} & & \\
\hline & Sept.-05 & Mar.-06 & Jun.-06 & Sept. 06 & Dec.-06 \\
\hline $\begin{array}{l}\text { Stock of debt of the NFPS, } \\
\text { excl. CG FX deposits in } \\
\text { domestic commercial banks and } \\
\text { Fund liabilities }\end{array}$ & 10,136 & 10,530 & 10,706 & 10,828 & 10,884 \\
\hline
\end{tabular}

7. Cumulative ceiling on net domestic assets (NDA) of the BCU. NDA is defined as the difference between end-of-period monetary base and net international reserves (NIR) of the BCU as defined in paragraphs 5 and 6 . The flow of NIR will be valued at the accounting exchange rate of Ur\$23.9 per US\$1.

- $\quad$ The NDA ceiling will be adjusted downward (upward) by the Ur\$ equivalent of any upward (downward) adjustment in the NIR target of the BCU.

8. The overall nonfinancial public sector debt ceiling refers to the outstanding stock of external debt ${ }^{7}$ and domestic debt ${ }^{8}$ measured on a gross basis ${ }^{9}$ in domestic and foreign currency owed or guaranteed by the NFPS, valued in U.S. dollars at the accounting rates of Ur\$23.9 per U.S. dollar, U.S. dollar 1.2026 per Euro, Yen113.46 per U.S. dollar, U.S. dollar 1.44946 per SDR, and Ur\$1.4882 per UI. If the Ur\$ to UI rate exceeds 1.5916 the UI debt will be valued at Ur\$ 1.4882 plus the difference between the actual rate and Ur\$1.5916. The debt ceiling will exclude (i) the government guaranteed note of BHU to BROU (with

\footnotetext{
${ }^{7}$ For the definition of external debt the term "debt" has the meaning set forth in point No. 9 of the Fund's Guidelines on Performance Criteria with Respect to Foreign Debt (Decision No. 6230-(79/140, August 3, 1979), as amended in 2000), and the term "external" is defined on the residency principle. The suppliers' contracts of ANTEL with equipment providers Ericsson and NEC are expensed under goods and services as rental outlays and, therefore, excluded from the definition of nonfinancial public sector gross debt for program purposes.

${ }^{8}$ Domestic debt covers net claims of the domestic financial system on the NFPS; NFPS bonds and treasury bills held outside the NFPS and the domestic financial system; non-bank loans as defined in footnote 4; and lease arrangements of the central government and public enterprises, as defined in point No. 9 (a) (iii) of the Fund's Guidelines on Performance Criteria with Respect to Foreign Debt (Decision No. 6230-(79/140, August 3, 1979), as amended in 2000 .

${ }^{9}$ Debt outstanding with the domestic financial system (excluding BCU) will be measured net of gross deposits.
} 
outstanding obligations estimated at US\$500.8 million at end-September 2005) and the government guaranteed notes issued by the fiduciary trusts associated with the transfer of BROU's nonperforming loans (with outstanding obligations estimated at US\$400 million at end-September 2005); and (ii) net debt of the NFPS with the BCU. It will include the total stock of Fund credit outstanding to Uruguay.

- $\quad$ The NFPS debt ceiling will be adjusted (i) upward (downward) by revisions made to the actual nonfinancial public sector gross debt stock at end-September 2005; (ii) upward by a maximum of US\$500 million for the amount of debt issued to recapitalize the BCU; (iii) upward by a maximum of US\$133 million (downward) for the cumulative reduction (increase) in the net credit position of public enterprises with the BCU; (iv) upward by a maximum of US\$150 million for the costs associated with the final outcome of the arbitration/litigation to the former shareholders of Banco Comercial; (v) upward by a maximum of US\$82 million for debt issued to finance below-the-line operations of public enterprises (such as recapitalization of ANCAP's subsidiary abroad); (vi) upward by BHU restructuring costs related to the assumption of financial liabilities or capitalization up to a limit of US\$1 billion; (vii) upward by a maximum of US\$40 million for the amount of debt issued to onlend to the deposit insurance scheme; (viii) for capital expenditure on identified projects in the period July 2006-June 2007, up to a limit of US\$50 million; (ix) downward by the amount of gross revenues (net of any associated fees and commissions) (excluding receipts in form of shares) from the sale of NBC; and (x) upward (downward) by the amount that the unadjusted NIR floor is exceeded (falls short) with the upward adjustment limited to the amount of the upward revision of the NIR target, up to a limit of US\$500 million;

9. External payments arrears of the public sector are defined on the residency principle and relate to public sector debt with foreign creditors. This performance criterion is monitored on a continuous basis.

\section{B. Structural Performance Criteria}

\section{Continuous performance criterion on the timely service of bank restructuring} debt to BROU in accordance with the current payment schedule. The bank restructuring debt to BROU includes the notes to BROU guaranteed by the government and issued by the $\mathrm{BHU}$ and the fiduciary trusts that own and manage the nonperforming loans formerly on the books of BROU. Timely service is defined as the payment of agreed principal and interest by the BHU, the trusts, or the government in accordance with the timetable (and no later than the $10^{\text {th }}$ of the subsequent month) and terms presented in Schedule B. 
Schedule B. Scheduled Service of Bank Restructuring Debt to BROU (In millions of U.S. dollars)

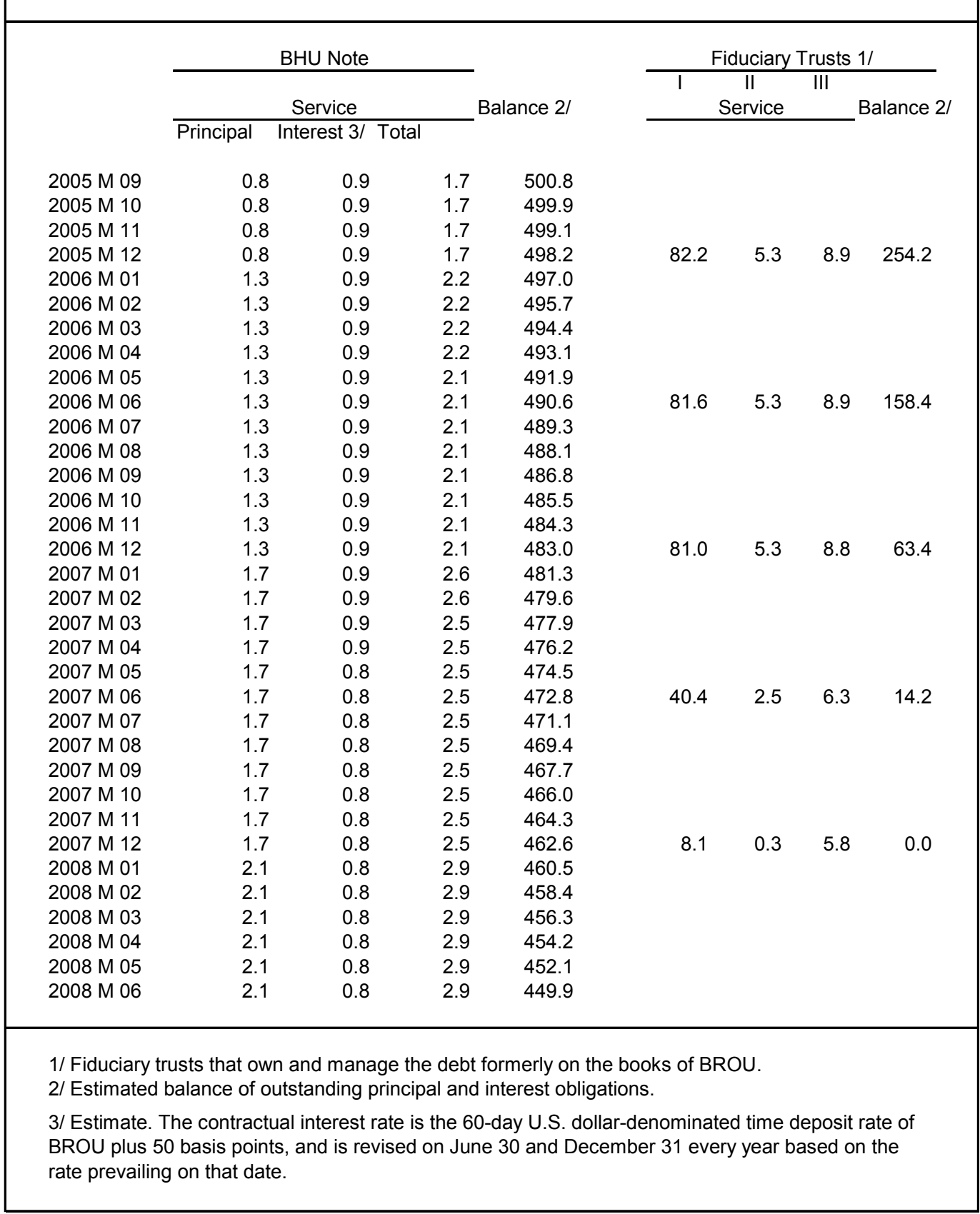


11. Restructuring and Recapitalization of BHU. The government will transfer ownership and management responsibility of nonperforming loans ${ }^{10}$ in BHU to a fideicomiso (fiduciary trust). ${ }^{11}$ The MEF will assume sufficient liabilities from BHU to recapitalize it to a level satisfactory to the Superintendency of Banks (August 31, 2006).

\section{Transform BHU into an institution with a viable business plan and a strong} regulatory framework. The government will transfer ownership and management responsibility of remaining problem assets ${ }^{12}$ in BHU to a fideicomiso. ${ }^{11}$ The government will review the regulatory framework, and modify it if needed, to ensure that BHU's business is limited to residential mortgage lending on a commercial basis. BHU will meet all prudential limits and adapt a business plan satisfactory to the Bank Superintendency that demonstrates BHU's ability to generate positive cash flows and profits, and maintain prudential capital and meet prudential regulations going forward (November 30, 2006).

\footnotetext{
${ }^{10}$ Nonperforming loans are defined to include those that are 90 days or more past due (except those that have been serviced over the last 12 months). Loans that have been serviced over the last five months could remain in BHU, but would be transferred to the fideicomiso if there are any interruptions in service.

11 The MEF will be the grantor and beneficiary of the fideicomiso. The trustee (different from $\mathrm{BHU}$ ) will be given the mandate and incentives to maximize the net present value of fideicomiso assets.

${ }^{12}$ Remaining problem assets are defined to include real estate associated with all nonperforming promitentes compradores and those that are performing but that have title problems.
} 


\section{Statement by IMF Staff Representative \\ March 24, 2006}

Since the issuance of the staff report, the following additional information has become available. It does not alter the thrust of the staff appraisal.

1. Prior action. The tax reform was submitted to congress on March 16, 2006, and is in line with the earlier draft discussed in the staff report.

2. Performance Criteria. Final data confirm that all end-December 2005 quantitative performance criteria have been observed (Table 1), with the primary surplus of the combined public sector now amounting to 3.9 percent of GDP.

3. Economic growth. Preliminary figures just released show real GDP growth in 2005 at 6.6 percent, which was led by strong exports and private consumption. This outcome is well above the 6 percent growth rate envisaged in the program.

4. Market borrowing. On March 16, Uruguay issued another US\$500 million bond, completing 80 percent of the 2006 external market financing program. This 30 -year bond was issued at a yield to maturity of 7.6 percent, with twice the duration and a lower spread than the January 24 one.

5. Resolution of the COFAC credit cooperative. Reforms have progressed as envisaged. The payout of insured deposits is being completed, and an agreement was signed for the Venezuelan Development Bank Bandes to purchase US\$10 million of COFAC's assets and liabilities to establish a new well-capitalized financial institution. 
Table 1. Uruguay: Quantitative Performance Criteria and Indicative Targets for the 2005 Program 1/

\begin{tabular}{|c|c|c|c|c|c|c|c|c|c|c|c|c|c|}
\hline & \multirow{2}{*}{$\frac{\text { Dec. } 31}{2004 \text { Stock }}$} & \multicolumn{4}{|c|}{ June 30} & \multicolumn{4}{|c|}{ Sept. 30} & \multicolumn{4}{|c|}{ Dec. 30} \\
\hline & & $\mathrm{PC}$ & PC (adj.) & Actual & Margin (+) & $\mathrm{PC}$ & PC (adj.) & Actual & Margin (+) & $\mathrm{PC}$ & PC (adj.) & Prel. & $\overline{\text { Margin }(+)}$ \\
\hline \multicolumn{14}{|l|}{ A. Quantitative performance criteria } \\
\hline \multicolumn{14}{|c|}{ (In millions of Uruguayan pesos) } \\
\hline 1. Combined public sector primary balance (floor ) $2 /$ & $\ldots$ & 5,471 & 5,372 & 6,438 & 1,066 & 9,687 & 9,490 & 10,566 & 1,076 & 14,647 & 14,397 & 15,960 & 1,563 \\
\hline $\begin{array}{l}\text { 2. General government noninterest expenditure } \\
\text { (ceiling) } 2 /\end{array}$ & $\ldots$ & 23,561 & 23,539 & 22,139 & 1,400 & 34,643 & 34,665 & 33,006 & 1,659 & 46,561 & 46,683 & 45,120 & 1,563 \\
\hline 3. Net domestic assets of the BCU (ceiling) 2/ & 74,079 & 3,983 & $\begin{array}{c}3,983 \\
\text { (In milli }\end{array}$ & $\begin{array}{r}-302 \\
\text { ons of U.S }\end{array}$ & $\begin{array}{r}4,284 \\
\text { S. dollars) }\end{array}$ & 4,572 & 4,572 & $-16,424$ & 20,995 & $-3,910$ & $-9,002$ & $-22,449$ & 13,447 \\
\hline 4. Net international reserves of the BCU (floor) 2/ & $-2,218$ & -130 & -130 & 18 & 148 & -110 & -110 & 592 & 702 & 280 & 473 & 1,052 & 578 \\
\hline 5. Nonfinancial public sector gross debt (ceiling) 3/ & 12,189 & 12,510 & 12,495 & 12,243 & 251 & 12,575 & 12,565 & 12,495 & 70 & 12,550 & 12,766 & 12,675 & 91 \\
\hline $\begin{array}{l}\text { 6. Accumulation of external payment arrears of } \\
\text { the public sector (on a continuous basis) } 2 /\end{array}$ & 0 & 0 & 0 & 0 & $\mathbf{0}$ & 0 & 0 & 0 & $\mathbf{0}$ & 0 & 0 & 0 & $\mathbf{0}$ \\
\hline \multicolumn{14}{|l|}{ B. Indicative targets } \\
\hline \multicolumn{14}{|c|}{ (In millions of Uruguayan pesos) } \\
\hline 7. Combined public sector overall balance (floor) $2 /$ & $\ldots$ & $-5,086$ & $-5,186$ & $-2,751$ & 2,435 & $-7,459$ & $-7,656$ & $-4,382$ & 3,274 & $-7,072$ & $-7,322$ & $-2,793$ & 4,529 \\
\hline 8. Monetary base (ceiling) $2 /$ & 15,648 & 557 & 557 & 1,070 & -513 & 1,673 & 1,673 & 498 & 1,175 & 3,468 & 3,468 & 4,460 & -992 \\
\hline 9. Floating debt of the central government (ceiling) & 3,081 & 3,081 & 3,081 & 2,567 & 514 & 3,081 & 3,081 & 2,298 & 783 & 3,081 & 3,081 & 2,482 & 599 \\
\hline
\end{tabular}

$\mathrm{PC}=$ performance criterion; $\mathrm{IT}=$ Indicative target.

Sources: Ministry of Economy and Finance; and Central Bank of Uruguay.

1/ As defined in the Technical Memorandum of Understanding of September 14, 2005.

2/ Cumulative changes from end-December of the previous calendar year.

3/ All maturities. 


\section{IMF Executive Board Completes Third Review Under Uruguay's Stand-By Arrangement and Approves US\$123.6 Million Disbursement}

The Executive Board of the International Monetary Fund (IMF) completed today the third review under the three-year, SDR 766.3 million (US\$1.1 billion) Stand-By Arrangement for Uruguay (see Press Release No. 05/136). Completion of this review makes an additional SDR 85.8 million (about US\$123.6 million) immediately available to Uruguay.

In completing the review, the Board granted a waiver for the non-observance of the performance criterion on the submission to Congress of a comprehensive tax reform bill, in view of the brief delay in submission.

In commenting on the Board review, Mr. Agustín Carstens, Deputy Managing Director and Acting Chair, said:

"Uruguay's performance under the Stand-By Arrangement continues to exceed expectations. Strong economic policies and a supportive external environment have contributed to rapid growth, low inflation, a strengthened external position, and an improved debt structure, and the outlook is for continued strong performance in 2006. Nevertheless, macroeconomic vulnerabilities remain and will require continued momentum in policy reform.

"The overperformance on fiscal policy is particularly welcomed, as it demonstrates the authorities'commitment to prudent fiscal policy. The ongoing efforts to strengthen tax administration and the recently submitted tax reform will be important to ensure that this continues. It is intended to establish an adjustor to the program's fiscal targets of a small margin to make room for additional, high-quality infrastructure investment, which should help to promote private investment and stimulate growth.

"Monetary policy has successfully contained inflation in the low single digits. The central bank's focus on building reserves and achieving the program's inflation objective is well placed, and appropriate exchange rate flexibility should be maintained. Incipient inflationary pressures need to be monitored carefully, and it will be important that the authorities follow up on their commitment to adjust policies as necessary to safeguard their objective of further lowering inflation. The recent establishment of a debt management office is welcomed. 
"Financial sector reforms are progressing as envisaged. The recently adopted restructuring plan for the ailing housing bank BHU should help to ensure sound new mortgage lending and minimize fiscal costs, while the recently intervened cooperative COFAC is being transformed into a new, financially sound, institution. The draft financial sector legislation submitted to congress in December, once approved, should also reduce financial sector vulnerabilities and improve the policy framework of the central bank.

"Continued strong macroeconomic polices and further progress with structural reforms will lay the basis for a lasting exit from Fund financial support, increase the economy's resilience to shocks, and raise Uruguay's growth prospects. Measures to improve the investment climate and enhance productivity will be particularly important. Also, Uruguay should continue to take advantage of favorable external conditions to further improve its debt structure," Mr. Carstens said. 


\section{Statement by Hector Torres, Executive Director for Uruguay and David Vogel, Advisor to Executive Director March 27, 2006}

1. Over the last year, Uruguay's economy has evolved robustly. Prudent macroeconomic policies, together with the government's clear commitment to strictly follow the rule of law, have been sound pillars for this performance. Meanwhile, our authorities have been developing critical structural reforms. Some have already been submitted to Congress, while others will follow soon in the very near future. The government's broad agenda that comprises fiscal, monetary and financial reforms, as well as policies aimed at reducing and alleviating poverty conditions, increasing competence and further improving the rules of the game will pave the way for a higher, sustainable and more equitable growth.

2. Economic growth has exceeded the program's original projection, with significant boosting of private consumption, investment and exports. Regarding external sales, market diversification continues to show an increasing trend, and the Uruguayan authorities are working hard to negotiate new trade agreements as a way to further integrate Uruguay into the world economy. Furthermore, Uruguay is exhibiting a remarkable performance in the fiscal area, recording a primary fiscal surplus of 3.8 percent of GDP in 2005, thus exceeding the program's target. Likewise, the employment rate has increased, although it has not yet been fully reflected in the unemployment figures given the increasing number of people willing to enter into the formal labor market.

3. The inflation rate is now in line with the Central Bank of Uruguay's (BCU) target range and beyond the benign environment, financial markets are reflecting strong confidence in the country. During 2006, Uruguay placed in international markets US\$ 500 million of sixteen-year bond in January, and US\$ 500 million of thirty-year bond in March. The yields of both bonds showed a downward trend, compared to previous issuances. Since then, the country has closed the 2006 financial gap and prefinanced some portion of 2007, having made advance payments (some of which were due in 2007) to the World Bank and InterAmerican Development Bank.

4. The authorities have already submitted a comprehensive tax reform to Congress, which will constitute a major change for the country. This reform, which will be effective by 2007, aims to improve equity and the efficiency of the tax system and to be consistent with the authorities' objective to attract productive investment. It is important to note that the government will proceed very carefully in order to avoid any revenue losses. Looking forward, and together with the authorities' goals in the expenditure area and the more efficient revenue administration, the tax reform will be critical to reduce Uruguay's high tax burden, which has historically posed a significant obstacle for private investment.

5. Regarding revenue collection, the tax administration agency (DGI) continues to display a very good performance, while the social security (BPS) and the custom administration agencies (where comprehensive plan reforms are expected this year) are attaining significant increases in their collections. The authorities are making substantial 
efforts to reduce evasion levels, and beyond simplifying the tax system, and strengthening revenue administrations, as well as increasing audits and establishing the corresponding penalties for detected non-compliance, they are formulating educational campaigns -with effect more in the medium-term- aimed at raising awareness among the population particularly among children from an early age- on the importance of paying taxes as a means to meet citizens responsibilities.

6. It is well known that citizens' ownership on expenditure composition can play an important role in the tax compliance behavior. ${ }^{1}$ In this regard, the already approved five-year budget clearly reflects the government's priorities in education, health care, and infrastructure, while showing a downward trend in public spending with little or negligible incidence on growth and social development.

7. This sound and necessary fiscal trend will lead to substantial reduction in Uruguay's debt (in 2005 debt-to-GDP fell to 70 percent from 92 the year before). At the same time, the Debt Management Office aims to further reduce the debt cost, lengthen its maturity (6 years at end-2004 to $7 \frac{1}{2}$ years at end-2005, as noted in the staff report), progressively de-dollarize (there are already some positive indications in this respect, although, clearly, much more remains to be done), as well as to substitute part of the multilateral debt, thus continuing the authorities' commitment of undertaking a sustainable and successful exit strategy from the Fund's assistance.

8. As we have stated in previous statements, the Uruguayan authorities consider that growth and a sound fiscal position are fully compatible and synergetic. Clearly, low investment rates have been the Achilles' heel of Uruguay's growth, and, in this regard, beyond establishing the needed structural reforms with the objective of boosting potential growth, the authorities consider that well-targeted public investment in infrastructure is vital to support private investment. Thus, from July 2006 to June 2007 the authorities intend to establish one-time investment outlays which may, albeit temporarily, reduce the primary surplus (up to about a $1 / 4$ percent of GDP). These outlays will be fully financed by non-debt creating flows and used to finance high-quality projects which will be carefully selected and monitored, taking into account three pre-requisites; they should comprise purely investment in infrastructure; have a positive fiscal impact by reducing from an early stage recurrent spending and/or increasing public revenues; and have a direct impact on the country's competitiveness. The government is fully committed to select these projects only after the completion of a comprehensive cost-benefit study prepared by recognized firms, as well as to ensure the minimization of contingent fiscal risks by following the best international practices in this area. As indicated in the staff report, the authorities are fully committed to pursue the primary surplus goals (saving any revenue overperformance to reach their medium-term goal as soon as possible), and that the use of this one-time adjustor should be regarded as a safety valve to allow Uruguay to undertake some critical infrastructure projects.

\footnotetext{
${ }^{1}$ For instance, among other studies, James Alm, Betty Jackson, and Michael McKee ("Fiscal exchange, collective decision institutions, and tax compliance" in Journal of Economic Behavior and Organization 22, 1993 ) conclude that "there are strong evidence that individuals are more likely to respond positively -and so to increase their tax compliance- when faced with a public sector expenditure program that they select themselves and that they know enjoys widespread approval".
} 
9. Although the inflation rate $(y / y)$ has risen in the last two months, it is important to note that it is now in line with the Central Bank target range. The authorities would like to reiterate that the main objective of the monetary policy is to keep the inflation rate low and stable. In order to meet this objective, in a context of a flexible exchange regime, the authorities will basically follow the same monetary policy, with some modifications aimed at gradually progressing towards a full-fledged inflation target regime. In this regard, the Central Bank is committed to use instruments available in a flexible way so as to meet its inflation objective.

10. It is important to note that taking advantage of the inflow of U.S. dollars, the authorities have continued to further build the level of international reserves. Admittedly, this has prevented an excessive appreciation of the peso; my authorities are fully aware that building up competitiveness goes well beyond the exchange rate; however, it is important for a country of Uruguay's characteristics and dimensions to shelter growth from the "moods of short-term financial inflows". The real effective exchange rate is in line with fundamentals and the authorities are closely monitoring the level of the nominal exchange rate in order to avoid that an eventual misalignment can pose an obstacle for production. At the same time, the authorities agree on the need to avoid signaling a floor for the exchange rate.

11. Consistent with our authorities' view that the country's competitiveness is a broad concept, they are working, as noted, to reduce the fiscal burden on production by implementing fiscal reforms, and to significantly alleviate the important cost of doing business. The consensus building effort on the government's growth agenda, whose objective is to improve the business climate, is a good example of how government, investors and workers can work together. We are confident that this effort will help put together helpful recommendations to be included in a presidential growth initiative.

12. More available and less expensive financial credit is key to spur investment, and many of the structural reforms in the financial sector will further underpin confidence in the system, thus contributing to that objective. Although it was a negative fact by itself, the recent episode of Cofac, whose problems came from the 2002 financial crisis, underlines some relevant facts: a) institutions in the financial sector (Superintendency of the Financial Sector and the newly created deposit insurance agency) have worked efficiently; b) the episode was isolated and did not have any effect on the rest of the financial sector, highlighting the eloquent progress of the banking system health, as recognized in the staff report; and c) the government's clear message that there will not be any government guarantees or public funds to bail out private creditors, which establish a sound precedent. In the case of BHU, a comprehensive restructuring plan has been adopted and its implementation will allow the country to have a sound institution, with a clear and specific mandate, good governance, and proper incentives.

13. One year after the current administration took office, with a positive performance last year, and many challenges and good perspectives ahead, the authorities would like to reiterate their gratitude to the staff for the frank dialogue held, the Management for their constant involvement, and the Executive Directors for their helpful suggestions, and for having supported Uruguay's social and economic structural reforms at a very early stage. 